\title{
MULTI-ELEMENT ABUNDANCE MEASUREMENTS FROM MEDIUM-RESOLUTION SPECTRA. II. CATALOG OF STARS IN MILKY WAY DWARF SATELLITE GALAXIES*
}

\author{
Evan N. Kirby ${ }^{1,9}$, Puragra Guhathakurta ${ }^{2}$, Joshua D. Simon ${ }^{3}$, Marla C. Geha ${ }^{4}$, Constance M. Rockosi ${ }^{2}$, \\ Christopher Sneden ${ }^{5}$, Judith G. Cohen ${ }^{1}$, Sangmo Tony Sohn ${ }^{6}$, Steven R. Majewski ${ }^{7}$, and Michael Siegel ${ }^{8}$ \\ ${ }^{1}$ California Institute of Technology, Department of Astronomy, Mail Stop 249-17, Pasadena, CA 91106, USA \\ ${ }^{2}$ University of California Observatories/Lick Observatory, Department of Astronomy \& Astrophysics, University of California, Santa Cruz, CA 95064, USA \\ ${ }^{3}$ Observatories of the Carnegie Institution of Washington, 813 Santa Barbara Street, Pasadena, CA 91101, USA \\ ${ }^{4}$ Astronomy Department, Yale University, New Haven, CT 06520, USA \\ ${ }^{5}$ McDonald Observatory, University of Texas, Austin, TX 78712, USA \\ ${ }^{6}$ Space Telescope Science Institute, 3700 San Martin Drive, Baltimore, MD 21218, USA \\ ${ }^{7}$ Department of Astronomy, University of Virginia, P.O. Box 400325, Charlottesville, VA 22904-4325, USA \\ ${ }^{8}$ Pennsylvania State University, 525 Davey Lab, State College, PA 16801, USA \\ Received 2010 March 7; accepted 2010 October 26; published 2010 November 30
}

\begin{abstract}
We present a catalog of $\mathrm{Fe}, \mathrm{Mg}, \mathrm{Si}, \mathrm{Ca}$, and Ti abundances for 2961 stars in eight dwarf satellite galaxies of the Milky Way (MW): Sculptor, Fornax, Leo I, Sextans, Leo II, Canes Venatici I, Ursa Minor, and Draco. For the purposes of validating our measurements, we also observed 445 red giants in MW globular clusters and 21 field red giants in the MW halo. The measurements are based on Keck/DEIMOS medium-resolution spectroscopy (MRS) combined with spectral synthesis. We estimate uncertainties in $[\mathrm{Fe} / \mathrm{H}]$ by quantifying the dispersion of $[\mathrm{Fe} / \mathrm{H}]$ measurements in a sample of stars in monometallic globular clusters (GCs). We estimate uncertainties in $\mathrm{Mg}, \mathrm{Si}, \mathrm{Ca}$, and $\mathrm{Ti}$ abundances by comparing to high-resolution spectroscopic abundances of the same stars. For this purpose, a sample of 132 stars with published high-resolution spectroscopy in GCs, the MW halo field, and dwarf galaxies has been observed with MRS. The standard deviations of the differences in $[\mathrm{Fe} / \mathrm{H}]$ and $\langle[\alpha / \mathrm{Fe}]\rangle$ (the average of $[\mathrm{Mg} / \mathrm{Fe}],[\mathrm{Si} / \mathrm{Fe}]$, $[\mathrm{Ca} / \mathrm{Fe}]$, and $[\mathrm{Ti} / \mathrm{Fe}])$ between the two samples is 0.15 and 0.16 , respectively. This catalog represents the largest sample of multi-element abundances in dwarf galaxies to date. The next papers in this series draw conclusions on the chemical evolution, gas dynamics, and star formation histories from the catalog presented here. The wide range of dwarf galaxy luminosity reveals the dependence of dwarf galaxy chemical evolution on galaxy stellar mass.
\end{abstract}

Key words: galaxies: abundances - galaxies: dwarf - Galaxy: evolution - Local Group

Online-only material: color figures, machine-readable tables

\section{INTRODUCTION}

The Milky Way contains $\sim 5 \times 10^{10} M_{\odot}$ of stars. Approximately $90 \%$ of those stars lie in the disk, $9 \%$ in the bulge, and $1 \%$ in the halo (Binney \& Tremaine 2008). However, these structures did not form their stars en masse. The sites of star formation are far less massive than any of these components. For example, star formation in the disk occurs in open clusters, which typically have just $300 M_{\odot}$ of stars (Piskunov et al. 2007). Dwarf galaxies, which may have built the bulge and halo (Searle \& Zinn 1978; White \& Rees 1978), range in mass from $10^{3} M_{\odot}$ (e.g., Segue 1 ; Martin et al. 2008) to more than $10^{7} M_{\odot}$ (e.g., Fornax; Irwin \& Hatzidimitriou 1995; Mateo 1998). The pockets of star formation within the dwarf galaxies likely contained just as little mass as the open clusters in the Galactic disk. Dynamical processes assemble the many disparate sites of star formation into the primary Galactic components, such as the disk, bulge, and halo. Therefore, the key to discovering the origins of stars lies in studying stellar populations in less massive structures.

The dwarf galaxies that orbit the Milky Way (MW) present the opportunity to study the formation of stellar populations most similar to the MW stellar halo. Unlike most open clusters,

\footnotetext{
* Data herein were obtained at the W. M. Keck Observatory, which is operated as a scientific partnership among the California Institute of Technology, the University of California, and NASA. The Observatory was made possible by the generous financial support of the W. M. Keck Foundation.

9 Hubble Fellow.
}

dwarf galaxies contain old, metal-poor stars. Unlike most globular clusters (GCs), dwarf galaxies enable the study of temporally extended star formation, which ultimately results in chemical evolution. Furthermore, galaxies of this class probably created at least some of the stellar halo of the MW by gravitational dissolution (e.g., Majewski 1993; Majewski et al. 1996; Bell et al. 2008). If so, then the stellar population of the present MW halo contains a mixture of many different galaxies. The individual stellar populations are difficult to disentangle, especially in the inner halo (e.g., Cooper et al. 2010). Surviving dwarf galaxies may offer a look at single, though evolved, counterparts to an accreted component of the halo. The stellar populations in surviving and accreted dwarfs differ because the accretion time is correlated with stellar mass and therefore star formation history. Nonetheless, the observed abundance differences can be compared with models of surviving galaxies and hierarchically formed halos (e.g., Robertson et al. 2005; Font et al. 2006).

Alternatively, the halo may have been created by a combination of monolithic collapse (Eggen et al. 1962) and hierarchical assembly. Comparisons of the abundances of dwarf galaxy stars and the chemodynamics of halo stars (e.g., Gratton et al. 2003; Venn et al. 2004) show that only some of the stars in the halo are consistent with accretion of dwarf galaxies similar to the surviving dwarfs. Although it is clear that the nearby halo stars have different abundance patterns than dwarf satellite galaxies, the number of dwarf galaxy stars with published multi-element abundance measurements is less than for the halo. The relative 
distance of dwarf galaxies compared to the nearest observable halo stars has prevented the sample of dwarf galaxy abundances from growing as fast as for halo stars. Studying the role of dwarf galaxies in building the halo would benefit from a larger sample of dwarf galaxy elemental abundances. (Roederer 2009 points out that even the halo abundances have not been well sampled because the more distant halo stars have not been observed nearly as completely as nearby halo stars.)

Stellar elemental abundances reveal many characteristics of the stellar population of a galaxy. Its metallicity distribution, usually represented by the stellar iron content, is a function of the galaxy's total mass, the amount of gas infall and outflow, the chemical yield of supernovae, whether the galaxy formed out of gas pre-enriched with metals, and other variables. Beyond the one-dimensional metallicity distribution, the ratios of other elements to iron reflect other details of the star formation history. In particular, the ratios of alpha elements, such as magnesium, to iron depend on the duration and intensity of star formation.

A large sample of multi-element abundances for individual stars in dwarf galaxies would be a useful tool in studying star formation in small systems. However, obtaining elemental abundances is expensive. High-quality, high-resolution spectroscopy (HRS; $R>15,000)$ allows the measurements of tens of elements to precisions of $<0.2$ dex for stars with $V \lesssim 18.5$. This magnitude limit restricts the measurement of elemental abundances to the upper 1-2 mag of the red giant branch for most MW dwarf galaxies. In addition, most current high-resolution spectrographs operate on one star at a time. The exposure time required for adequate signal to derive accurate abundance measurements has limited the sample of stars in intact MW dwarf satellite galaxies (excluding Sagittarius) with published HRS to fewer than 200.

With current technology, a statistical analysis of the elemental abundance distributions in dwarf galaxies must rely on mediumresolution spectroscopy (MRS; $R \sim 7000$ ). MRS relaxes the magnitude limit to $V \lesssim 21.5$ while permitting multiplexing of more than one hundred stars in one exposure. Building a large sample of multi-element abundances in MW dwarf galaxies requires both of these capabilities. The disadvantages of MRS are that fewer elements are accessible than with HRS, and the measurements are less precise. Tolstoy et al. (2001) presented one of the first applications of MRS to dwarf galaxy metallicity distributions. They based their metallicity measurements on a calibration of infrared calcium triplet equivalent width to [Fe/H] (e.g., Armandroff \& Da Costa 1991; Rutledge et al. 1997). However, spectral synthesis is required to measure the abundances of multiple elements. Several recent spectral synthesis-based studies have employed MRS to begin building the sample of abundances in MW dwarf galaxies. Kirby et al. (2008b) discovered extremely metal-poor $([\mathrm{Fe} / \mathrm{H}]<-3)$ stars in the ultra-faint dwarf galaxies using Keck/DEIMOS spectroscopy. Shetrone et al. (2009) measured Fe, Mg, Ca, and Ti abundances for 27 stars in the Leo II galaxy using Keck/ LRIS spectroscopy. Finally, Kirby et al. (2009, hereafter Paper I) measured $\mathrm{Fe}, \mathrm{Mg}, \mathrm{Si}, \mathrm{Ca}$, and $\mathrm{Ti}$ abundances for 388 stars in the Sculptor dwarf galaxy using Keck/DEIMOS spectroscopy.

In this article, we present a catalog of elemental abundance measurements of 2961 stars in eight MW dwarf galaxies. The technique is very similar to that developed by Kirby et al. (2008a, hereafter KGS08) and modified as noted in Paper I. We describe the observations in Section 2. In Section 3, we briefly summarize the technique and present the catalog. In Section 4, we explore the accuracy of the measurements using repeat observations and comparison to HRS measurements of the same stars. We summarize our work and discuss our articles that interpret these data in Section 5.

The next papers in this series apply the data presented here to deducing the past chemical evolution histories of the eight dwarf spheroidal galaxies (dSphs). Paper III (Kirby et al. 2010b) includes galactic chemical evolution models of the metallicity distributions. Paper IV (Kirby et al. 2010a) shows how the $\langle[\alpha / \mathrm{Fe}]\rangle$ ratios change with $[\mathrm{Fe} / \mathrm{H}]$, a diagnostic of the past intensity of star formation. Both papers examine trends with the total luminosity of the dSph. This large data set, encompassing a wide luminosity range of MW satellites, will uniquely enable an exploration of the dependence of chemical evolution on dwarf galaxy mass.

\section{OBSERVATIONS}

The medium-resolution abundances in this article depend on spectroscopy obtained with the Deep Imaging Multi-Object Spectrograph (DEIMOS; Faber et al. 2003) on the Keck II telescope and photometry from a variety of sources. This section explains how spectroscopic targets were selected from photometric catalogs and how the DEIMOS observations were performed.

Every spectroscopic target is an individual star in one of three types of stellar system: GC, the MW halo, and dwarf galaxy. All of the target systems except the MW halo field stars are listed in Table 1. The MW halo field stars are included in Table 7. The $\mathrm{GC}$ and halo field stars are interesting in their own right. The GC stars in particular will be examined in further detail in a future work. However, we examine the GC and halo field stars here only to assess the accuracy of the abundance measurement technique. The observations most relevant to this article's scientific focus are the individual dSph stellar spectra.

\subsection{Globular Clusters}

Stars in most GCs are excellent metallicity standards because every star in a given cluster has the nearly the same iron abundance and the same heliocentric distance. Exceptions include $\omega$ Cen (Freeman \& Rodgers 1975) and M22 (Da Costa et al. 2009; Marino et al. 2009). We targeted individual stars in 12 monometallic GCs, listed in Table 1 . We check stellar abundances within each cluster for internal consistency, and we also check the DEIMOS-derived abundances against HRS abundance measurements.

Stetson (2000) has made photometry publicly available for all of these clusters as part of his photometric standard field database. ${ }^{10}$ Every star has been measured in at least two of the Johnson-Cousins $B, V, R$, and $I$ filters. This database does not contain every star in each field, but only those stars suitable for photometric standardization. Therefore, P. B. Stetson (private communications, 2007, 2008) generously provided complete photometric data for our targets in M13, M71, NGC 7006, M15, and NGC 7492.

We supplemented Stetson's photometry with additional photometry for five clusters, mostly to increase the field of view so that it spanned a full DEIMOS slitmask. For M79, we utilized the $U B V$ measurements of Kravtsov et al. (1997) and the VI measurements of Rosenberg et al. (2000). For M5, we supplemented Stetson's photometry with the ugriz Sloan Digital Sky

\footnotetext{
${ }^{10}$ http://www2.cadc-ccda.hia-iha.nrc-cnrc.gc.ca/community/ STETSON/standards/
} 
Table 1

Spectroscopic Targets

\begin{tabular}{llccc}
\hline \hline \multicolumn{1}{c}{ Target } & $\begin{array}{c}\text { R.A. } \\
(\mathrm{J} 2000)\end{array}$ & $\begin{array}{c}\text { Decl. } \\
(\mathrm{J} 2000)\end{array}$ & $\begin{array}{c}\text { Distance } \\
(\mathrm{kpc})\end{array}$ & $\begin{array}{c}(\mathrm{m}-\mathrm{M})_{0}{ }^{\mathrm{a}} \\
(\mathrm{mag})\end{array}$ \\
\hline \multicolumn{5}{c}{ Globular clusters } \\
\hline NGC 288 & $00^{\mathrm{h}} 52^{\mathrm{m}} 45^{\mathrm{s}}$ & $-26^{\circ} 34^{\prime} 43^{\prime \prime}$ & 8.8 & 14.74 \\
NGC 1904 (M79) & $05^{\mathrm{h}} 24^{\mathrm{m}} 11^{\mathrm{s}}$ & $-24^{\circ} 31^{\prime} 27^{\prime \prime}$ & 12.9 & 15.56 \\
NGC 2419 & $07^{\mathrm{h}} 38^{\mathrm{m}} 09^{\mathrm{s}}$ & $+38^{\circ} 52^{\prime} 55^{\prime \prime}$ & 84.3 & 19.63 \\
NGC 5904 (M5) & $15^{\mathrm{h}} 18^{\mathrm{m}} 34^{\mathrm{s}}$ & $+02^{\circ} 04^{\prime} 58^{\prime \prime}$ & 7.5 & 14.37 \\
NGC 6205 (M13) & $16^{\mathrm{h}} 41^{\mathrm{m}} 41^{\mathrm{s}}$ & $+36^{\circ} 27^{\prime} 37^{\prime \prime}$ & 7.6 & 14.42 \\
NGC 6341 (M92) & $17^{\mathrm{h}} 17^{\mathrm{m}} 07^{\mathrm{s}}$ & $+43^{\circ} 08^{\prime} 11^{\prime \prime}$ & 8.2 & 14.58 \\
NGC 6838 (M71) & $19^{\mathrm{h}} 53^{\mathrm{m}} 46^{\mathrm{s}}$ & $+18^{\circ} 46^{\prime} 42^{\prime \prime}$ & 4.0 & 13.02 \\
NGC 7006 & $21^{\mathrm{h}} 01^{\mathrm{m}} 29^{\mathrm{s}}$ & $+16^{\circ} 11^{\prime} 14^{\prime \prime}$ & 41.4 & 18.09 \\
NGC 7078 (M15) & $21^{\mathrm{h}} 29^{\mathrm{m}} 58^{\mathrm{s}}$ & $+12^{\circ} 10^{\prime} 01^{\prime \prime}$ & 10.3 & 15.06 \\
NGC 7089 (M2) & $21^{\mathrm{h}} 33^{\mathrm{m}} 27^{\mathrm{s}}$ & $-00^{\circ} 49^{\prime} 24^{\prime \prime}$ & 11.5 & 15.30 \\
Pal 13 & $23^{\mathrm{h}} 06^{\mathrm{m}} 44^{\mathrm{s}}$ & $+12^{\circ} 46^{\prime} 19^{\prime \prime}$ & 25.8 & 17.05 \\
NGC 7492 & $23^{\mathrm{h}} 08^{\mathrm{m}} 27^{\mathrm{s}}$ & $-15^{\circ} 36^{\prime} 41^{\prime \prime}$ & 25.8 & 17.06 \\
\hline & & $\mathrm{dSphs}$ & & \\
\hline Sculptor & $01^{\mathrm{h}} 00^{\mathrm{m}} 09^{\mathrm{s}}$ & $-33^{\circ} 42^{\prime} 32^{\prime \prime}$ & 85 & 19.67 \\
Fornax & $02^{\mathrm{h}} 39^{\mathrm{m}} 59^{\mathrm{s}}$ & $-34^{\circ} 26^{\prime} 57^{\prime \prime}$ & 139 & 20.72 \\
Leo I & $10^{\mathrm{h}} 08^{\mathrm{m}} 28^{\mathrm{s}}$ & $+12^{\circ} 18^{\prime} 23^{\prime \prime}$ & 254 & 22.02 \\
Sextans & $10^{\mathrm{h}} 13^{\mathrm{m}} 03^{\mathrm{s}}$ & $-01^{\circ} 36^{\prime} 52^{\prime \prime}$ & 95 & 19.90 \\
Leo II & $11^{\mathrm{h}} 13^{\mathrm{m}} 29^{\mathrm{s}}$ & $+22^{\circ} 09^{\prime} 12^{\prime \prime}$ & 219 & 21.70 \\
Canes Venatici I & $13^{\mathrm{h}} 28^{\mathrm{m}} 04^{\mathrm{s}}$ & $+33^{\circ} 33^{\prime} 21^{\prime \prime}$ & 210 & 21.62 \\
Ursa Minor & $15^{\mathrm{h}} 09^{\mathrm{m}} 11^{\mathrm{s}}$ & $+67^{\circ} 12^{\prime} 52^{\prime \prime}$ & 69 & 19.18 \\
Draco & $17^{\mathrm{h}} 20^{\mathrm{m}} 19^{\mathrm{s}}$ & $+57^{\circ} 54^{\prime} 48^{\prime \prime}$ & 92 & 19.84 \\
\hline & & & & \\
\hline
\end{tabular}

Notes.

${ }^{a}$ Extinction-corrected distance modulus.

References. The coordinates and distance moduli for the globular clusters are given by Harris (1996, updated 2003, http://www.physics.mcmaster.ca/ $\sim$ harris/mwgc.dat). Harris relied on data from the following sources: NGC 288, Bellazzini et al. (2001); M79, Ferraro et al. (1992); NGC 2419, Harris et al. (1997); M5, Brocato et al. (1996) and Sandquist et al. (1996); M13, Paltrinieri et al. (1998); M92, Carney et al. (1992); M71, Geffert \& Maintz (2000); NGC 7006, Buonanno et al. (1991); M15, Durrell \& Harris (1993); M2, Harris (1975); Pal 13, Siegel et al. (2001); NGC 7492, Côté et al. (1991). The dSph coordinates are adopted from Mateo (1998), and the distances are adopted from the following sources: Sculptor, Pietrzyński et al. (2008); Fornax, Rizzi et al. (2007); Leo I, Bellazzini et al. (2004); Sextans, Lee et al. (2003); Leo II, Siegel et al. (2010); Canes Venatici I, Kuehn et al. (2008); Ursa Minor, Mighell \& Burke (1999); Draco, Bellazzini et al. (2002)

Survey (SDSS) crowded field photometry of An et al. (2008). For consistency with the other data in the Johnson-Cousins system, we converted SDSS ugriz to Johnson-Cousins UBVRI following the global, metallicity-independent transformations of Jordi et al. (2006). All magnitudes were corrected for extinction based on $E(B-V)$ from Harris's (1996, updated $2003^{11}$ ) catalog of GC properties. In order to arrive at his values, Harris averaged measurements of $E(B-V)$ by Reed et al. (1988), Webbink (1985), Zinn (1985), and the authors listed in Table 1.

Not all targets could be observed spectroscopically due to the limited field of view and slitmask design constraints. First, we attempted to maximize the number of target stars previously observed with HRS by placing the slitmasks in regions with a high density of HRS targets. We selected the remaining targets based on the clusters' color-magnitude diagrams (CMDs). In order of priority, we filled each slitmask with stars from the (1) upper red giant branch (RGB), (2) lower RGB, (3) red clump, and (4) blue horizontal branch. For the GCs with angular sizes smaller than the DEIMOS field of view, we filled the slits at the edges of the slitmask far from the center of the GCs with

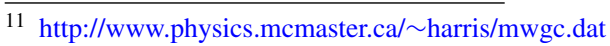

objects having similar colors and magnitudes to stars on the RGB. Simon \& Geha (2007) prioritized targets for the n1904 and 2419 slitmasks (see Table 2 ) differently. We refer the reader to their article for further information.

\subsection{Halo Field Stars}

The most metal-poor MW GC known is M15 $([\mathrm{Fe} / \mathrm{H}]=$ -2.38; Sneden et al. 1997, 2000; Pritzl et al. 2005). Dwarf galaxies contain even more metal-poor stars (e.g., Shetrone et al. 2001; Fulbright et al. 2004). In order to verify that the abundance measurements based on MRS are accurate at these very low metallicities, we obtained DEIMOS spectra of metalpoor MW halo field stars having HRS data.

We chose several studies of metal-poor halo field stars: the Keck High-Resolution Echelle Spectrograph (HIRES) and Lick/Hamilton spectrograph measurements of Johnson (2002) at $R \sim 45,000-60,000$; the Keck/HIRES measurements of Fulbright (2000), Cohen et al. (2002), and Carretta et al. (2002); the Kitt Peak National Observatory Coudé spectrograph measurements of Pilachowski et al. (1996); and the Keck Echellette Spectrograph and Imager (ESI) measurements of Lai et al. (2004, 2007) at $R \sim 7000$. Each of these authors selected targets from low-resolution surveys for metal-poor stars in the Galactic halo (Bond 1980; Beers et al. 1985, 1992; Norris et al. 1999, and additional references from Fulbright 2000). These abundance measurements are based on equivalent width analysis and/or spectral synthesis. Not all of the hundreds of stars in these studies could be observed. The highest priority was assigned to the most metal-poor stars.

Strictly speaking, Lai et al. (2004, 2007) did not conduct an HRS study because their Keck/ESI spectra had a spectral resolution of $R \sim 7000$. We still include their sample in the comparison along with the truly high-resolution studies.

\subsection{Dwarf Spheroidal Galaxies}

\subsubsection{Sculptor}

Paper I describes in detail the target selection for stars in the Sculptor dSph. For convenience, we reproduce here the map of targets on the sky and the Sculptor CMD (Figure 1).

\subsubsection{Fornax}

We adopted the $B R$ photometric catalog of Stetson et al. (1998), and we selected targets based on position in the CMD. Magnitudes were corrected star by star for extinction determined from the Schlegel et al. (1998) dust maps. (The same dust maps were not used for GCs because GCs subtend a smaller solid angle than dSphs. Therefore, we used a single value for each GC rather than interpolation in the Schlegel et al. dust maps.) Targets were drawn from seven polygons surrounding the RGB and horizontal branch. In order from highest to lowest priority, the targets were selected (1) from $R_{0} \leqslant 18.3$ to the tip of the RGB, (2) $18.3 \leqslant R_{0}<19.0$, (3) $19.0 \leqslant R_{0}<19.5$, (4) $19.5 \leqslant R_{0}<20.0$, and (5) $20.0 \leqslant R_{0}<20.5$. The red and blue edges of the polygons extended until the stellar density reached the background level, with a typical width of $\Delta(B-R)_{0}=0.9$ centered on the RGB.

Figure 2 shows the Fornax field with the spectroscopic targets highlighted in red. To minimize confusion, only targets with $R_{0}<20.5$ are plotted. The five DEIMOS slitmasks are for1B, for3B, for4B, for6, and for7 (see Table 2). Each slitmask except for4B included at least one of 18 duplicate targets included on other slitmasks. 
Table 2

DEIMOS Observations

\begin{tabular}{|c|c|c|c|c|c|c|}
\hline Object & Slitmask & No. of Targets & Date & Airmass & Seeing & Exposures \\
\hline \multicolumn{7}{|c|}{ Globular clusters } \\
\hline \multirow[t]{2}{*}{ NGC 288} & $\mathrm{n} 288$ & 119 & 2008 Nov 24 & 1.92 & $1^{\prime \prime} .3$ & $300 \mathrm{~s}, 2 \times 420 \mathrm{~s}$ \\
\hline & & & 2008 Nov 25 & 1.86 & $1^{\prime \prime} 23$ & $4 \times 300 \mathrm{~s}$ \\
\hline \multirow[t]{2}{*}{ M79 } & $\mathrm{n} 1904^{\mathrm{a}}$ & 22 & 2006 Feb 2 & 1.42 & Unknown & $2 \times 300 s$ \\
\hline & ng1904 & 104 & 2009 Feb 22 & 1.40 & $1^{\prime \prime} 12$ & $2 \times 600 \mathrm{~s}, 2 \times 1200 \mathrm{~s}$ \\
\hline \multirow[t]{3}{*}{ NGC 2419} & $\mathrm{n} 2419^{\mathrm{a}}$ & 70 & 2006 Feb 2 & 1.21 & Unknown & $4 \times 300 \mathrm{~s}$ \\
\hline & $\mathrm{n} 2419 \mathrm{c}$ & 94 & 2009 Oct 13 & 1.15 & 0.56 & $1200 \mathrm{~s}, 900 \mathrm{~s}$ \\
\hline & & & 2009 Oct 14 & 1.15 & 0.51 & $200 \mathrm{~s}, 900 \mathrm{~s}$ \\
\hline M5 & ng5904 & 181 & 2009 Feb 22 & 1.05 & 0.64 & $1200 \mathrm{~s}, 900 \mathrm{~s}, 600 \mathrm{~s}, 480 \mathrm{~s}$ \\
\hline M13 & n6205 & 93 & 2007 Oct 12 & 1.35 & Unknown & $3 \times 300 \mathrm{~s}$ \\
\hline M92 & LVMslits & 1 & 2008 May 6 & 1.09 & Unknown & $500 \mathrm{~s}, 2 \times 300 \mathrm{~s}$ \\
\hline M71 & n6838 & 104 & 2007 Nov 13 & 1.09 & 0.6 & $3 \times 300 \mathrm{~s}$ \\
\hline NGC 7006 & n7006 & 105 & 2007 Nov 15 & 1.01 & 0.57 & $2 \times 300 s$ \\
\hline \multirow[t]{3}{*}{ M15 } & n7078 & 63 & 2007 Nov 14 & 1.01 & 0.77 & $2 \times 300 s$ \\
\hline & n7078d & 164 & 2009 Oct 13 & 1.01 & 0.53 & $3 \times 900 \mathrm{~s}$ \\
\hline & $\mathrm{n} 7078 \mathrm{e}$ & 167 & 2009 Oct 14 & 1.01 & 0.61 & $3 \times 900 \mathrm{~s}$ \\
\hline M2 & n7089b & 91 & 2009 Oct 13 & 1.09 & $0 ! 57$ & $3 \times 900 \mathrm{~s}$ \\
\hline \multirow[t]{2}{*}{ Pal 13} & pal13 & 33 & 2009 Oct 13 & 1.48 & 0.60 & $2 \times 900 \mathrm{~s}$ \\
\hline & & & 2009 Oct 14 & 1.50 & 0.74 & $900 \mathrm{~s}, 822 \mathrm{~s}$ \\
\hline NGC 7492 & n7492 & 38 & 2007 Nov 15 & 1.30 & 0.57 & $2 \times 210 \mathrm{~s}$ \\
\hline \multicolumn{7}{|c|}{ Halo field stars } \\
\hline HE 0012-1441 & LVMslits & 1 & 2010 Aug 11 & 1.75 & $>1^{\prime \prime} .5$ & $2 \times 900 \mathrm{~s}$ \\
\hline HD 88609 & LVMslits & 1 & 2008 May 6 & 1.22 & Unknown & $30 \mathrm{~s}, 2 \times 100 \mathrm{~s}$ \\
\hline HD 115444 & LVMslits & 1 & 2008 May 6 & 1.04 & Unknown & $200 \mathrm{~s}, 100 \mathrm{~s}$ \\
\hline BS 16467-062 & LVMslits & 1 & 2008 May 6 & 1.00 & Unknown & $4 \times 1000 \mathrm{~s}$ \\
\hline HD 122563 & LVMslits & 1 & 2008 May 6 & 1.20 & Unknown & $3 \times 5 s$ \\
\hline BS 16550-087 & LVMslits & 1 & 2008 May 6 & 1.04 & Unknown & $4 \times 1000 \mathrm{~s}$ \\
\hline CS $30325-028$ & LVMslits & 1 & 2008 May 6 & 1.10 & Unknown & $3 \times 500 \mathrm{~s}$ \\
\hline CS 30329-129 & LongMirr & 1 & 2008 Apr 11 & 1.52 & Unknown & $2 \times 120 \mathrm{~s}$ \\
\hline $\mathrm{BD}+53098$ & LVMslits & 1 & 2008 May 6 & 1.07 & Unknown & $3 \times 50 \mathrm{~s}$ \\
\hline BS $16084-160$ & LongMirr & 1 & 2008 Apr 11 & 1.29 & Unknown & $2 \times 60 s$ \\
\hline $\mathrm{BD}+93223$ & LVMslits & 1 & 2008 May 6 & 1.02 & Unknown & $3 \times 50 \mathrm{~s}$ \\
\hline BS 16080-054 & LongMirr & 1 & 2008 Apr 11 & 1.36 & Unknown & $3 \times 120 \mathrm{~s}$ \\
\hline CS 22878-101 & LongMirr & 1 & 2008 Apr 8 & 1.10 & Unknown & $3 \times 120 \mathrm{~s}$ \\
\hline BS $16080-093$ & LongMirr & 1 & 2008 Apr 11 & 1.33 & Unknown & $3 \times 120 s$ \\
\hline $\mathrm{BD}+233130$ & LongMirr & 1 & 2008 Apr 11 & 1.00 & Unknown & $2 \times 45 \mathrm{~s}$ \\
\hline HD 165195 & LVMslits & 1 & 2008 May 6 & 1.05 & Unknown & $3 \times 10 \mathrm{~s}$ \\
\hline HD 186478 & LVMslits & 1 & 2008 May 6 & 1.47 & Unknown & $2 \times 50 \mathrm{~s}, 300 \mathrm{~s}$ \\
\hline $\mathrm{BD}-185550$ & LVMslits & 1 & 2008 May 6 & 1.30 & Unknown & $3 \times 100 \mathrm{~s}$ \\
\hline $\mathrm{BD}-176036$ & LVMslits & 1 & 2008 May 6 & 1.47 & Unknown & $3 \times 200 \mathrm{~s}$ \\
\hline CS 22880-086 & LVMslits & 1 & 2008 May 6 & 1.40 & Unknown & $4 \times 1000 \mathrm{~s}$ \\
\hline HE 2323-0256 & LVMslits & 1 & 2010 Aug 11 & 1.18 & $>1^{\prime \prime} .5$ & $2 \times 600 \mathrm{~s}$ \\
\hline \multicolumn{7}{|c|}{ dSphs } \\
\hline \multirow[t]{6}{*}{ Sculptor } & scl1 & 86 & 2008 Aug 3 & 1.79 & 0.85 & $3 \times 1200 \mathrm{~s}$ \\
\hline & $\mathrm{scl} 2$ & 106 & 2008 Aug 3 & 1.68 & 0.85 & $2 \times 900 \mathrm{~s}$ \\
\hline & scl3 & 87 & 2008 Aug 4 & 1.67 & $0^{\prime} 94$ & $462 \mathrm{~s}$ \\
\hline & & & 2008 Aug 31 & 1.67 & 0.77 & $1000 \mathrm{~s}, 834 \mathrm{~s}$ \\
\hline & scl5 & 95 & 2008 Sep 1 & 1.73 & 0.84 & $3 \times 720 s$ \\
\hline & sc16 & 91 & 2008 Sep 1 & 1.88 & $1^{\prime \prime} 23$ & $3 \times 720 s$ \\
\hline \multirow[t]{8}{*}{ Fornax } & for1B & 166 & 2008 Sep 1 & 2.00 & $1^{\prime \prime} 08$ & $3 \times 500 \mathrm{~s}$ \\
\hline & & & 2008 Nov 25 & 1.86 & $0 ! 7$ & $2 \times 1200 \mathrm{~s}$ \\
\hline & for3B & 169 & 2008 Sep 1 & 1.71 & 0.85 & $3 \times 500 \mathrm{~s}$ \\
\hline & for $4 B$ & 164 & 2008 Nov 26 & 1.74 & $1^{\prime \prime} .1$ & $1200 \mathrm{~s}, 1020 \mathrm{~s}$ \\
\hline & for6 & 169 & 2008 Aug 31 & 1.73 & 0.68 & $3 \times 500 \mathrm{~s}$ \\
\hline & & & 2008 Nov 25 & 2.31 & $1^{\prime \prime} .2$ & $2 \times 1200 \mathrm{~s}$ \\
\hline & for7 & 169 & 2008 Aug 31 & 1.71 & 0.76 & $2 \times 500 \mathrm{~s}, 460 \mathrm{~s}$ \\
\hline & & & 2008 Sep 30 & 1.71 & Unknown & $3 \times 600 \mathrm{~s}$ \\
\hline \multirow[t]{7}{*}{ Leo I } & LeoI_1 ${ }^{b}$ & 42 & 2003 Oct 29 & 1.86 & Unknown & $4800 \mathrm{~s}$ total \\
\hline & LeoI_2 ${ }^{b}$ & 83 & 2004 Oct 15 & & Unknown & $6900 \mathrm{~s}$ total \\
\hline & LIN1_1 & 112 & $2006 \mathrm{Feb} 2$ & 1.82 & Unknown & $3600 \mathrm{~s}$ total \\
\hline & LIN1_2 & 100 & 2006 Feb 2 & 1.23 & Unknown & $3600 \mathrm{~s}$ total \\
\hline & LIN1_3 & 92 & $2006 \mathrm{Feb} 2$ & 1.04 & Unknown & $3600 \mathrm{~s}$ total \\
\hline & LIN1_4 & 100 & 2006 Feb 2 & 1.01 & Unknown & $2520 \mathrm{~s}$ total \\
\hline & LIN2_1 & 104 & 2006 Feb 3 & 1.83 & Unknown & $4800 \mathrm{~s}$ total \\
\hline
\end{tabular}


Table 2

(Continued)

\begin{tabular}{|c|c|c|c|c|c|c|}
\hline Object & Slitmask & No. of Targets & Date & Airmass & Seeing & Exposures \\
\hline & LIN2_2 & 98 & 2006 Feb 3 & 1.20 & Unknown & $4800 \mathrm{~s}$ total \\
\hline & LIN2_3 & 67 & 2006 Feb 3 & 1.01 & Unknown & $3600 \mathrm{~s}$ total \\
\hline & LIN2_4 & 102 & 2006 Feb 3 & 1.07 & Unknown & $3600 \mathrm{~s}$ total \\
\hline & LIN3_1 & 86 & 2006 Feb 4 & 1.43 & Unknown & $3011 \mathrm{~s}$ total \\
\hline & LIN3_2 & 67 & 2006 Feb 4 & 1.21 & Unknown & $4800 \mathrm{~s}$ total \\
\hline & LIN3_3 & 69 & 2006 Feb 4 & 1.02 & Unknown & $4500 \mathrm{~s}$ total \\
\hline & LIN3_4 & 88 & 2006 Feb 4 & 1.04 & Unknown & $1800 \mathrm{~s}$ total \\
\hline \multirow[t]{5}{*}{ Sextans } & $\operatorname{sex} 1$ & 108 & 2009 Feb 22 & 1.53 & $0 ! 96$ & $3 \times 1200 \mathrm{~s}, 396 \mathrm{~s}$ \\
\hline & $\operatorname{sex} 2$ & 85 & 2009 Feb 22 & 1.21 & 0.70 & $3 \times 1200 \mathrm{~s}$ \\
\hline & $\operatorname{sex} 3$ & 88 & 2009 Feb 22 & 1.09 & 0.93 & $4 \times 1200 \mathrm{~s}$ \\
\hline & $\operatorname{sex} 4$ & 109 & 2009 Feb 23 & 1.51 & 1".06 & $4 \times 1200 \mathrm{~s}$ \\
\hline & $\operatorname{sex} 6$ & 100 & 2009 Feb 23 & 1.19 & 0.86 & $3 \times 1200 \mathrm{~s}$ \\
\hline \multirow[t]{6}{*}{ Leo II } & L2A & 72 & $2006 \mathrm{Feb} 2$ & 1.00 & Unknown & $3300 \mathrm{~s}$ total \\
\hline & L2B & 56 & 2006 Feb 3 & 1.29 & Unknown & $1080 \mathrm{~s}$ total \\
\hline & $\mathrm{L} 2 \mathrm{C}$ & 77 & 2006 Feb 4 & 1.01 & Unknown & $3960 \mathrm{~s}$ total \\
\hline & L2D & 70 & 2006 Feb 2 & 1.09 & Unknown & $3600 \mathrm{~s}$ total \\
\hline & L2E & 57 & 2006 Feb 3 & 1.07 & Unknown & $3600 \mathrm{~s}$ total \\
\hline & $\mathrm{L} 2 \mathrm{~F}$ & 62 & 2006 Feb 4 & 1.13 & Unknown & $3400 \mathrm{~s}$ total \\
\hline \multirow[t]{4}{*}{ Canes Venatici I } & CVn1-1 ${ }^{\mathrm{a}}$ & 91 & 2007 Feb 14 & 1.14 & Unknown & $4140 \mathrm{~s}$ total \\
\hline & $\mathrm{CVn} 1-2^{\mathrm{a}}$ & 94 & 2007 Feb 14 & 1.06 & Unknown & $4140 \mathrm{~s}$ total \\
\hline & CVn1-3 $3^{\mathrm{a}}$ & 90 & 2007 Feb 14 & 1.03 & Unknown & $4860 \mathrm{~s}$ total \\
\hline & CVn1-dp ${ }^{a}$ & 115 & 2007 Feb 15 & 1.03 & Unknown & $9000 \mathrm{~s}$ total \\
\hline \multirow[t]{4}{*}{ Ursa Minor } & umi1 & 125 & 2009 Feb 22 & 1.99 & 0.71 & $3 \times 1200 \mathrm{~s}$ \\
\hline & umi2 & 134 & 2009 Feb 22 & 1.79 & $1^{\prime \prime} 00$ & $2 \times 1200 \mathrm{~s}, 1400 \mathrm{~s}$ \\
\hline & umi3 & 137 & 2009 Feb 23 & 1.48 & 0.98 & $3 \times 1200 \mathrm{~s}$ \\
\hline & umi6 & 137 & 2009 Feb 23 & 1.57 & $0 ! 93$ & $3 \times 1200 \mathrm{~s}$ \\
\hline \multirow[t]{8}{*}{ Draco } & dra1 & 151 & 2009 May 23 & 1.27 & 0.67 & $3 \times 1200 \mathrm{~s}$ \\
\hline & dra2 & 167 & 2009 May 23 & 1.28 & 0.74 & $1200 \mathrm{~s}, 1000 \mathrm{~s}, 900 \mathrm{~s}$ \\
\hline & dra3 & 140 & 2009 May 23 & 1.35 & 0.73 & $3 \times 960 \mathrm{~s}$ \\
\hline & dra4 & 140 & 2009 May 23 & 1.50 & 0.63 & $2 \times 960 \mathrm{~s}, 700 \mathrm{~s}$ \\
\hline & dra5 & 78 & 2009 May 24 & 1.28 & 0.69 & $3 \times 1200 \mathrm{~s}$ \\
\hline & dra7 & 75 & 2009 May 24 & 1.35 & $0 ! 75$ & $3 \times 960 \mathrm{~s}, 1080 \mathrm{~s}$ \\
\hline & dra8 & 60 & 2009 May 24 & 1.55 & 0.57 & $3 \times 840 \mathrm{~s}$ \\
\hline & dra9 & 106 & 2009 May 24 & 1.28 & 0.66 & $3 \times 960 \mathrm{~s}$ \\
\hline
\end{tabular}

Notes.

a Observations by Simon \& Geha (2007).

${ }^{\mathrm{b}}$ Observations by Sohn et al. (2007).

Figure 2 also shows the CMD of the targets within the right ascension and declination ranges of the axes in the left panel of Figure 2. Some stars on the extreme blue end of the RGB did not pass the spectroscopic selection criteria. These stars may be asymptotic giant branch stars, or they may be extremely young or extremely metal-poor red giants. This potential selection bias should be kept in mind when considering the derived metallicity distribution of Fornax.

Twenty stars, listed in Table 7, have previously published HRS abundance measurements (Shetrone et al. 2003; Letarte et al. 2010), and all 20 were observed. One of these stars, M12 (not shown in the right panel of Figure 2), was absent from the $B R$ photometric catalog. For calculation of photometric temperature and surface gravity, we adopted the same extinction-corrected VI magnitudes used by Shetrone et al. (2003).

\subsubsection{Leo I}

We used Sohn et al.'s (2007) DEIMOS spectra of individual stars in Leo I and $M T_{2}$ magnitudes of the spectroscopic targets. We converted $M$ and $T_{2}$ to Cousins $V$ and $I$ magnitudes in the same manner as for the Sculptor photometric catalog. Magnitudes were corrected star by star for extinction determined from the Schlegel et al. (1998) dust maps. For other details on the photometric catalog and spectroscopic target selection, we refer to Sohn et al.'s published paper on the DEIMOS slitmasks LeoI_1 and LeoI_2.

Figure 3 shows the sky chart and CMD for spectroscopic targets. The black points in the right panel of the figure show other stars from the VI catalog of Bellazzini et al. (2004) for context, but the spectroscopic targets were not drawn from this catalog. Shetrone et al. (2003) measured spectroscopic abundances of two stars in Leo I. One of these, M5, was observed on three different DEIMOS slitmasks.

\subsubsection{Sextans}

We selected spectroscopic targets from the deep, wide-field $B V I$ catalog of Lee et al. (2003). They assumed a constant reddening of $E(B-V)=0.01$, and we corrected all of the magnitudes and colors accordingly. We selected RGB members by overlaying Yonsei-Yale isochrones (Demarque et al. 2004) between 2 and $14 \mathrm{Gyr},[\mathrm{Fe} / \mathrm{H}]=-3.76$ and +0.05 , and $[\alpha / \mathrm{Fe}]=0.0$ and +0.3 on the CMD. All stars that lay between the bluest and reddest of these isochrones within photometric errors were considered for spectroscopic selection. An additional 0.05 mag was allowed on the blue edge of the bluest isochrones to account for possible systematic error in the isochrones which might have excluded extremely metal-poor 
$\Delta x(\mathrm{pc})$
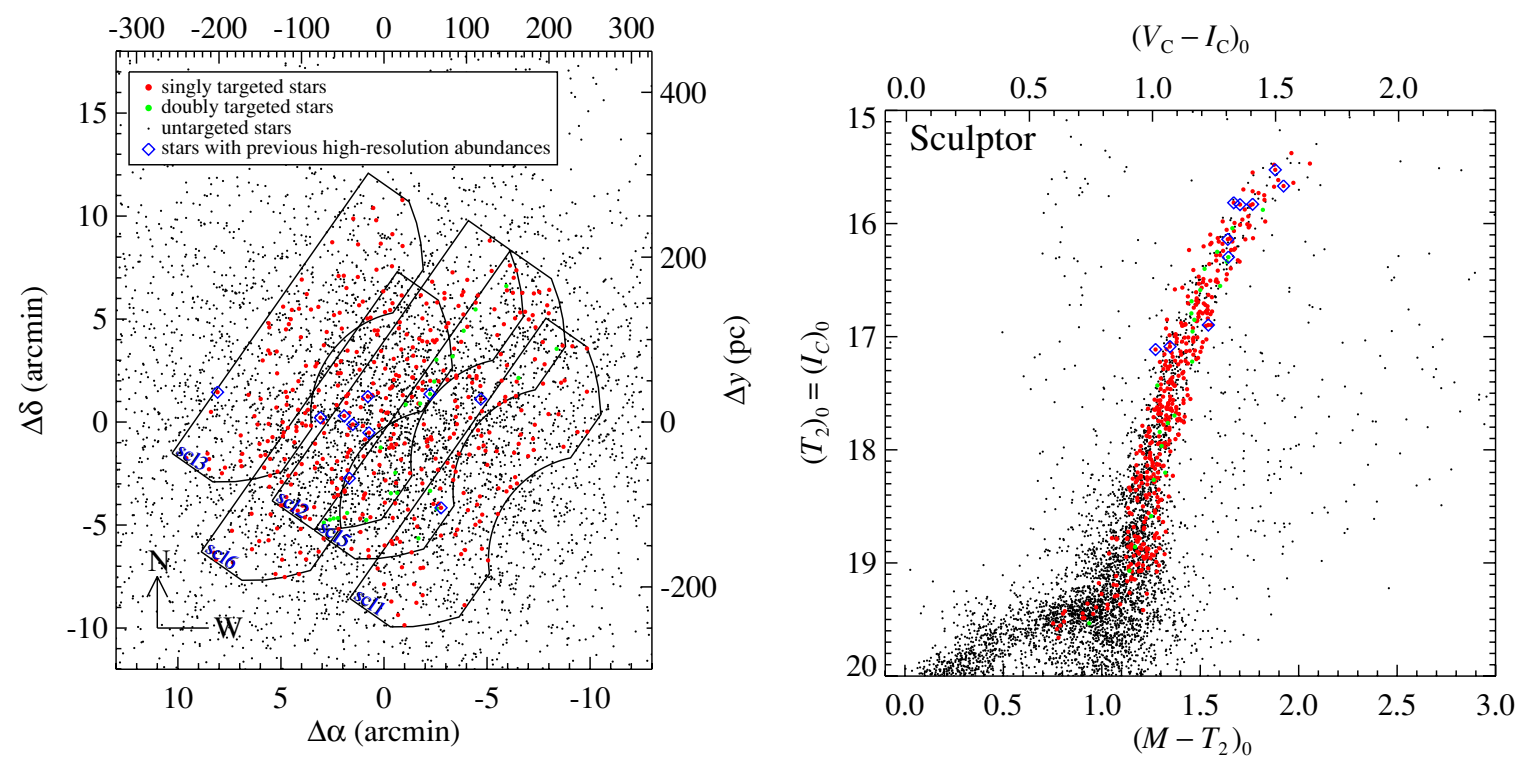

Figure 1. Sculptor. Left: DEIMOS slitmask footprints laid over a map of sources from the Sculptor photometric catalog (Westfall et al. 2006). Targets selected for spectroscopy are shown in red. Targets observed in more than one mask are shown in green. Blue diamonds enclose stars with previous HRS abundance measurements. Each slitmask is labeled in blue with the names given in Table 2. The left and bottom axis scales show the angular displacement in arcmin from the center of the galaxy $\left(\alpha_{0}=1^{\mathrm{h}} 00^{\mathrm{m}} 09^{\mathrm{s}}, \delta_{0}=-33^{\circ} 42^{\prime} \cdot 5\right.$; Mateo 1998), and the right and top axis scales show the projected physical distance for an assumed distance to Sculptor of $85.9 \mathrm{kpc}$ (Pietrzyński et al. 2008). Right: extinction- and reddening-corrected color-magnitude diagram in the Washington and Cousins systems for the photometric sources within the right ascension and declination ranges shown at left. The transformation from the Washington system $\left(M\right.$ and $\left.T_{2}\right)$ to the Cousins system ( $V_{\mathrm{C}}$ and $I_{\mathrm{C}}$ ) is $I_{\mathrm{C}}=T_{2}$ and $V_{\mathrm{C}}-I_{\mathrm{C}}=0.800\left(M-T_{2}\right)-0.006$ (Majewski et al. 2000).

(A color version of this figure is available in the online journal.)
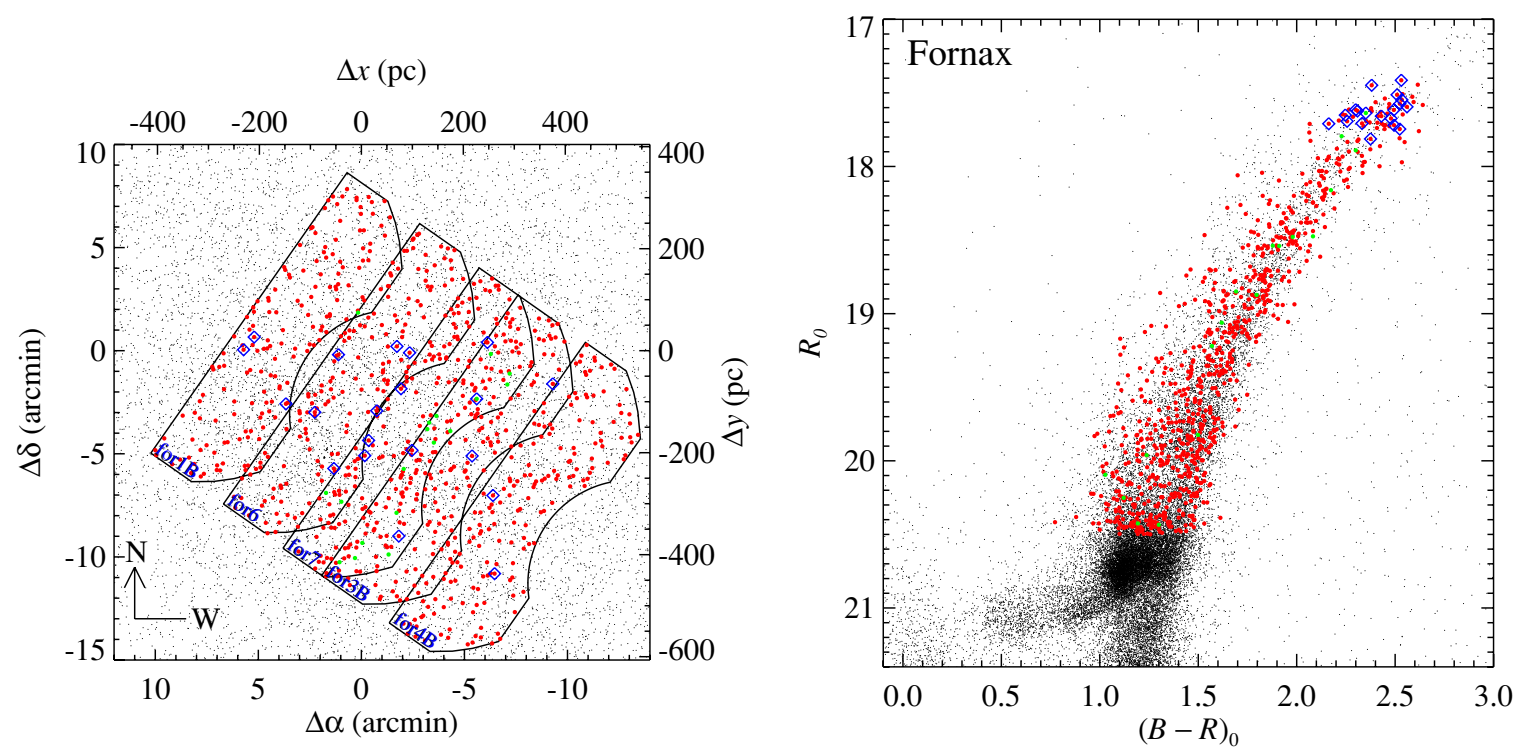

Figure 2. Fornax. Left: DEIMOS slitmask footprints laid over a map of sources from the Fornax photometric catalog (Stetson et al. 1998) with magnitude $R_{0}<20.5$ The center of the galaxy is $\alpha_{0}=2^{\mathrm{h}} 39^{\mathrm{m}} 59^{\mathrm{s}}, \delta_{0}=-34^{\circ} 27^{\prime} .0$ (Mateo 1998), and the distance is $139 \mathrm{kpc}$ (Rizzi et al. 2007). Right: color-magnitude diagram for the photometric sources within the right ascension and declination ranges shown at left. See Figure 1 for further explanation.

(A color version of this figure is available in the online journal.)

stars. When forced to choose between multiple RGB candidates, we selected the brightest one.

Figure 4 shows the Sextans field with the spectroscopic targets highlighted in red. Only targets with $I<22$ are plotted to minimize confusion. The sex 3 slitmask contained 25 targets also included on other slitmasks.

Figure 4 also shows the CMD of the targets within the right ascension and declination ranges of the axes in the left panel of Figure 4. Some extremely red stars were targeted in order to fill the slitmask with targets. Although these stars are unlikely to be Sextans members, they were included because they could potentially be metal-rich RGB stars in Sextans. Additionally, Sextans is near enough to permit a significant number of horizontal branch stellar spectra, although the spectroscopic abundance measurement technique used here does not yet work for horizontal branch stars. Five stars, listed in Table 7, have previously published HRS abundance measurements (Shetrone et al. 2001), and all five were observed. Aoki et al. (2009) 

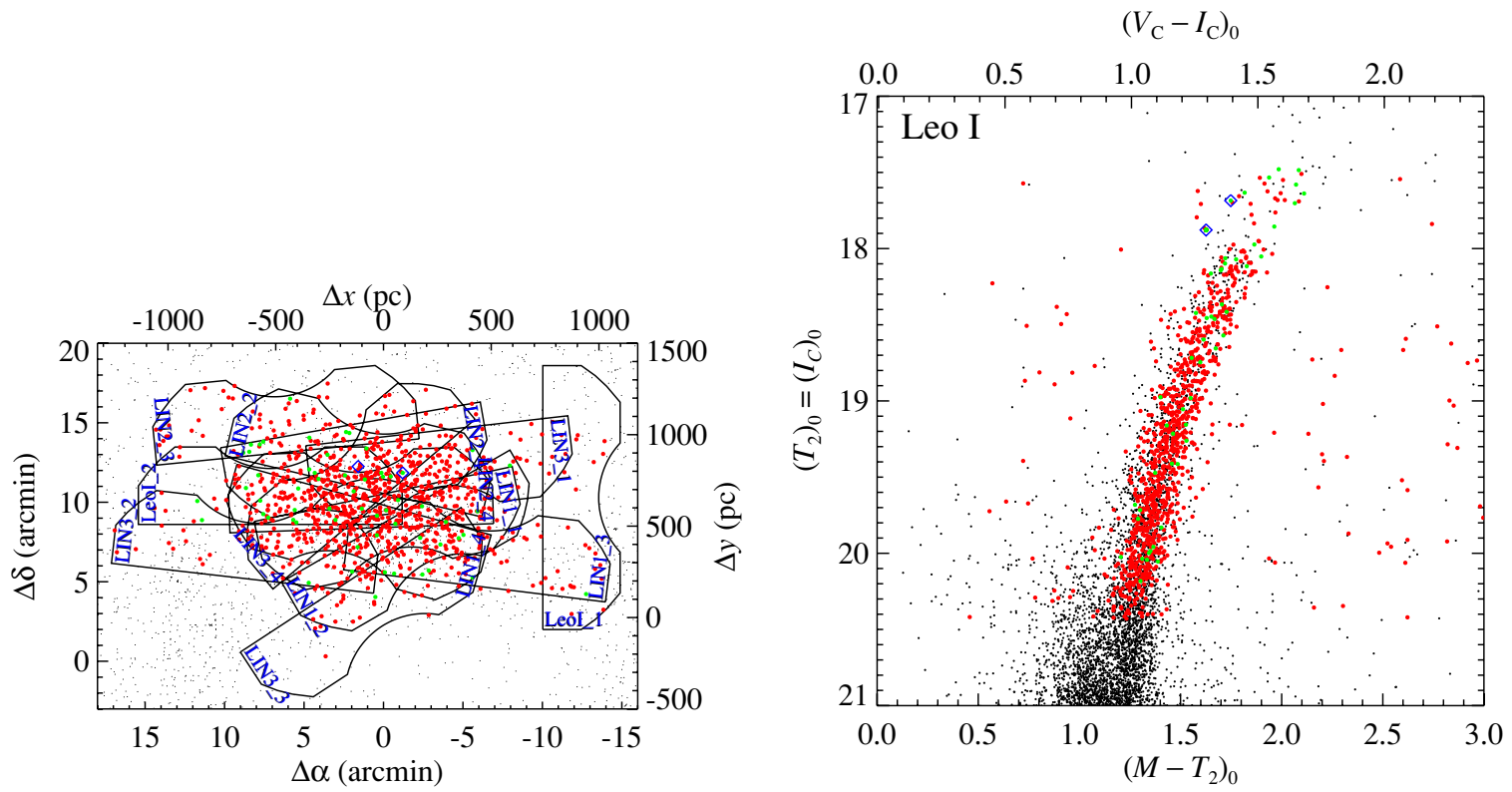

Figure 3. Leo I. Left: DEIMOS slitmask footprints laid over a map of sources from the NOMAD catalog (Zacharias et al. 2004), which is used only to show context of the sky area surrounding the DEIMOS targets. The center of the galaxy is $\alpha_{0}=10^{\mathrm{h}} 08^{\mathrm{m}} 27^{\mathrm{s}}, \delta_{0}=12^{\circ} 08.5$ (Mateo 1998), and the distance is $254 \mathrm{kpc}$ (Bellazzini et al. 2004). Right: colors and magnitudes of the spectroscopic targets (red points). Colors and magnitudes of the spectroscopically untargeted stars (black points) come from the catalog of Bellazzini et al. (2004), which is used only to show context in the color-magnitude diagram. Only one of the two HRS targets was observed with DEIMOS. See Figure 1 for further explanation.

(A color version of this figure is available in the online journal.)
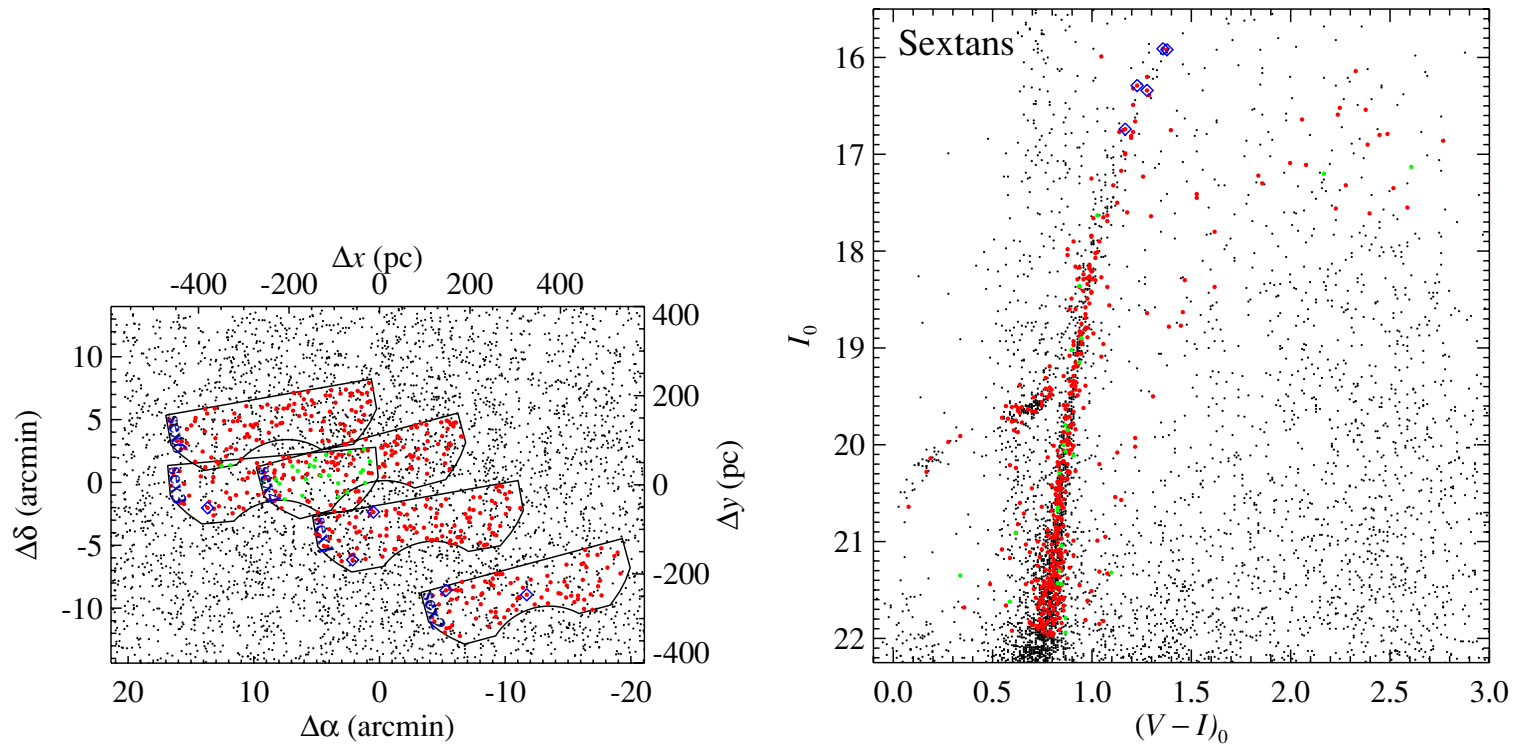

Figure 4. Sextans. Left: DEIMOS slitmask footprints laid over a map of sources from the Sextans photometric catalog (Lee et al. 2003) with magnitude $I<22$. The center of the galaxy is $\alpha_{0}=10^{\mathrm{h}} 13^{\mathrm{m}} 03^{\mathrm{s}}, \delta_{0}=-01^{\circ} 36^{\prime} .9$ (Mateo 1998), and the distance is $95.5 \mathrm{kpc}$ (Lee et al. 2003). Right: color-magnitude diagram for the photometric sources within the right ascension and declination ranges shown at left. See Figure 1 for further explanation.

(A color version of this figure is available in the online journal.)

published measurements of an additional six stars at high resolution after we designed the DEIMOS slitmasks. These stars were not included in our slitmasks.

\subsubsection{Leo II}

DEIMOS observations of Leo II were conducted in the same program as observations of Leo I. The photometry and spectroscopy were treated identically. For additional information, see Section 2.3.3. Figure 5 shows the spectroscopic target selection.

\subsubsection{Canes Venatici I}

We used Simon \& Geha's (2007) DEIMOS spectra for individual stars in Canes Venatici I. Photometry and extinction corrections were taken from the SDSS Data Release 5 (Adelman-McCarthy et al. 2007). In order to use the same isochrones for determining effective temperatures and surface 


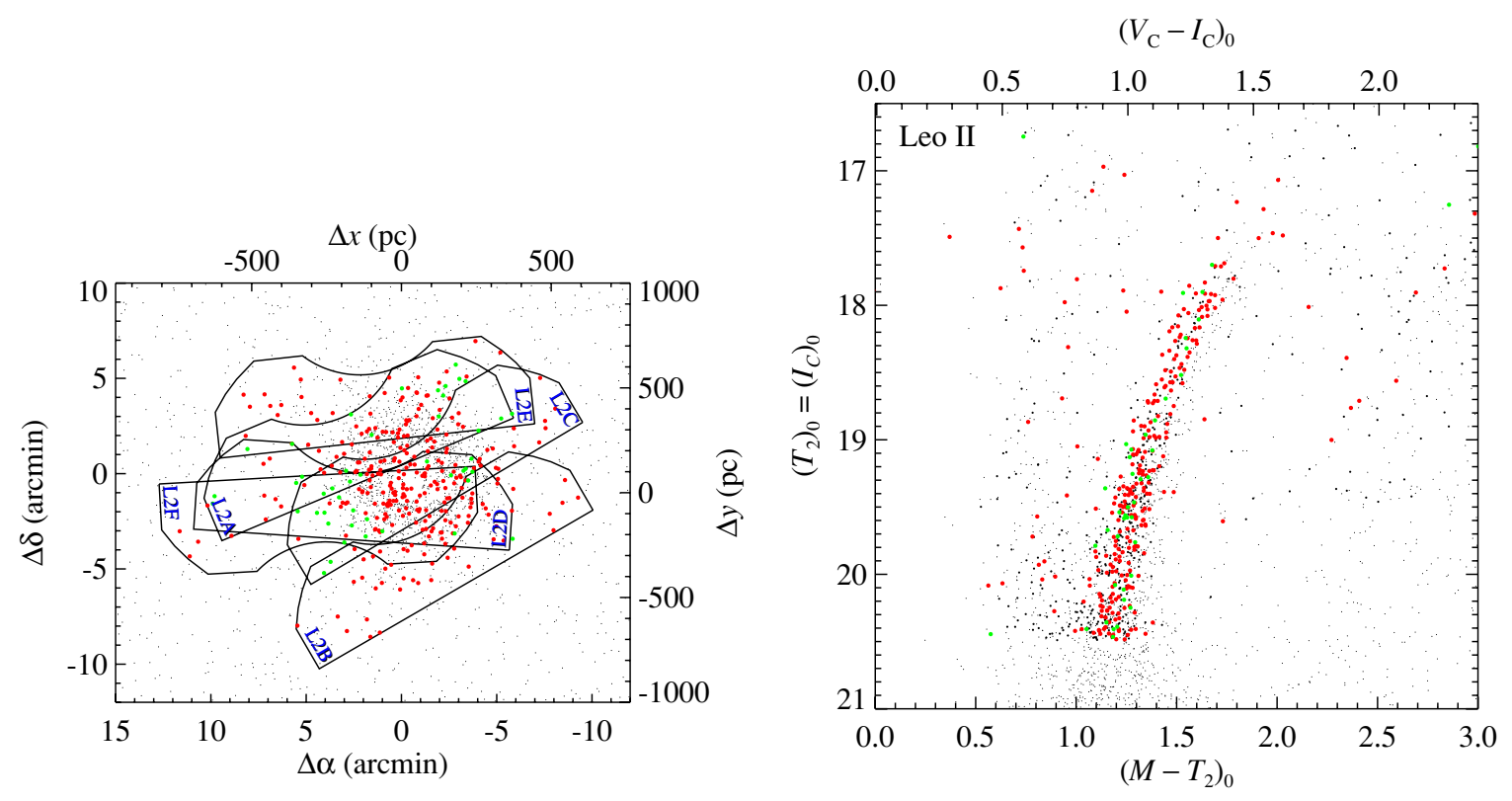

Figure 5. Leo II. Left: DEIMOS slitmask footprints laid over a map of stellar sources from the SDSS DR5 photometric catalog (Adelman-McCarthy et al. 2007), which is used only to show context of the sky area surrounding the DEIMOS targets. The center of the galaxy is $\alpha_{0}=11^{\mathrm{h}} 13^{\mathrm{m}} 29^{\mathrm{s}}, \delta_{0}=+22^{\circ} 09$.'2 $($ Mateo 1998$)$, and the distance is $219 \mathrm{kpc}$ (Siegel et al. 2010). Right: colors and magnitudes of the spectroscopic targets (red points) from the photometric catalog of Siegel et al. (2010). Colors and magnitudes of the spectroscopically untargeted stars (black points) come from the SDSS DR5 catalog, which is used only to show context in the color-magnitude diagram. The SDSS ugriz magnitudes have been roughly converted to the Washington $M T_{2}$ system, causing a shift between the red and black points. See Figure 1 for further explanation.

(A color version of this figure is available in the online journal.)
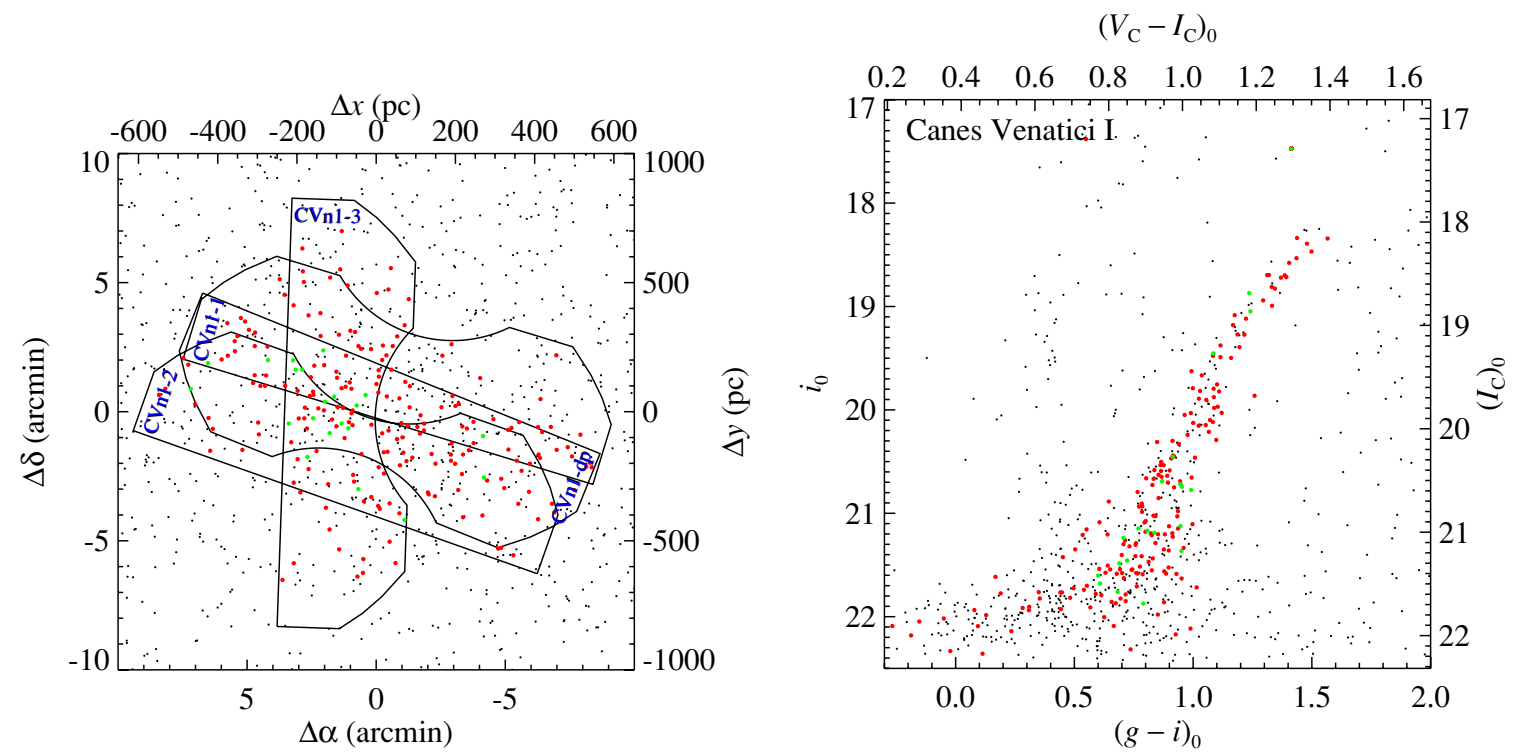

Figure 6. Canes Venatici I. Left: DEIMOS slitmask footprints laid over a map of stellar sources from the SDSS DR5 photometric catalog (Adelman-McCarthy et al. 2007). The center of the galaxy is $\alpha_{0}=13^{\mathrm{h}} 28^{\mathrm{m}} 04^{\mathrm{s}}, \delta_{0}=+33^{\circ} 33^{\prime} 21^{\prime \prime}$ (Zucker et al. 2006), and the distance is $210 \mathrm{kpc}$ (Kuehn et al. 2008). Right: color-magnitude diagram for the photometric sources within the right ascension and declination ranges shown at left. The top and left axes give rough Cousins VI magnitudes, assuming average colors on the RGB and following the transformations of Jordi et al. (2006). See Figure 1 for further explanation.

(A color version of this figure is available in the online journal.)

gravities as we used for other galaxies, we converted SDSS ugriz to Johnson-Cousins UBVRI following the global, metallicityindependent transformations of Jordi et al. (2006). ${ }^{12}$ We refer to Simon \& Geha's article for the details of the spectroscopic

12 Kirby et al. (2008b) chose the conversions given by Chonis \& Gaskell (2008). However, Chonis \& Gaskell suggest that their purpose was to derive photometric zero points, not to find relations valid for astrophysical sources. Therefore, we relied on Jordi et al.'s (2006) transformations, which are valid for astrophysical sources. target selection, shown in Figure 6. No stars in Canes Venatici I have been observed yet at high spectral resolution.

\subsubsection{Ursa Minor}

We selected spectroscopic targets from the VI catalog of Bellazzini et al. (2002). Magnitudes were corrected star by star for extinction determined from the Schlegel et al. (1998) dust maps. We selected RGB members by following the same procedure as for Sextans, except that we allowed an additional 

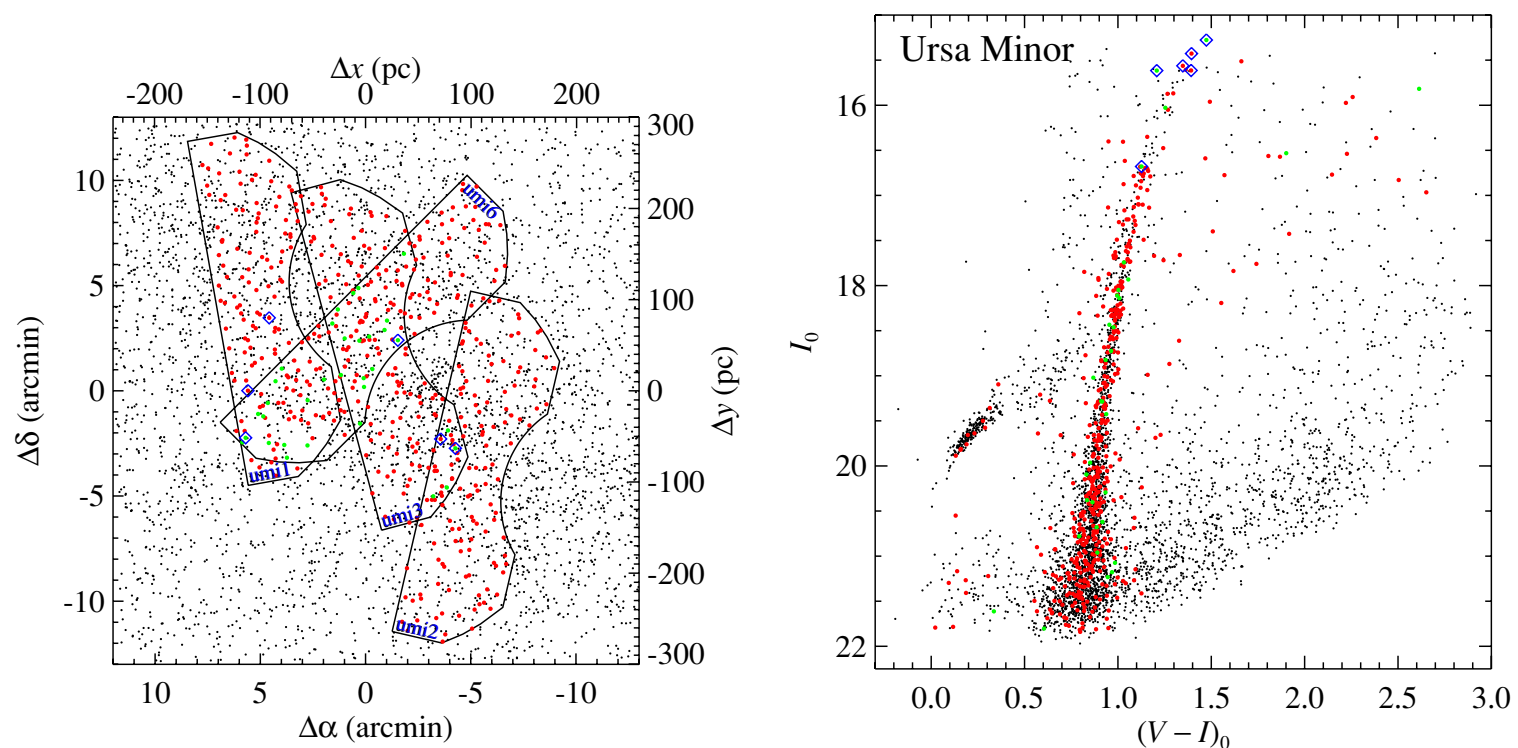

Figure 7. Ursa Minor. Left: DEIMOS slitmask footprints laid over a map of sources from the Ursa Minor photometric catalog (Bellazzini et al. 2002). The center of the galaxy is $\alpha_{0}=15^{\mathrm{h}} 09^{\mathrm{m}} 11^{\mathrm{s}}, \delta_{0}=+67^{\circ} 12^{\prime} \cdot 9$ (Mateo 1998), and the distance is $69 \mathrm{kpc}$ (Mighell \& Burke 1999). Right: color-magnitude diagram for the photometric catalog (Bellazzini et al. 2002) sources within the right ascension and declination ranges shown at left. See Figure 1 for further explanation.

(A color version of this figure is available in the online journal.)

0.1 mag on the blue side of the RGB instead of 0.05 mag because the extra contamination was negligible.

Figure 7 shows the Ursa Minor field with the spectroscopic targets highlighted in red. Every slitmask contained several of the 33 targets also included on other slitmasks.

Figure 7 also shows the CMD of the targets within the right ascension and declination ranges of the axes in the left panel of Figure 7. The discussion of the Sextans CMD similarly applies to the Ursa Minor CMD. Six stars, listed in Table 7, have previously published HRS abundance measurements (Shetrone et al. 2001; Sadakane et al. 2004), and all six were observed in our study.

\subsubsection{Draco}

Ségall et al. (2007) devoted an extremely wide-field photometric survey to Draco. They observed Draco with three different telescopes, but we used only their gri catalog from the CFHT MegaCam Camera for its large field of view. In a manner similar to the target selection for Sextans, we chose targets from the $(g, g-i)$ CMD between the bluest and reddest Padova isochrones (Girardi et al. 2002) between 2 and $14 \mathrm{Gyr}$, $[\mathrm{Fe} / \mathrm{H}]=-2.23$ and +0.05 , and $[\alpha / \mathrm{Fe}]=0.0$ and +0.3 . We allowed an additional 0.05 mag on the blue side of the CMD to account for stars more metal-poor than the most metal-poor Padova isochrone $([\mathrm{Fe} / \mathrm{H}]=-2.23)$.

Figure 8 shows the large field on which eight Draco slitmasks were placed. Only stars with $i<22.5$ and $g-i<1.7$ are shown to minimize confusion. The dra7 and dra8 slitmasks were located on the periphery of the field to search for Draco members close to the tidal radius. The dra2 slitmask included 11 targets also observed on the dra1 and dra4 slitmasks.

Figure 8 also shows the CMD of targets that lay within the outlines of the slitmasks shown in the left panel of Figure 8. The discussion of the Sextans CMD similarly applies to the Draco CMD. Fourteen stars in Draco have been observed with HRS (Shetrone et al. 1998, 2001; Fulbright et al. 2004; Cohen $\&$ Huang 2009). We observed 12 of these. They are listed in Table 7.
We wished to use the same isochrones to determine effective temperature and surface gravities that we used for other galaxies. Clem et al. (2008) showed that CFHT gri magnitudes are indistinguishable from the USNO standard $g^{\prime} r^{\prime} i^{\prime}$ magnitudes. Therefore, we transformed CFHT gri magnitudes to SDSS gri magnitudes following the prescription of Tucker et al. (2006). Then, we transformed SDSS gri magnitudes to Johnson-Cousins BVRI magnitudes (Jordi et al. 2006). Regnault et al. (2009) give color transformations from $B V R I$ to CFHT griz but not absolute zero points for that conversion. Therefore, we used the twostep transformation. Because the CFHT and SDSS filter transmission curves are very similar, the first transformation from the CFHT system to the SDSS system introduced negligible error.

\subsubsection{Additional Galaxies}

The galaxies Ursa Major II, Leo IV, Coma Berenices, and Hercules are not included in the catalog presented here (Table 4). However, some stars in these four galaxies have been observed both with HRS and MRS. Like Canes Venatici I, these galaxies were observed with DEIMOS by Simon \& Geha (2007). Frebel et al. (2010b) observed at high resolution three stars each in Ursa Major II and Coma Berenices; Simon et al. (2010) observed at high resolution one star in Leo IV; and Koch et al. (2008) observed at high resolution two stars in Hercules, but only one overlaps our sample. We used these stars in the comparison between HRS and MRS abundance measurements (Section 4.4).

\subsection{Slitmask Design}

We designed the DEIMOS slitmasks with the dsimulator ${ }^{13}$ IRAF $^{14}$ software module. Each slitmask subtended approximately $16^{\prime} \times 5^{\prime}$. In order to subtract night sky emission lines

\footnotetext{
13 http://www.ucolick.org/ phillips/deimos_ref/masks.html

14 IRAF is distributed by the National Optical Astronomy Observatory, which is operated by the Association of Universities for Research in Astronomy, Inc., under cooperative agreement with the National Science Foundation.
} 

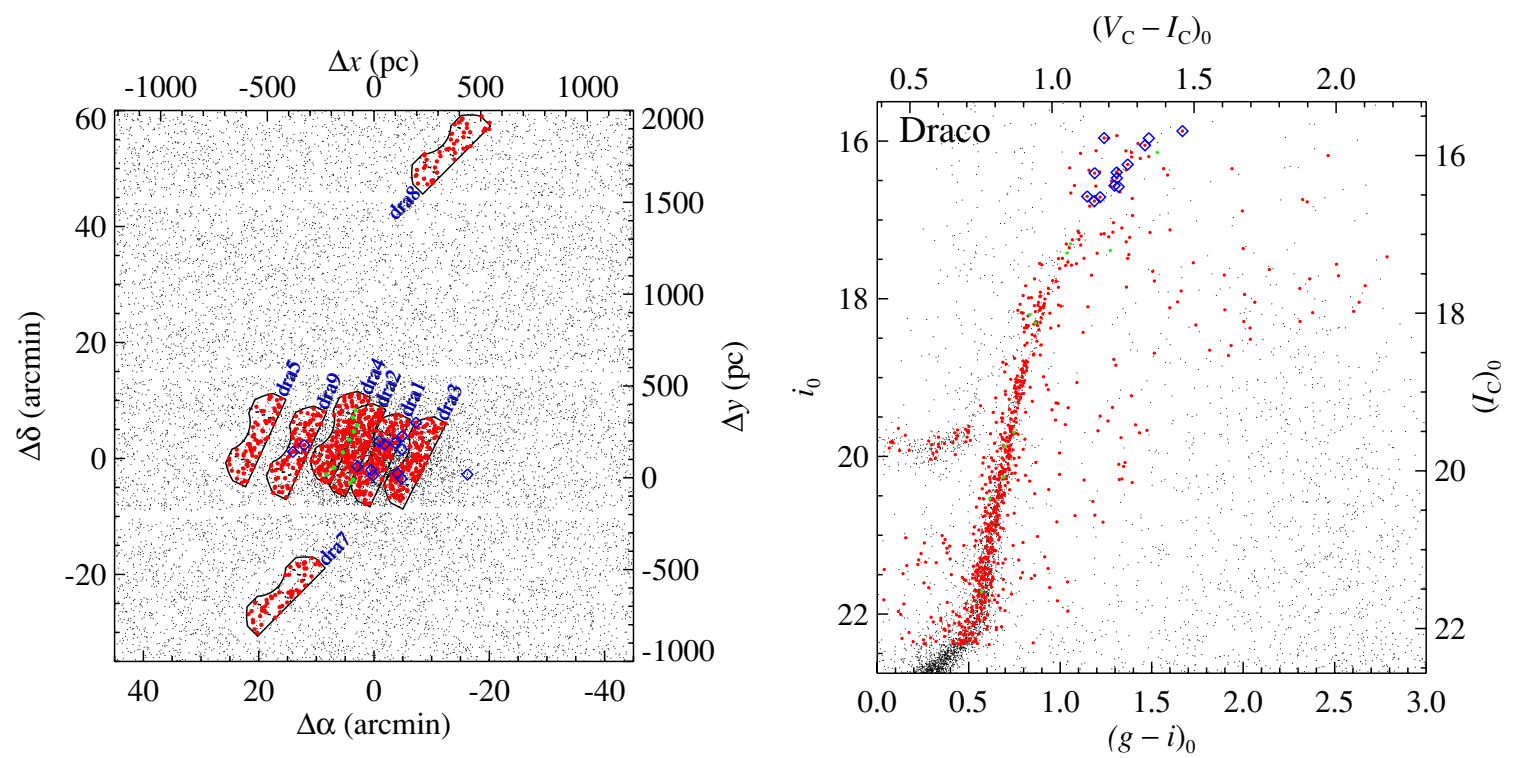

Figure 8. Draco. Left: DEIMOS slitmask footprints laid over a map of sources with $i<22.5$ and $g-i<1.7$ from the Draco photometric catalog (Ségall et al. 2007). The center of the galaxy is $\alpha_{0}=17^{\mathrm{h}} 20^{\mathrm{m}} 19^{\mathrm{s}}, \delta_{0}=+57^{\circ} 54^{\prime} .8$ (Mateo 1998), and the distance is $92.9 \mathrm{kpc}$ (Bellazzini et al. 2002). Right: color-magnitude diagram for the photometric sources within the outline of the slitmasks shown at left. The top and left axes give rough Cousins VI magnitudes, assuming average colors on the RGB and following the transformations of Tucker et al. (2006) and Jordi et al. (2006). See Figure 1 for further explanation.

(A color version of this figure is available in the online journal.)

adequately, we required a minimum slit length of $4^{\prime \prime}$. The minimum space between slits was 0'.35. Although this spacing is less than the typical seeing FWHM, light contamination between slitlets was negligible. Because slits had the freedom to be placed along the dispersion axis of the slitmask, the end of a slit was rarely located within $1^{\prime \prime}$ of a neighboring slit. Even in those rare instances, the stars were located far enough from the ends of their slits that light spillage into a neighboring slit did not affect the spectral extraction or sky subtraction.

When the slitmask design constraints forced the selection of one among multiple possible red giant candidates, we invoked priorities explained in Sections 2.1-2.3.8 (usually the brightest candidate). For most dSph slitmasks, the slitmasks were designed to be observed at approximately the parallactic angle at the anticipated time of observation. This choice minimized the small light losses due to differential atmospheric refraction. Most slitmasks' sky position angle (P.A.) was offset by $\sim 10^{\circ}$ from the slit P.A. The resulting tilt of the night sky emission lines relative to the CCD pixel grid increased the subpixel wavelength sampling and improved sky subtraction. The dSph slitmasks contained many duplicate observations, which provide estimates of uncertainties in abundance measurements (Section 4.3).

The spectral coverage of each slit is not the same. The minimum and maximum wavelengths of spectra of targets near the long, straight edge of the DEIMOS footprint can be up to $400 \AA$ lower than for targets near the irregularly shaped edge of the footprint (upper left and lower right of the slitmask footprints in Figure 1, respectively). Furthermore, spectra of targets near either extreme of the long axis of the slitmask suffered from vignetting, which reduced the spectral range. It is important to keep these differences of spectral range in mind when interpreting the differences of measurements derived from duplicate observations.

\subsection{Spectroscopic Configuration and Exposures}

Our observing strategy was nearly identical to that of Guhathakurta et al. (2006) and Simon \& Geha (2007). In summary, we used the 1200 lines $\mathrm{mm}^{-1}$ grating at a central wavelength of $\sim 7800 \AA$. The slit widths were 0 .' 7 (except for $1^{\prime \prime} .0$ slit widths for M79, NGC 2419, and Leo I and II), yielding a spectral resolution of $\sim 1.2 \AA$ FWHM (resolving power $R \sim 7000$ at $8500 \AA$ ). The OG550 filter blocked diffraction orders higher than $m=1$. The spectral range was about 6400-9000 $\AA$ with variation depending on the slit's location along the dispersion axis. Exposures of $\mathrm{Kr}, \mathrm{Ne}, \mathrm{Ar}$, and $\mathrm{Xe}$ arc lamps provided wavelength calibration, and exposures of a quartz lamp provided flat fielding. Table 2 lists the slitmasks observed, the number of targets on each slitmask, the dates of observations, the airmass, the approximate seeing (if known), and the exposure times. For the multi-slit masks, seeing was estimated by measuring the FWHM of a Gaussian fit to the one-dimensional profiles of alignment stars.

Because halo stars are not clustered on the sky, only one star could be observed in each exposure. Therefore, these observations did not require custom slitmasks. Instead, the spectra were obtained through long slits. The instrument configuration for the long-slit exposures was identical to the configuration for custom multi-slit masks. To estimate the seeing, we measured the FWHM from the guider camera of the star used to the focus the telescope. All but two of the spectra of halo field stars presented here were obtained during nights of poor transparency in 2008 April and May. Star VII-18 in the GC M92 was also observed in this manner.

The raw frames were reduced into one-dimensional spectra using version 1.1.4 of spec2d, the DEIMOS data reduction pipeline developed by the DEEP Galaxy Redshift Survey. ${ }^{15}$ In addition to the procedure described by Guhathakurta et al. (2006), KGS08, and in Paper I, we tweaked the arc lamp wavelength solution by using the pipeline's optional SKYTWEAK_1D

\footnotetext{
15 http://astro.berkeley.edu/ cooper/deep/spec $2 \mathrm{~d} /$
} 


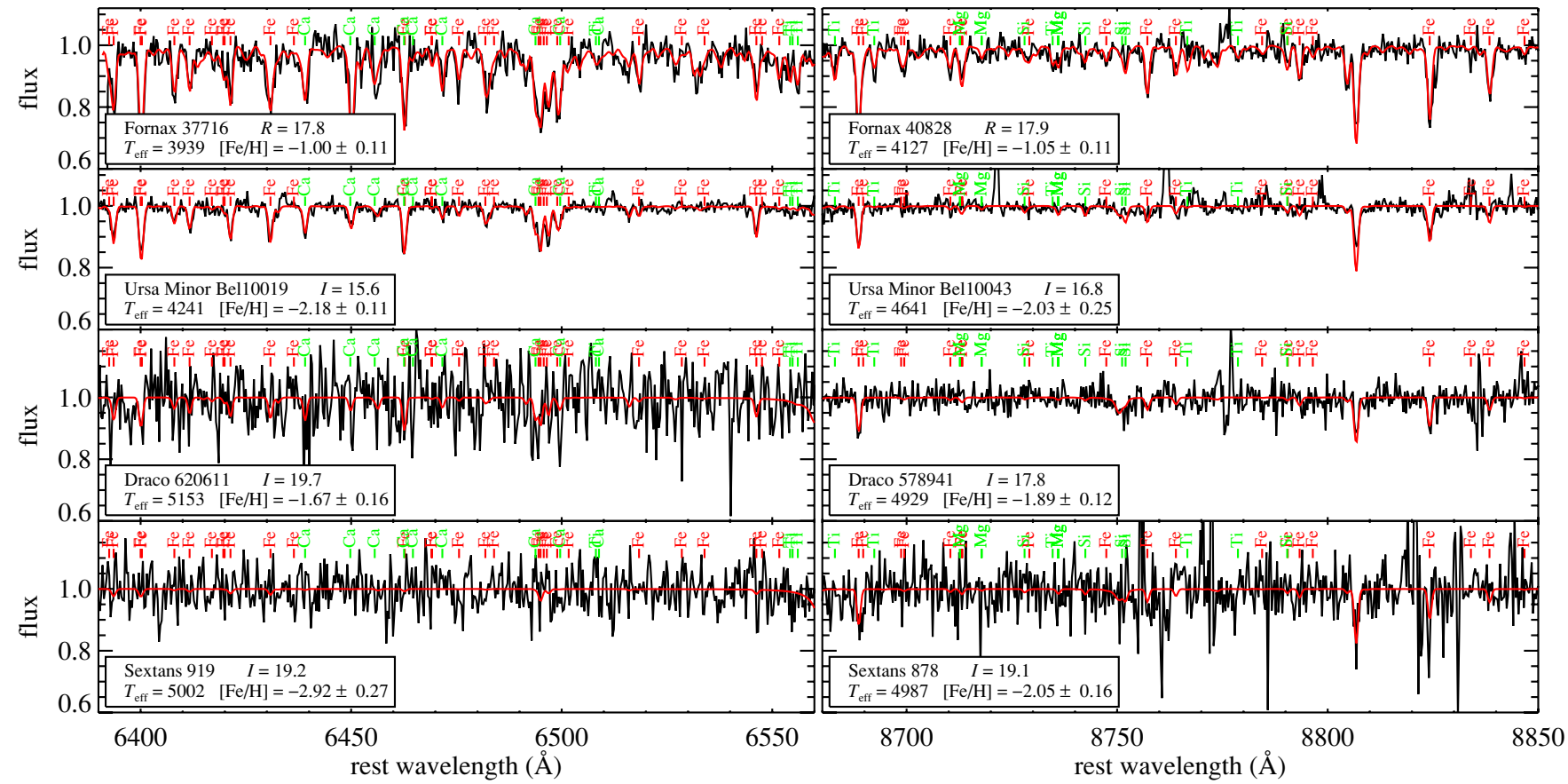

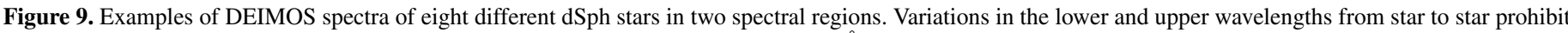

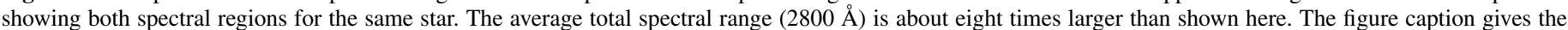

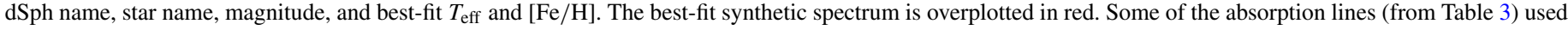
in the abundance measurements are labeled.

(A color version of this figure is available in the online journal.)

procedure. The procedure evaluates small wavelength shifts between the observed and expected wavelengths of night sky emission lines in $100 \AA$ regions. A polynomial fit to the individual shifts adjusts the wavelength array of the observed spectrum. For any given pixel, a typical wavelength shift is $0.1 \AA$.

The Fornax slitmasks for1B, for6, and for7 (see Table 2) required special attention. Because exposures for the same masks were taken months apart, the heliocentric velocity corrections changed enough that frames from the different months could not be stacked without affecting spectral line widths significantly. In order to overcome the velocity shift, we extracted one set of one-dimensional spectra for each night. The wavelength arrays for the second epoch were shifted by the difference in the heliocentric velocity correction between the two nights. The velocityshifted spectra were rebinned to match the wavelength arrays of the spectra from the first epoch. Then, the one-dimensional spectra were co-added. The rest of the analysis proceeded in the same way as for the slitmasks observed on single nights.

Figure 9 shows small spectral regions for eight different dSph stars. The stars were chosen to show a range of signalto-noise ratio $(\mathrm{S} / \mathrm{N})$ and metallicity. Some absorption lines used in the abundance determination are labeled. Some prominent lines are not labeled because they are not included in the abundance measurements. For example, $\mathrm{Mg}_{\mathrm{I}} \lambda 8807$ and its blended neighbor, Fe I $\lambda 8805$, are not labeled because the $\mathrm{Mg}$ line is too strong for a local thermodynamic equilibrium abundance measurement.

\subsection{Membership}

We determined membership in each $\mathrm{dSph}$ by the radial velocities of the stars. (Table 4 does not include radial velocities. Geha et al. will publish the radial velocities in a separate work.) In order to measure radial velocities, we cross-correlated each one-dimensional spectrum with several different stellar templates (see Simon \& Geha 2007). We adopted the velocity of the cross-correlation peak of the best-fitting template. Then, we fit a Gaussian to the velocity distribution of each dSph. Every star within $\pm 3 \sigma$ of the peak $(99.7 \%$ of the true members, assuming that they are normally distributed in velocity) was considered a member. Stars outside of this range were discarded, and they are not included in the catalog in Section 3.8. Finally, horizontal branch stars and stars obviously not belonging the red giant branch of the $\mathrm{dSph}$ were excluded. These steps reduce contamination by foreground and background MW stars, but it is impossible to eliminate contamination entirely. The small remaining contamination may influence the interpretation of the abundance measurements, but it does not our affect our estimation of their accuracy and precision (Section 4). The number of unique member stars across all eight dSphs is 2961.

\section{ABUNDANCE MEASUREMENTS}

KGS08 and Paper I discuss the details of the MRS technique used to create the catalog in this article. Here we present only a summary and some modifications to the method. Other than the changes described in Sections 3.2-3.6, the details of the procedure remain identical to the method of KGS08 with the modifications in Paper I.

\subsection{Summary of the Technique}

Each observed spectrum was compared to a large grid of synthetic spectra at a variety of effective temperatures $\left(T_{\text {eff }}\right)$, surface gravities $(\log g)$, metallicities (represented by $[\mathrm{Fe} / \mathrm{H}]$, but all elements heavier than He were modulated), and alpha enhancements $(\langle[\alpha / \mathrm{Fe}]\rangle$, an additional modulation for $\mathrm{Mg}, \mathrm{Si}$, $\mathrm{Ca}$, and $\mathrm{Ti})$. Section 3.2 gives additional details on the grid. The spectra were synthesized with the local thermodynamic 
Table 3

Important Atomic Spectral Lines

\begin{tabular}{|c|c|c|c|}
\hline Species & Wavelength $(\AA)$ & Excitation Potential (eV) & $\log (g f)$ \\
\hline $\mathrm{Mg}_{\mathrm{I}}$ & 6319.237 & 5.110 & -2.150 \\
\hline $\mathrm{Mg}_{\mathrm{I}}$ & 6319.495 & 5.108 & -2.630 \\
\hline $\mathrm{Mg}_{\mathrm{I}}$ & 7691.553 & 5.753 & -0.800 \\
\hline $\operatorname{Mg}_{\mathrm{I}}$ & 7811.133 & 5.946 & -1.550 \\
\hline $\operatorname{Mg}_{I}$ & 7811.141 & 5.946 & -1.550 \\
\hline $\mathrm{Mg}_{\mathrm{I}}$ & 8047.720 & 5.932 & -1.970 \\
\hline $\operatorname{Mg}_{I}$ & 8098.719 & 5.946 & -1.120 \\
\hline $\mathrm{Mg}_{\mathrm{I}}$ & 8098.727 & 5.946 & -1.450 \\
\hline $\mathrm{Mg}_{\mathrm{I}}$ & 8213.034 & 5.753 & -0.509 \\
\hline $\mathrm{Mg}_{\mathrm{I}}$ & 8346.106 & 5.946 & -1.080 \\
\hline
\end{tabular}

Notes. This is not a complete line list. It is a subset of Table 4 of KGS08.

(This table is available in its entirety in a machine-readable form in the online journal. A portion is shown here for guidance regarding its form and content.)

equilibrium, plane-parallel spectrum synthesis code MOOG (Sneden 1973), ATLAS9 model atmospheres (Kurucz 1993; Sbordone et al. 2004; Sbordone 2005), and atomic and molecular transition data from the Vienna Atomic Line Database (VALD; Kupka et al. 1999), modified as described by KGS08. Table 3 gives a subset of the line list for the lines visible in a high $\mathrm{S} / \mathrm{N}$ DEIMOS spectrum of a red giant with $[\mathrm{Fe} / \mathrm{H}] \sim-0.5$, such as Arcturus. A Levenberg-Marquardt algorithm (the IDL code MPFIT by Markwardt 2009) minimized $\chi^{2}$ to find the best-fitting synthetic spectrum in several steps. In the first step, $T_{\text {eff }},[\mathrm{Fe} / \mathrm{H}]$, and $\langle[\alpha / \mathrm{Fe}]\rangle$ were determined iteratively. Surface gravity was fixed by photometry. Only regions sensitive to $\mathrm{Fe}$ absorption were used to determine $\mathrm{Fe}$ and only regions sensitive to $\mathrm{Mg}, \mathrm{Si}, \mathrm{Ca}$, or $\mathrm{Ti}$ absorption were used to determine $\langle[\alpha / \mathrm{Fe}]\rangle$ (see Paper I). The observed spectrum's continuum was refined between each iteration by removing a high-order spline fit to the quotient of the observed spectrum and the best-fitting synthetic spectrum of the previous iteration. The temperature was allowed to vary, but within the bounds of a photometrically determined temperature based on available photometry. (The spectroscopic refinement of the temperature leads to more accurate abundances when compared to HRS measurements. Because lines of ionized species are sparse in these far-red spectra of red giants, spectroscopic refinement of surface gravity is not possible.) In the next steps, the individual $\mathrm{Mg}, \mathrm{Si}, \mathrm{Ca}$, and $\mathrm{Ti}$ abundances were determined by fitting the spectral regions sensitive to absorption from each element.

Special consideration was made for the MW halo field star sample. Because the distances to the halo field stars are poorly known, surface gravities could not be fixed by photometry. However, every star in the MW halo field star sample was chosen because previous authors had already observed it with HRS. We adopted the high-resolution measurements of surface gravities for all of the MW halo field stars.

\subsection{Expansion of the Spectral Grid}

In Paper I, we announced the discovery of one star in Sculptor at $[\mathrm{Fe} / \mathrm{H}]=-3.80$, which Frebel et al. (2010a) confirmed with the Magellan/MIKE high-resolution spectrograph. The lower $[\mathrm{Fe} / \mathrm{H}]$ limit of the spectral grid of KGS08 and Paper I was $[\mathrm{Fe} / \mathrm{H}]=-4.0$. If any star reached the lower $[\mathrm{Fe} / \mathrm{H}]$ limit, we discarded it under the presumption that the $\mathrm{S} / \mathrm{N}$ was inadequate to yield an $[\mathrm{Fe} / \mathrm{H}]$ measurement of useful precision. However, the confirmed existence of a star very close to the grid limit prompted us to expand the spectral grid to $[\mathrm{Fe} / \mathrm{H}]=-5.0$. Although we have not recovered any ultra metal-poor stars $(-5<[\mathrm{Fe} / \mathrm{H}]<-4)$, the expansion gives us confidence that we have not discarded any such stars because of a limitation in the grid.

Expanding the grid required computing model atmospheres at $-5<[\mathrm{Fe} / \mathrm{H}]<-4$ with $T_{\text {eff }}, \log g$, and $\langle[\alpha / \mathrm{Fe}]\rangle$ on the grid described by Table 3 of Paper I. For each value of $T_{\text {eff }}$ and $\log g$ in that table, we computed ODFNEW opacity distribution functions (Castelli \& Kurucz 2004) using R. L. Kurucz's DFSYNTHE code (described by Castelli 2005) at $[\mathrm{Fe} / \mathrm{H}]=-4.5$ and -5.0 for each of 21 steps in $\langle[\alpha / \mathrm{Fe}]\rangle$ $(0.1$ dex between $[\alpha / \mathrm{Fe}]=-1.2$ and +0.8$)$ and 2 steps in microturbulent velocity $(\xi)$. The values of $\xi$ were chosen to be the two velocities that bracket the microturbulent velocity appropriate for the star's surface gravity (Equation (2) of Paper I). The values of $[\mathrm{Fe} / \mathrm{H}]$ and $\langle[\alpha / \mathrm{Fe}]\rangle$ in the computation of the opacity distribution functions are given relative to the solar abundances of Anders \& Grevesse (1989), except that the abundance of iron is $12+\log \epsilon(\mathrm{Fe})=7.52$ (see Sneden et al. 1992, for an explanation). We computed the model

Table 4

DEIMOS Multi-element Abundances Catalog

\begin{tabular}{|c|c|c|c|c|c|c|c|c|c|}
\hline $\mathrm{dSph}$ & Name & $\begin{array}{c}T_{\text {eff }} \pm \delta_{\text {spec }} \pm \delta_{\text {phot }} \\
(\mathrm{K})\end{array}$ & $\begin{array}{c}\log g \\
\left(\mathrm{~cm} \mathrm{~s}^{-2}\right)\end{array}$ & $\begin{array}{c}\xi \\
\left(\mathrm{km} \mathrm{s}^{-1}\right)\end{array}$ & $\begin{array}{c}{[\mathrm{Fe} / \mathrm{H}]} \\
(\mathrm{dex})\end{array}$ & $\begin{array}{c}{[\mathrm{Mg} / \mathrm{Fe}]} \\
(\mathrm{dex})\end{array}$ & $\begin{array}{c}{[\mathrm{Si} / \mathrm{Fe}]} \\
(\mathrm{dex})\end{array}$ & $\begin{array}{c}{[\mathrm{Ca} / \mathrm{Fe}]} \\
(\mathrm{dex})\end{array}$ & $\begin{array}{c}{[\mathrm{Ti} / \mathrm{Fe}]} \\
(\mathrm{dex})\end{array}$ \\
\hline $\mathrm{Scl}$ & 1002473 & $5085 \pm 101 \pm 258$ & $2.02 \pm 0.08$ & 1.66 & $-2.30 \pm 0.15$ & $+0.65 \pm 0.93$ & $+0.59 \pm 0.17$ & $+0.64 \pm 0.26$ & $+0.26 \pm 0.48$ \\
\hline Scl & 1002447 & $4689 \pm 47 \pm 109$ & $1.27 \pm 0.06$ & 1.84 & $-2.04 \pm 0.12$ & $+0.36 \pm 0.28$ & $+0.47 \pm 0.12$ & $+0.33 \pm 0.16$ & $+0.28 \pm 0.09$ \\
\hline Scl & 1003386 & $4660 \pm 41 \pm 125$ & $1.49 \pm 0.06$ & 1.79 & $-1.30 \pm 0.11$ & $-0.50 \pm 0.44$ & $+0.21 \pm 0.23$ & $+0.14 \pm 0.16$ & $+0.03 \pm 0.21$ \\
\hline Scl & 1003505 & $4366 \pm 31 \pm 71$ & $0.88 \pm 0.06$ & 1.93 & $-1.82 \pm 0.11$ & $+0.26 \pm 0.19$ & $+0.20 \pm 0.14$ & $+0.20 \pm 0.14$ & $+0.00 \pm 0.10$ \\
\hline Scl & 1003443 & $4732 \pm 41 \pm 110$ & $1.26 \pm 0.06$ & 1.84 & $-1.62 \pm 0.11$ & $+0.51 \pm 0.34$ & $+0.06 \pm 0.16$ & $+0.20 \pm 0.18$ & $+0.05 \pm 0.11$ \\
\hline Scl & 1003702 & $4581 \pm 58 \pm 111$ & $1.58 \pm 0.06$ & 1.77 & $-1.89 \pm 0.12$ & $\cdots$ & $+0.34 \pm 0.36$ & $+0.10 \pm 0.14$ & $+0.11 \pm 0.17$ \\
\hline Scl & 1003967 & $4525 \pm 58 \pm 86$ & $1.11 \pm 0.05$ & 1.88 & $-2.66 \pm 0.13$ & $\cdots$ & $+0.28 \pm 0.82$ & $+0.60 \pm 0.22$ & $+0.40 \pm 0.23$ \\
\hline
\end{tabular}

Notes. Some columns (right ascension, declination, and $B, V, R$, and $I$ magnitudes) are suppressed in the printed version.

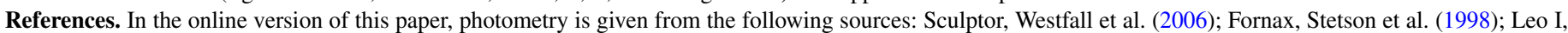

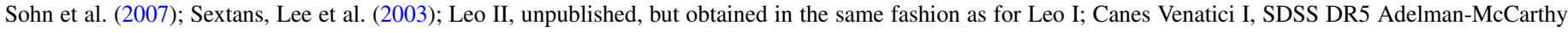
et al. (2007); Ursa Minor, Bellazzini et al. (2002); and Draco, Ségall et al. (2007).

(This table is available in its entirety in a machine-readable form in the online journal. A portion is shown here for guidance regarding its form and content.) 
atmospheres with Kurucz's (1993) ATLAS9 code ported into Linux (Sbordone et al. 2004; Sbordone 2005). Convective overshooting was turned off, and the mixing length parameter for convection was $l / H_{p}=1.25$. These convection parameters are the same as for Castelli \& Kurucz's (2004) grid of ATLAS9 atmospheres. We linearly interpolated the atmospheres in the $[\mathrm{Fe} / \mathrm{H}]$ and $\xi$ dimensions to populate the grid described in Paper I.

\subsection{Correction to Continuum Division}

Following Shetrone et al. (2009), we modified the method of KGS08 to perform better continuum determination in Paper I. We iteratively determined the continuum by fitting a high-order spline to the quotient of the observed spectrum and the bestfitting synthetic spectrum of the previous iteration. In Paper I, we incorporated this technique into the Levenberg-Marquardt algorithm that determines the best-fitting synthetic spectrum. During each Levenberg-Marquardt iteration, any remaining fluctuations in the continuum level were removed before the $\chi^{2}$ between the observed spectrum and a trial synthetic spectrum was evaluated. Instead, we decided that it was more appropriate to determine the best-fitting synthetic spectrum without modifying the observed spectrum in the minimization of $\chi^{2}$. The continuum refinement occurred between separate Levenberg-Marquardt determinations of the best-fitting synthetic spectrum. The continuum refinement was considered converged when $[\mathrm{Fe} / \mathrm{H}]$ and the bulk $\langle[\alpha / \mathrm{Fe}]\rangle$ changed by less than $0.001 \mathrm{dex}$ and $T_{\mathrm{eff}}$ changed by less than $1 \mathrm{~K}$.

\subsection{Sigma Clipping}

After the continuum refinement, we added a new step not used by KGS08 or in Paper I: masking pixels whose absolute difference from the best-fit synthetic spectrum exceeded 2.5 times their variance. The remaining steps to measure $[\mathrm{Fe} / \mathrm{H}]$ were performed on the clipped spectrum. The sigma clipping excludes spectral regions with artifacts, such as improperly removed cosmic rays. It also insulates the abundance measurements from $\mathrm{Fe}$ absorption lines with incorrect oscillator strengths. The comparison between MRS and HRS abundances (Section 4.4) improved slightly as a result of including sigma clipping. We experimented with the threshold value, and we found that $2.5 \sigma$ included $\gtrsim 90 \%$ of the pixels while reducing the mean and standard deviations of the differences between MRS and HRS abundances.

\subsection{New Approach for $[\alpha / \mathrm{Fe}]$}

Previously (Paper I), we measured [Mg/Fe], [Si/Fe], $[\mathrm{Ca} / \mathrm{Fe}]$, and $[\mathrm{Ti} / \mathrm{Fe}]$ by searching the spectral grid at fixed, previously determined $T_{\text {eff }}$ and $[\mathrm{Fe} / \mathrm{H}]$. Because each element was not a separate dimension, abundances of all of the $\alpha$ elements $(\mathrm{O}, \mathrm{Ne}, \mathrm{Mg}, \mathrm{Si}, \mathrm{S}, \mathrm{Ar}, \mathrm{Ca}$, and $\mathrm{Ti})$ varied together. Individual elements were measured by isolating the spectral regions containing those lines. This resulted in a different value of $\langle[\alpha / \mathrm{Fe}]\rangle$ for the model atmospheres used in the measurement of each element. However, the parameter $\langle[\alpha / \mathrm{Fe}]\rangle$ controls not only the strength of $\alpha$ element lines, but also important atmospheric quantities, such as the free electron fraction. The resulting changes in the continuous opacity affect absorption lines of all elements. For red giants, increasing the atmospheric value of $\langle[\alpha / \mathrm{Fe}]\rangle$ at fixed $[\mathrm{Fe} / \mathrm{H}]$ raises the continuous opacity in the red spectral region by increasing the number density of $\mathrm{H}^{-}$ions. Metal lines become weaker.
In the present catalog, we have determined the individual $\alpha$ element ratios by fixing the atmospheric value of $\langle[\alpha / \mathrm{Fe}]\rangle$, which we call $[\alpha / \mathrm{Fe}]_{\mathrm{atm}}$. We synthesized a subgrid of spectra with an additional dimension, $[\alpha / \mathrm{Fe}]_{\text {abund }}$. The new dimension is the $\langle[\alpha / \mathrm{Fe}]\rangle$ ratio for the abundances of $\mathrm{Mg}, \mathrm{Si}, \mathrm{Ca}$, and Ti at fixed $[\alpha / \mathrm{Fe}]_{\mathrm{atm}}$. Like $[\alpha / \mathrm{Fe}]_{\mathrm{atm}},[\alpha / \mathrm{Fe}]_{\mathrm{abund}}$ is spaced at 0.1 dex from -0.8 to +1.2 . It was not necessary to synthesize spectra with $[\alpha / \mathrm{Fe}]_{\mathrm{atm}}=[\alpha / \mathrm{Fe}]_{\text {abund }}$ because the existing grid already contained such spectra. To save computation time, the new subgrid included only the spectral regions used in the measurement of $\mathrm{Mg}, \mathrm{Si}, \mathrm{Ca}$, and $\mathrm{Ti}$.

We determined $[\alpha / \mathrm{Fe}]_{\mathrm{atm}}$ by fitting to the $\mathrm{Mg}, \mathrm{Si}, \mathrm{Ca}$, and Ti lines simultaneously. Then, the values of $[\mathrm{Mg} / \mathrm{Fe}],[\mathrm{Si} / \mathrm{Fe}]$, $[\mathrm{Ca} / \mathrm{Fe}]$, and $[\mathrm{Ti} / \mathrm{Fe}]$ were determined by variation of $[\alpha / \mathrm{Fe}]_{\text {abund }}$ with $T_{\text {eff }}, \log g,[\mathrm{Fe} / \mathrm{H}]$, and $[\alpha / \mathrm{Fe}]_{\text {atm }}$ held fixed.

The complete list of steps to determine the abundances is largely the same as in Section 4.7 of Paper I. The following is the updated list.

1. $T_{\text {eff }}$ and $[\mathrm{Fe} / \mathrm{H}]$, first pass: an observed spectrum was compared to a synthetic spectrum with $T_{\text {eff }}$ and $\log g$ determined photometrically. Only spectral regions most susceptible to Fe absorption were considered. $T_{\text {eff }}$ was loosely constrained by photometry. Photometry alone determined $\log g$.

2. $[\alpha / \mathrm{Fe}]_{\text {atm }}$, first pass: $T_{\text {eff }}, \log g$, and $[\mathrm{Fe} / \mathrm{H}]$ were fixed, but $[\alpha / \mathrm{Fe}]_{\mathrm{atm}}$ and $[\alpha / \mathrm{Fe}]_{\mathrm{abund}}$ varied together. Only the spectral regions susceptible to absorption by $\mathrm{Mg}, \mathrm{Si}, \mathrm{Ca}$, or Ti were considered.

3. Continuum refinement: the continuum-divided, observed spectrum was divided by the synthetic spectrum with the parameters determined in steps 1 and 2 . The result approximated a flat noise spectrum. To better determine the continuum, we fit a B-spline with a breakpoint spacing of 150 pixels to the residual spectrum. We divided the observed spectrum by the spline fit.

4. Steps 1-3 were repeated until $T_{\text {eff }}$ changed from the previous iteration by less than $1 \mathrm{~K}$ and $[\mathrm{Fe} / \mathrm{H}]$ changed by less than 0.001 dex.

5. $[\mathrm{Fe} / \mathrm{H}]$, second pass: we repeated step 1 with the revised, sigma-clipped spectrum, but $T_{\text {eff }}$ was held fixed at the previously determined value.

6. $[\alpha / \mathrm{Fe}]_{\mathrm{atm}}$, second pass: we repeated step 2 with the revised spectrum and the value of $[\mathrm{Fe} / \mathrm{H}]$ from step 5 .

7. $[\mathrm{Fe} / \mathrm{H}]$, third pass: we repeated step 5 with the value of $[\alpha / \mathrm{Fe}]_{\mathrm{atm}}$ from step 6 .

8. $[\mathrm{Mg} / \mathrm{Fe}]$ : we determined $[\mathrm{Mg} / \mathrm{Fe}]$ by varying $[\alpha / \mathrm{Fe}]_{\text {abund }}$ at fixed $[\alpha / \mathrm{Fe}]_{\mathrm{atm}}$. Only spectral regions subject to $\mathrm{Mg}$ absorption were considered.

9. [Si/Fe]: we repeated step 9 for $\mathrm{Si}$ instead of $\mathrm{Mg}$.

10. $[\mathrm{Ca} / \mathrm{Fe}]$ : we repeated step 9 for $\mathrm{Ca}$ instead of $\mathrm{Mg}$.

11. $[\mathrm{Ti} / \mathrm{Fe}]$ : we repeated step 9 for $\mathrm{Ti}$ instead of $\mathrm{Mg}$.

\subsection{Offsets to the Abundance Measurements}

In Paper I, we found that adding 0.15 dex to the MRS measurement of $[\mathrm{Fe} / \mathrm{H}]$ was necessary to bring $[\mathrm{Fe} / \mathrm{H}]_{\mathrm{MRS}}$ into agreement with $[\mathrm{Fe} / \mathrm{H}]_{\mathrm{HRS}}$ for nine stars with both MRS and HRS measurements in Sculptor. The modification to the continuum division and sigma clipping mitigated the need for the artificial offset in $[\mathrm{Fe} / \mathrm{H}]$. There is still an offset of -0.07 dex, but it is small enough that we do not correct it.

An offset of 0.15 dex between $[\mathrm{Si} / \mathrm{Fe}]_{\mathrm{MRS}}$ and $[\mathrm{Si} / \mathrm{Fe}]_{\mathrm{HRS}}$ still exists, and we added 0.15 dex to $[\mathrm{Si} / \mathrm{Fe}]_{\mathrm{MRS}}$ for better agreement. This offset is unique to $\mathrm{Si}$, and does not affect 
Table 5

Abundance Error Floors

\begin{tabular}{lc}
\hline \hline Element Ratio & $\delta_{\text {sys }}$ \\
\hline$[\mathrm{Fe} / \mathrm{H}]$ & 0.113 \\
{$[\mathrm{Mg} / \mathrm{Fe}]$} & 0.095 \\
{$[\mathrm{Si} / \mathrm{Fe}]$} & 0.104 \\
{$[\mathrm{Ca} / \mathrm{Fe}]$} & 0.118 \\
{$[\mathrm{Ti} / \mathrm{Fe}]$} & 0.083 \\
\hline
\end{tabular}

other $\langle[\alpha / \mathrm{Fe}]\rangle$ ratios. Therefore, the offset does not arise from continuum division uncertainties. We share eight Si I lines in common with the high-resolution studies of Cohen et al. (e.g., Cohen \& Huang 2009, 2010). Our oscillator strengths for these eight lines are all larger, with an average difference of 0.16 dex. Therefore, our Si measurements are lower than Cohen et al.'s Si measurements. Rather than adjust the oscillator strengths and recompute the spectral grid at large computational expense, we added 0.15 dex to our $[\mathrm{Si} / \mathrm{Fe}]$ measurements.

\subsection{Applicability to Dwarf Stars}

The maximum $\log g$ in the dSph catalog is 3.5. Some of the GC masks observed in 2009 (see Table 2) included stars below the main sequence turnoff. We excluded GC and MW halo stars with $\log g>3.5$ in order to restrict the comparison sample to stars of the same spectral type as the dSph catalog.

\subsection{The Catalog}

Table 4 gives the measurements of 2961 unique stars (376 in Sculptor, 675 in Fornax, 827 in Leo I, 141 in Sextans, 258 in Leo II, 174 in Canes Venatici I, 212 in Ursa Minor, and 298 in Draco). The table lists the right ascension, declination, extinction-corrected $B V R I$ magnitudes where available (sometimes converted from ugriz following Jordi et al. 2006), temperature, surface gravity, microturbulent velocity, $[\mathrm{Fe} / \mathrm{H}]$, and the $[\mathrm{Mg} / \mathrm{Fe}],[\mathrm{Si} / \mathrm{Fe}],[\mathrm{Ca} / \mathrm{Fe}]$, and $[\mathrm{Ti} / \mathrm{Fe}]$ ratios where measurable. Uncertainties are also given for all of the measurable quantities except the space coordinates and microturbulent velocity. For $T_{\text {eff }}$, two types of uncertainty are given. The first type, $\delta T_{\text {eff,spec }}$, is the uncertainty from random spectral noise. (In detail, it is the square root of the diagonal element of the covariance matrix corresponding to $\delta T_{\text {eff }}$ when both $T_{\text {eff }}$ and $[\mathrm{Fe} / \mathrm{H}]$ are allowed to vary.) The second type, $\delta T_{\text {eff,phot }}$, is the uncertainty on the photometric estimate of $T_{\text {eff }}$, accounting for random photometric uncertainty and systematic error from isochrone modeling (Equation (6) of Paper I). Because $\log g$ is determined from photometry alone, the uncertainty on $\log g$ is derived analogously to $\delta T_{\text {eff,phot }}$, but the error on the distance modulus to each stellar system is included in the error estimate of $\log g$ as well. The uncertainties on the abundance measurements are the $1 \sigma$ uncertainties from the spectral fit added in quadrature with the constant error floor, given in Table 5.

As an example, consider the first star in Table 4. The first column identifies its origin as Sculptor, and the second column gives the star's identification number. In the online version, the next two columns give its coordinates. $V$ and $I$ magnitudes are given, but $B$ and $R$ magnitudes are not available. We have measured $T_{\text {eff }}=5085 \mathrm{~K}$ with an uncertainty of $101 \mathrm{~K}$ based on spectral noise. From photometry alone, the uncertainty is $258 \mathrm{~K}$. We have also measured a photometric surface gravity of $\log g=2.02 \pm 0.08$. The microturbulent velocity, deduced solely from the surface gravity, is $1.66 \mathrm{~km} \mathrm{~s}^{-1}$. The remaining columns give $[\mathrm{Fe} / \mathrm{H}],[\mathrm{Mg} / \mathrm{Fe}],[\mathrm{Si} / \mathrm{Fe}],[\mathrm{Ca} / \mathrm{Fe}]$, and $[\mathrm{Ti} / \mathrm{Fe}]$ and their estimated uncertainties.

\section{ACCURACY}

The range of abundances and size of our sample demand rigorous checks to validate the accuracy and precision of our measurements. In this section, we estimate measurement uncertainties, check those estimates against repeat measurements of the same stars, and check the accuracy of our measurements against previously published high-resolution spectroscopic abundance measurements.

\subsection{Estimation of Measurement Uncertainty}

We estimated abundance measurement uncertainties from individual stars in GCs. Because we have excluded GCs suspected to exhibit Fe abundance variations, such as M22 (Da Costa et al. 2009; Marino et al. 2009), we assumed that the spread of $[\mathrm{Fe} / \mathrm{H}]$ in each cluster is much smaller than the measurement uncertainty, but we made no such assumption for other elements. Although no monometallic cluster except NGC 2419 (Cohen et al. 2010) has been found to have a measurable spread of Ca or Ti abundances, many clusters exhibit star-to-star $\mathrm{Mg}$ variations (see the review by Gratton et al. 2004).

KGS08 and Paper I explicitly described the procedure for determining measurement uncertainties. For each elemental abundance measurement, they added an extra error component in quadrature with the random error from spectral noise. This error floor for $[\mathrm{Fe} / \mathrm{H}]$ was determined from the excess spread in the measured $[\mathrm{Fe} / \mathrm{H}]$ in each $\mathrm{GC}$ after accounting for random error due to spectral noise. For this purpose, we excluded M92 because we observed only one star in it, and we excluded NGC 2419 because we suspect that it may contain a spread in heavy element abundances (Cohen et al. 2010). The error floor in the four $\langle[\alpha / \mathrm{Fe}]\rangle$ ratios was determined from comparison to high-resolution measurements. NGC 2419 was included for this purpose. The error floor is the value needed to account for differences between the MRS and HRS measurements after removing the MRS random error and the published value of the HRS measurement uncertainty. Table 5 gives the updated values for the error floors for each element. The differences between this table and Table 5 of Paper I arise from the modifications described in Section 3 and the inclusion of GC observations obtained since the publication of KGS08.

\subsection{Errors from Atmospheric Parameters}

Errors in $T_{\text {eff }}, \log g$, and $\xi$ have the potential to change the measured abundances significantly. The error floor for $[\mathrm{Fe} / \mathrm{H}]_{\text {MRS }}$ accounts for uncertainty in the atmospheric parameters, but we also quantify the response of $[\mathrm{Fe} / \mathrm{H}]$ to forced changes in $T_{\text {eff }}$ and $\log g$. Differences in $T_{\text {eff }}, \log g$, and $\xi$ affect the $\alpha$ element abundance ratios less than they affect $[\mathrm{Fe} / \mathrm{H}]$. To first order, the measurement of an $\alpha$ element's abundance responds to the atmospheric parameters in the same way as the measurement of the $\mathrm{Fe}$ abundance. As a result, the division of one element's abundance by the Fe abundance somewhat masks errors in atmospheric parameters.

We recomputed abundances at $125 \mathrm{~K}$ and $250 \mathrm{~K}$ above and below the best-fitting $T_{\text {eff }}$ for every star in the GC, MW halo, and $\mathrm{dSph}$ samples. The new abundance was determined by searching the grid for the synthetic spectrum that best matched the observed spectrum with $T_{\text {eff }}$ and $\log g$ held fixed. We also recomputed abundances with $\log g$ held fixed at 0.3 dex and 
0.6 dex above and below the best-fitting $\log g$. The measured abundance of $[\mathrm{Fe} / \mathrm{H}]$ is higher for a larger value of $T_{\text {eff }}$ or a larger value of $\log g$. Conversely, the measured abundance of $[\mathrm{Fe} / \mathrm{H}]$ is lower for a lower value of $T_{\text {eff }}$ or a lower value of $\log g$. (See Section 6.3 of KGS08 for a more thorough discussion.)

The GC star sample provides some insight on the general response of $[\mathrm{Fe} / \mathrm{H}]$ to changes in atmospheric parameters. The average change in $[\mathrm{Fe} / \mathrm{H}]$ is \pm 0.092 dex per $\pm 100 \mathrm{~K}$ change in $T_{\text {eff. }}$. The average change in $[\mathrm{Fe} / \mathrm{H}]$ is \pm 0.039 dex per \pm 1 dex change in $\log g$. The $[\mathrm{Fe} / \mathrm{H}]$ measurements are relatively insensitive to changes in $\log g$ because the abundance analysis uses many Fe I lines but virtually no Fe II lines, which are few and weak in red giants. Fe I lines vary little with $\log g$. Ideally, we would use only Fe II lines because they are not subject to overionization problems (Thévenin \& Idiart 1999; Ivans et al. 2001). However, we must use Fe I lines because Fe II lines are not visible in DEIMOS spectra of red giants. The slopes of $\Delta[\mathrm{Fe} / \mathrm{H}] / \Delta T_{\text {eff }}$ and $\Delta[\mathrm{Fe} / \mathrm{H}] / \Delta(\log g)$ are approximately linear within $\pm 250 \mathrm{~K}$ and \pm 0.6 dex.

Table 6 gives the response of $[\mathrm{Fe} / \mathrm{H}],[\mathrm{Mg} / \mathrm{Fe}],[\mathrm{Si} / \mathrm{Fe}]$, $[\mathrm{Ca} / \mathrm{Fe}]$, and $[\mathrm{Ti} / \mathrm{Fe}]$ to a change of $125 \mathrm{~K}$ and $250 \mathrm{~K}$ in $T_{\text {eff }}$ and $0.3 \mathrm{dex}$ and $0.6 \mathrm{dex}$ in $\log g$. The values given are the averages of the absolute values of the increase and decrease in $T_{\text {eff }}$ or $\log g$. For example, consider a star with $[\mathrm{Fe} / \mathrm{H}]=-2.00$ and $T_{\text {eff }}=4500 \mathrm{~K}$. If the column for $\Delta[\mathrm{Fe} / \mathrm{H}](125 \mathrm{~K})$ lists 0.12 , then it can be assumed that the measured abundance of the star would be $[\mathrm{Fe} / \mathrm{H}]=-1.88$ if $T_{\text {eff }}$ were truly $4625 \mathrm{~K}$, and the measured abundance would be $[\mathrm{Fe} / \mathrm{H}]=-2.12$ if $T_{\text {eff }}$ were truly $4375 \mathrm{~K}$.

We could not estimate the effect of errors in microturbulent velocity as easily as for $T_{\text {eff }}$ and $\log g$ because our grid of spectra did not have a separate dimension for $\xi$. Instead, we fixed $\xi$ based on a calibration to $\log g$ (Equation (2) of Paper I). To investigate the effect of errors in $\xi$ on $[\mathrm{Fe} / \mathrm{H}]$, we reanalyzed 20 stars from four GCs at different metallicities (M71, M5, M13, and M15). We chose the stars with $\log g$ nearest to $0.5,1.0,1.5,2.0,2.5$, and 3.0, where available. We synthesized a new, small grid of spectra for each star. We generated new atmospheres by linear interpolation in our ATLAS9 grid (Section 3.2). The values of $T_{\text {eff }}$ and $\log g$ were chosen to be the best-fit values we already determined for each star. Values of $[\mathrm{Fe} / \mathrm{H}]$ were spaced at every 0.1 dex from at least 0.4 dex below to at least 0.4 dex above the best-fit value. Values of $\xi$ were \pm 0.2 and $\pm 0.4 \mathrm{~km} \mathrm{~s}^{-1}$ around the value of $\xi$ from the surface gravity calibration. We computed spectra with MOOG. For each of the four displacements of $\xi$, we found the value of $[\mathrm{Fe} / \mathrm{H}]$ that minimized $\chi^{2}$ between the new grid of spectra and the observed spectrum. As before, we used a Levenberg-Marquardt minimization algorithm with linear interpolation to compute $\chi^{2}$ between $[\mathrm{Fe} / \mathrm{H}]$ grid points.

Figure 10 shows the changes in the measurement of $[\mathrm{Fe} / \mathrm{H}]$ induced by changes in $\xi$ for each of the $20 \mathrm{GC}$ stars. Reducing $\xi$ increases $[\mathrm{Fe} / \mathrm{H}]$ because the absorption lines saturate at lower abundance. A larger abundance is necessary to compensate for the weaker lines. The abundance change is most severe for stars of low surface gravity (low temperature) and high metallicity because those stars have strong lines and are most susceptible to line saturation. The absolute change in $[\mathrm{Fe} / \mathrm{H}]$ does not exceed $0.1 \mathrm{dex}$, even for changes of $\pm 0.4 \mathrm{~km} \mathrm{~s}^{-1}$. This result agrees with our later assessment of the effect of microturbulent velocity error (Figure 21), where we deduce a slope of $\Delta[\mathrm{Fe} / \mathrm{H}] / \Delta \xi=$ $-0.20 \mathrm{dex}\left(\mathrm{km} \mathrm{s}^{-1}\right)^{-1}$. For comparison, the standard deviation between $\xi_{\text {MRS }}$ and $\xi_{\text {HRS }}$ is $0.3 \mathrm{~km} \mathrm{~s}^{-1}$ (see Section 4.4 and Table 10).

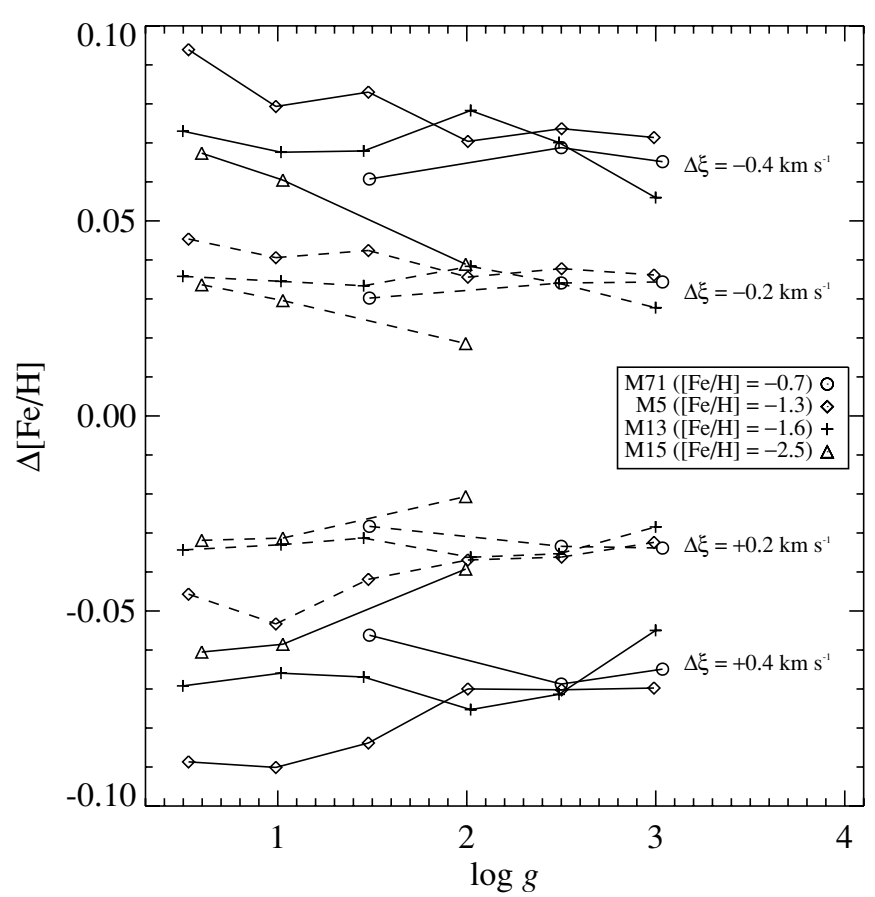

Figure 10. Differences in $[\mathrm{Fe} / \mathrm{H}]$ from changes in microturbulent velocity $(\xi)$ of $\pm 0.2 \mathrm{~km} \mathrm{~s}^{-1}$ (dashed lines) and $\pm 0.4 \mathrm{~km} \mathrm{~s}^{-1}$ (solid lines) vs. surface gravity. The 20 stars analyzed are selected from four different globular clusters over a range of metallicities and from a range of surface gravities. The figure legend gives the mean $[\mathrm{Fe} / \mathrm{H}]$ for each $\mathrm{GC}$ from our own medium-resolution measurements.

\subsection{Duplicate Observations}

The repeat observations of $167 \mathrm{dSphs}$ stars provided insight into the effect of random error on the measurements of $[\mathrm{Fe} / \mathrm{H}]$ and $\langle[\alpha / \mathrm{Fe}]\rangle$ (an average of $[\mathrm{Mg} / \mathrm{Fe}],[\mathrm{Si} / \mathrm{Fe}],[\mathrm{Ca} / \mathrm{Fe}]$, and $[\mathrm{Ti} / \mathrm{Fe}])$. Repeat measurements of $\langle[\alpha / \mathrm{Fe}]\rangle$ were possible for both stars in 141 pairs. Figures 11 and 12 summarize the comparisons of measurements of different spectra of the same stars. They show the distributions of the absolute difference between the measured $[\mathrm{Fe} / \mathrm{H}]$ and $\langle[\alpha / \mathrm{Fe}]\rangle$ for each pair of spectra divided by the expected error of the difference. In calculating the expected error of the difference, we apply the error floor to only one of the two stars. Even though the same technique is used to measure abundances in both stars-including the same photometric estimate of surface gravity - the extra error, which accounts for sources of error beyond random spectral noise, is appropriate because the wavelength range within a pair of spectra differs by up to $400 \AA$. The different $\mathrm{Fe}$ lines in these ranges span a different range of excitation potentials, and the Levenberg-Marquardt algorithm converges on different solutions.

Figure 11 also shows the best-fit Gaussian $(\sigma=0.98)$ and a Gaussian with unit variance. The best-fit Gaussian in Figure 12 has $\sigma=1.00$. The areas of the Gaussians are normalized to the number of stars. Our estimate of uncertainty is accurate because the variances of the best-fit Gaussians are close to unity.

\subsection{Comparison to High-resolution Measurements}

The most reliable test of the MRS atmospheric parameter and abundance estimates is to compare with completely independent high-resolution observations and analyses of the same stars. Table 7 lists the names, references, and coordinates of stars with previously published HRS measurements that we have also 


\begin{tabular}{|c|c|c|c|c|c|c|c|c|c|c|c|c|c|c|c|c|c|c|c|c|c|}
\hline \multirow[t]{3}{*}{$\mathrm{dSph}$} & \multirow[t]{3}{*}{ Name } & \multicolumn{4}{|c|}{$\delta[\mathrm{Fe} / \mathrm{H}]$} & \multicolumn{4}{|c|}{$\delta[\mathrm{Mg} / \mathrm{Fe}]$} & \multicolumn{4}{|c|}{$\delta[\mathrm{Si} / \mathrm{Fe}]$} & \multicolumn{4}{|c|}{$\delta[\mathrm{Ca} / \mathrm{Fe}]$} & \multicolumn{4}{|c|}{$\delta[\mathrm{Ti} / \mathrm{Fe}]$} \\
\hline & & \multicolumn{2}{|c|}{$T_{\text {eff }} \pm$} & \multicolumn{2}{|c|}{$\log g \pm$} & \multicolumn{2}{|c|}{$T_{\text {eff }} \pm$} & \multicolumn{2}{|c|}{$\log g \pm$} & \multicolumn{2}{|c|}{$T_{\text {eff }} \pm$} & \multicolumn{2}{|c|}{$\log g \pm$} & \multicolumn{2}{|c|}{$T_{\text {eff }} \pm$} & \multicolumn{2}{|c|}{$\log g \pm$} & \multicolumn{2}{|c|}{$T_{\text {eff }} \pm$} & \multicolumn{2}{|c|}{$\log g \pm$} \\
\hline & & $125 \mathrm{~K}$ & $250 \mathrm{~K}$ & 0.3 & 0.6 & $125 \mathrm{~K}$ & $250 \mathrm{~K}$ & 0.3 & 0.6 & $125 \mathrm{~K}$ & $250 \mathrm{~K}$ & 0.3 & 0.6 & $125 \mathrm{~K}$ & $250 \mathrm{~K}$ & 0.3 & 0.6 & $125 \mathrm{~K}$ & $250 \mathrm{~K}$ & 0.3 & 0.6 \\
\hline$\overline{\mathrm{Scl}}$ & 1002473 & 0.10 & 0.20 & 0.00 & 0.00 & 0.02 & 0.04 & 0.03 & 0.06 & 0.06 & 0.16 & 0.00 & 0.03 & 0.06 & 0.11 & 0.03 & 0.06 & 0.05 & 0.08 & 0.02 & 0.03 \\
\hline $\mathrm{Scl}$ & 1002447 & 0.13 & 0.26 & 0.00 & 0.00 & 0.09 & 0.12 & 0.02 & 0.03 & 0.07 & 0.16 & 0.00 & 0.01 & 0.07 & 0.12 & 0.02 & 0.03 & 0.02 & 0.08 & 0.04 & 0.04 \\
\hline $\mathrm{Scl}$ & 1002888 & 0.13 & 0.24 & 0.00 & 0.00 & 0.01 & 0.01 & 0.04 & 0.06 & 0.09 & 0.17 & 0.01 & 0.00 & 0.05 & 0.09 & 0.02 & 0.03 & 0.03 & 0.06 & 0.01 & 0.01 \\
\hline Scl & 1003386 & 0.13 & 0.26 & 0.02 & 0.04 & 0.03 & 0.04 & 0.02 & 0.05 & 0.08 & 0.19 & 0.04 & 0.03 & 0.04 & 0.09 & 0.01 & 0.04 & 0.06 & 0.11 & 0.04 & 0.04 \\
\hline Scl & 1003505 & 0.15 & 0.29 & 0.01 & 0.01 & 0.09 & 0.16 & 0.01 & 0.02 & 0.11 & 0.21 & 0.02 & 0.03 & 0.07 & 0.11 & 0.01 & 0.03 & 0.08 & 0.18 & 0.00 & 0.00 \\
\hline Scl & 1003443 & 0.13 & 0.25 & 0.01 & 0.02 & 0.11 & 0.11 & 0.01 & 0.01 & 0.08 & 0.14 & 0.01 & 0.02 & 0.03 & 0.05 & 0.01 & 0.02 & 0.03 & 0.06 & 0.01 & 0.02 \\
\hline Scl & 1003537 & 0.16 & 0.31 & 0.01 & $\ldots$ & 0.08 & 0.13 & 0.02 & 0.02 & 0.12 & 0.24 & 0.01 & 0.02 & 0.06 & 0.12 & 0.01 & 0.02 & 0.08 & 0.16 & 0.00 & 0.01 \\
\hline Scl & 1003694 & 0.09 & 0.16 & 0.07 & $\ldots$ & 0.02 & 0.01 & 0.05 & 0.06 & 0.12 & 0.23 & 0.02 & 0.01 & 0.09 & 0.12 & 0.06 & 0.08 & 0.20 & 0.29 & 0.02 & 0.06 \\
\hline Scl & 1003702 & 0.14 & 0.27 & 0.01 & 0.02 & 0.00 & 0.00 & 0.00 & 0.00 & 0.08 & 0.16 & 0.00 & 0.01 & 0.05 & 0.06 & 0.00 & 0.00 & 0.04 & 0.10 & 0.05 & 0.03 \\
\hline Scl & 1003967 & 0.14 & 0.27 & 0.01 & 0.02 & 0.00 & 0.00 & 0.00 & 0.00 & 0.08 & 0.15 & 0.01 & 0.02 & 0.04 & 0.08 & 0.01 & 0.01 & 0.01 & 0.08 & 0.01 & 0.02 \\
\hline
\end{tabular}

(This table is available in its entirety in a machine-readable form in the online journal. A portion is shown here for guidance regarding its form and content.)

Table 7

Comparison Between High-resolution and DEIMOS Abundances

\begin{tabular}{|c|c|c|c|c|c|c|c|c|c|c|c|c|}
\hline \multirow[t]{2}{*}{ System } & \multirow[t]{2}{*}{ Name } & \multirow[t]{2}{*}{ HRS Reference } & \multicolumn{5}{|c|}{ HRS } & \multicolumn{5}{|c|}{ MRS } \\
\hline & & & $\begin{array}{l}T_{\text {eff }} \\
(\mathrm{K})\end{array}$ & $\begin{array}{c}\log g \\
\left(\mathrm{~cm} \mathrm{~s}^{-2}\right)\end{array}$ & $\begin{array}{c}\xi \\
\left(\mathrm{km} \mathrm{s}^{-1}\right)\end{array}$ & $\begin{array}{c}{[\mathrm{Fe} / \mathrm{H}]} \\
(\mathrm{dex})\end{array}$ & $\begin{array}{c}{[\mathrm{Mg} / \mathrm{Fe}]} \\
(\mathrm{dex})\end{array}$ & $\begin{array}{l}T_{\text {eff }} \\
(\mathrm{K})\end{array}$ & $\begin{array}{c}\log g \\
\left(\mathrm{~cm} \mathrm{~s}^{-2}\right)\end{array}$ & $\begin{array}{c}\xi \\
\left(\mathrm{km} \mathrm{s}^{-1}\right)\end{array}$ & $\begin{array}{c}{[\mathrm{Fe} / \mathrm{H}]} \\
(\mathrm{dex})\end{array}$ & $\begin{array}{c}{[\mathrm{Mg} / \mathrm{Fe}]} \\
(\mathrm{dex})\end{array}$ \\
\hline M79 & N1904-S80 & Gratton \& Ortolani (1989) & 4250 & 0.75 & 2.50 & $-1.28 \pm 0.20$ & $+0.05 \pm 0.20$ & 4055 & 0.56 & 2.01 & $-1.56 \pm 0.11$ & $+0.07 \pm 0.11$ \\
\hline NGC 2419 & N2419-S1305 & Shetrone et al. (2001) & 4275 & 0.70 & 2.10 & $-2.32 \pm 0.11$ & $+0.30 \pm 0.18$ & 4395 & 0.79 & 1.96 & $-2.20 \pm 0.11$ & $+0.58 \pm 0.20$ \\
\hline NGC 2419 & N2419-S1305 & Shetrone et al. (2001) & 4275 & 0.70 & 2.10 & $-2.32 \pm 0.11$ & $+0.30 \pm 0.18$ & 4366 & 0.79 & 1.96 & $-2.17 \pm 0.11$ & $+0.17 \pm 0.24$ \\
\hline M5 & III-149 & Ivans et al. (2001) & 4200 & 0.91 & 1.75 & $-1.15 \pm 0.04$ & $\ldots$ & 4218 & 1.05 & 1.89 & $-1.30 \pm 0.11$ & $+0.17 \pm 0.11$ \\
\hline M5 & G18155_0228 & Ramírez \& Cohen (2003) & 5270 & 3.25 & 1.44 & $-1.31 \pm 0.04$ & $+0.28 \pm 0.06$ & 5286 & 3.25 & 1.37 & $-1.44 \pm 0.11$ & $+0.26 \pm 0.14$ \\
\hline M5 & II-59 & Ivans et al. (2001) & 4450 & 1.27 & 1.30 & $-1.15 \pm 0.04$ & $\ldots$ & 4443 & 1.32 & 1.83 & $-1.26 \pm 0.11$ & $+0.14 \pm 0.19$ \\
\hline M5 & G18447_0453 & Ramírez \& Cohen (2003) & 5275 & 3.15 & 1.44 & $-1.37 \pm 0.05$ & $+0.13 \pm 0.06$ & 5279 & 3.06 & 1.42 & $-1.23 \pm 0.11$ & $+0.17 \pm 0.12$ \\
\hline M5 & $1-\overline{1}$ & Ramírez \& Cohen (2003) & 4880 & 2.25 & 1.64 & $-1.30 \pm 0.03$ & $+0.24 \pm 0.08$ & 4903 & 2.14 & 1.63 & $-1.27 \pm 0.11$ & $+0.22 \pm 0.11$ \\
\hline M5 & IV-59 & Ivans et al. (2001) & 4229 & 0.79 & 2.10 & $-1.25 \pm 0.07$ & $\cdots$ & 4254 & 0.99 & 1.91 & $-1.34 \pm 0.11$ & $+0.14 \pm 0.12$ \\
\hline M5 & G18484_0316 & Ramírez \& Cohen (2003) & 4995 & 2.50 & 1.58 & $-1.38 \pm 0.03$ & $+0.22 \pm 0.06$ & 4980 & 2.41 & 1.57 & $-1.32 \pm 0.11$ & $+0.17 \pm 0.11$ \\
\hline
\end{tabular}

Notes. Some columns ([Si/Fe], $[\mathrm{Ca} / \mathrm{Fe}]$, and $[\mathrm{Ti} / \mathrm{Fe}]$ for both MRS and HRS) are suppressed in the printed edition.

(This table is available in its entirety in a machine-readable form in the online journal. A portion is shown here for guidance regarding its form and content.) 


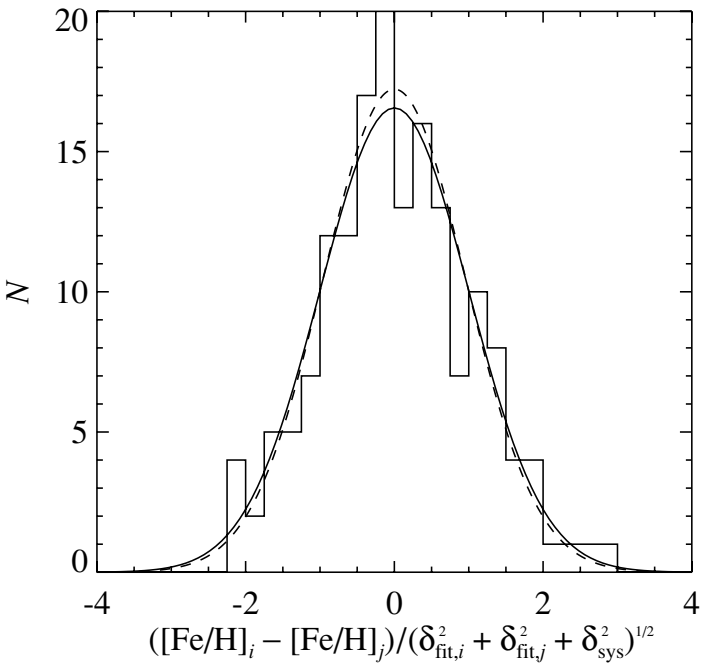

Figure 11. Distribution of differences between the repeat measurements of $[\mathrm{Fe} / \mathrm{H}]$ for 167 stars divided by the estimated error of the difference. The solid curve is a unit Gaussian with $\sigma=1$. The best-fit Gaussian (dashed line) has $\sigma=0.98$

Table 8

Adopted Solar Composition

\begin{tabular}{lc}
\hline \hline Element & $12+\log \epsilon$ \\
\hline $\mathrm{Mg}$ & 7.58 \\
$\mathrm{Si}$ & 7.55 \\
$\mathrm{Ca}$ & 6.36 \\
$\mathrm{Ti}$ & 4.99 \\
$\mathrm{Fe}$ & 7.52 \\
\hline
\end{tabular}

Notes. This composition is adopted from Anders \& Grevesse (1989), except for Fe. For justification of the adopted Fe solar abundance, see Sneden et al. (1992). The abundance of an element $\mathrm{X}$ is defined as its number density relative to hydrogen: $12+\log \epsilon_{\mathrm{X}}=$ $12+\log \left(n_{\mathrm{X}}\right)-\log \left(n_{\mathrm{H}}\right)$.

observed with DEIMOS. The GC and dSph stars were some of the targets on multi-slit masks, and the MW halo stars were observed through a long-slit mask. The one star in M92 was also observed through a long slit.

Different authors prefer different measurements of the solar compositions. We have placed all abundance measurements in this paper on the same scale by adjusting the published HRS values by the differences between the solar composition adopted by each HRS study and the solar composition given in Table 8 . The note at the bottom of the table explains our choice of solar composition.

All HRS studies share some techniques in common, but some aspects of the analyses differ. Table 9 summarizes the components of the HRS methods that change from study to study. The HRS studies we cite here all measure equivalent widths (EWs) of individual metal lines, and they compute abundances from those EWs, model atmospheres, and line lists. The line lists vary from study to study, causing typically small changes in derived abundances $(\lesssim 0.1 \mathrm{dex})$. Also, different studies use different codes to calculate model atmospheres, e.g., ATLAS9 (Kurucz 1993; Castelli \& Kurucz 2004) and MARCS (Gustafsson et al. 1975). The different codes used to calculate the abundances are given in footnote $\mathrm{b}$ of Table 9.

Most importantly, different HRS studies determine $T_{\text {eff }}$ and $\log g$ in different ways. Sometimes effective temperature is

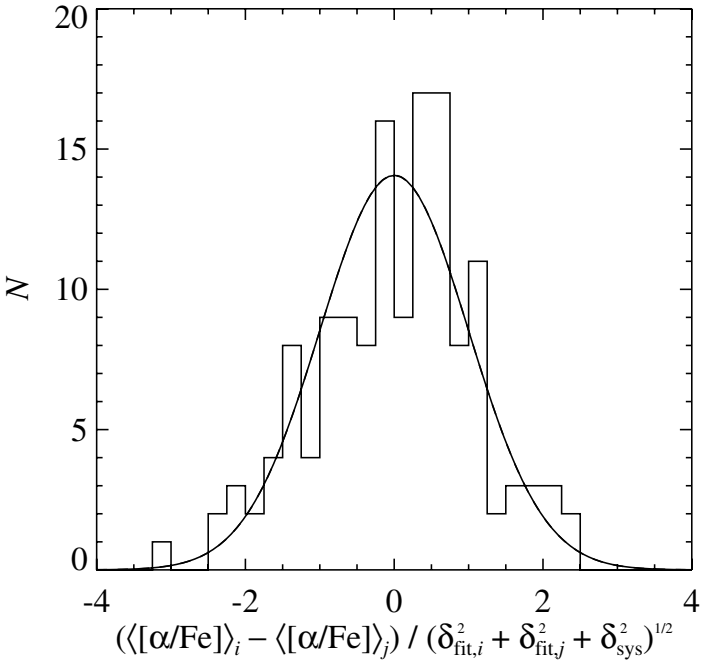

Figure 12. Distribution of differences between the repeat measurements of $\langle[\alpha / \mathrm{Fe}]\rangle$, which is the average of $[\mathrm{Mg} / \mathrm{Fe}],[\mathrm{Si} / \mathrm{Fe}],[\mathrm{Ca} / \mathrm{Fe}]$, and $[\mathrm{Ti} / \mathrm{Fe}]$, for 141 stars divided by the estimated error of the difference. The solid curve is a unit Gaussian with $\sigma=1$. The best-fit Gaussian (dashed line, exactly overlying the solid line) has $\sigma=1.00$

determined through excitation equilibrium, wherein $T_{\text {eff }}$ is adjusted to minimize the trend of abundance with the excitation potential of the Fe transition. Surface gravity is sometimes determined through ionization balance, wherein $\log g$ is adjusted until the abundance measured from lines of $\mathrm{Fe}$ I agrees with the abundance measured from lines of Fe II. Gray (2008) explains these methods in detail. Some authors of HRS studies use photometric determinations of $T_{\text {eff }}$ and $\log g$ only, relying on empirical calibrations or theoretical isochrones. For a discussion on the merits and disadvantages of photometrically determined atmospheric parameters, we refer the reader to Ivans et al. (2001).

It is tempting to assume that our HRS comparison set is a flawless standard, but the heterogeneity of the sources of these abundance estimates introduces systematic error. Measurement uncertainties from HRS are generally smaller than from MRS, but systematic offsets between studies arise from different choices of methods of determining atmospheric parameters, line lists, model atmospheres, and spectral synthesis codes. Table 9 is meant to illustrate the diversity of ways to measure elemental abundances spectroscopically. These differences should be borne in mind when examining the HRS measurements presented below. The sample sizes are often too small to make a meaningful statistical quantification of bias between HRS studies of the same stars or stellar systems. Careful attention should be paid to potential systematic offsets between HRS studies. Although our analysis is also subject to its own random uncertainties and systematic errors, one of the principal advantages of our sample is that the abundances of all of the stars have been measured in a homogeneous fashion, eliminating most relative systematic offsets from star to star.

Table 10 lists the differences and standard deviations between MRS and HRS measurements for all three types of stellar systems. The quantities are very similar to comparisons between different HRS measurements of the same stars (e.g., Cohen et al. 2008, their Appendix B).

Figures 13-18 show the comparison between HRS measurements ( $x$-axes) and MRS measurements ( $y$-axes). The points are coded by their membership in GCs, the MW halo field, or dSphs. Coding the points by individual system — such as the identity of 
Table 9

Previously Published HRS Abundance Methods

\begin{tabular}{|c|c|c|c|c|c|}
\hline Reference & Atmospheres $^{\mathrm{a}}$ & Code $^{b}$ & $T_{\text {eff }}{ }^{\mathrm{c}}$ & $\log g^{\mathrm{d}}$ & $\xi^{\mathrm{e}}$ \\
\hline \multicolumn{6}{|c|}{ Globular clusters } \\
\hline Cohen \& Meléndez (2005a, 2005b) & ATLAS9 & MOOG & phot & phot & $\operatorname{spec}^{f}$ \\
\hline Gratton \& Ortolani (1989) & Bell et al. (1976) & WIDTH2 & phot & phot & spec \\
\hline Ivans et al. (2001) & MARCS & MOOG & $\operatorname{spec}^{g}$ & spec $^{g}$ & spec \\
\hline Mishenina et al. (2003) & ATLAS9 & WIDTH9 & spec & spec & spec \\
\hline Ramírez \& Cohen $(2002,2003)$ & ATLAS9 & MOOG & phot & phot & $\operatorname{spec}^{h}$ \\
\hline Sneden et al. $(1997,2000)$ & MARCS & MOOG & spec & spec & spec \\
\hline Sneden et al. (2004) & MARCS & MOOG & spec & phot & spec \\
\hline \multicolumn{6}{|c|}{ Halo Field Stars } \\
\hline Carretta et al. (2002) & ATLAS9 & unknown ${ }^{\mathrm{i}}$ & phot & phot & spec \\
\hline Cohen et al. $(2006,2008)$ & ATLAS9 & MOOG & phot & phot & phot $^{\mathrm{j}}$ \\
\hline Fulbright (2000) & ATLAS9 & MOOG & spec & spec & spec \\
\hline Johnson (2002) & ATLAS9 & MOOG & spec & spec & spec \\
\hline Lai et al. $(2004,2007)$ & ATLAS9 & MOOG & phot & phot & evolutionary \\
\hline$\underline{\text { Pilachowski et al. (1996) }}$ & MARCS & MOOG & phot & phot & spec \\
\hline \multicolumn{6}{|c|}{ dSphs } \\
\hline Cohen \& Huang $(2009,2010)$ & ATLAS9 & MOOG & spec & spec & spec \\
\hline Frebel et al. (2010a, 2010b) & ATLAS9 & MOOG & spec & spec & spec \\
\hline Fulbright et al. (2004) & ATLAS9 & MOOG & phot & phot & spec \\
\hline Geisler et al. (2005) & MARCS & MOOG & phot & spec & spec \\
\hline Koch et al. (2008) & ATLAS9 & MOOG & spec & spec & spec \\
\hline Letarte et al. (2010) & MARCS & CALRAI & phot & phot & spec \\
\hline Sadakane et al. (2004) & ATLAS9 & SPTOOL & spec & spec & spec \\
\hline Shetrone et al. (2001, 2003, 2009) & MARCS & MOOG & spec & spec & spec \\
\hline Simon et al. (2010) & ATLAS9 & MOOG & phot $^{\mathrm{k}}$ & phot $^{\mathrm{k}}$ & spec \\
\hline
\end{tabular}

Notes.

${ }^{a}$ ATLAS9: Kurucz (1993) or Castelli \& Kurucz (2004), also http://kurucz.harvard.edu/grids.html; MARCS: Gustafsson et al. (1975, 2003, 2008).

b MOOG: Sneden (1973); WIDTH2: Gratton (1982); WIDTH9: Kurucz (2005); CALRAI: Spite (1967); SPTOOL: Y. Takeda (unpublished, but based on WIDTH9).

${ }^{c}$ phot: empirical color- $T_{\text {eff }}$ relation or model isochrones; spec: Fe I excitation equilibrium.

${ }^{\mathrm{d}}$ phot: determined from model isochrones or $T_{\text {eff }}$, with luminosity based on bolometric corrections, and assumption of the stellar mass; spec: Fe I and Fe II ionization balance.

${ }^{\mathrm{e}}$ spec: based on removing abundance trends with equivalent width; evolutionary: $\xi$ assigned based on position in the color-magnitude diagram

${ }^{\mathrm{f}}$ Because $\xi$ for all stars was similar, Cohen \& Meléndez (2005b) assumed $2.0 \mathrm{~km} \mathrm{~s}^{-1}$ for all stars in NGC 7492.

$\mathrm{g}$ Photometric values were also derived, but we have adopted the spectroscopic values and corresponding abundances.

${ }^{\mathrm{h}} \mathrm{A} T_{\mathrm{eff}}-\xi$ relation derived from the brighter stars was assumed for the fainter stars.

${ }^{\mathrm{i}}$ Although their paper does not mention it, these authors typically use MOOG.

${ }^{\mathrm{j}}$ The value of $\xi$ was set to $1.6-1.8 \mathrm{~km} \mathrm{~s}^{-1}$ with variation depending on $T_{\text {eff }}$.

${ }^{\mathrm{k}}$ Spectroscopic values were also derived, but the authors preferred the photometric values.

Table 10

Differences Between MRS and HRS Atmospheric Parameters and Abundances

\begin{tabular}{lcccc}
\hline \hline \multicolumn{1}{c}{ Quantity } & GCs & MW Halo & dSphs & All \\
\hline$\left\langle\delta T_{\text {eff }}\right\rangle(\mathrm{K})$ & $-29(79)$ & $+11(129)$ & $-40(132)$ & $-27(115)$ \\
$\langle\delta \log g\rangle\left(\mathrm{cm} \mathrm{s}^{-2}\right)$ & $+0.06(0.18)$ & $\mathrm{a}$ & $+0.05(0.41)$ & $+0.05(0.33)$ \\
$\langle\delta \xi\rangle\left(\mathrm{km} \mathrm{s}^{-1}\right)$ & $+0.1(0.3)$ & $-0.2(0.2)$ & $-0.2(0.3)$ & $-0.1(0.3)$ \\
$\langle\delta[\mathrm{Fe} / \mathrm{H}]\rangle$ & $-0.09(0.13)$ & $-0.14(0.17)$ & $-0.04(0.15)$ & $-0.07(0.15)$ \\
$\langle\delta[\mathrm{Mg} / \mathrm{Fe}]\rangle$ & $-0.07(0.24)$ & $+0.05(0.24)$ & $-0.06(0.18)$ & $-0.05(0.20)$ \\
$\langle\delta[\mathrm{Si} / \mathrm{Fe}]\rangle$ & $+0.04(0.17)$ & $+0.19(0.18)$ & $-0.16(0.28)$ & $-0.04(0.26)$ \\
$\langle\delta[\mathrm{Ca} / \mathrm{Fe}]\rangle$ & $+0.02(0.16)$ & $-0.04(0.17)$ & $-0.03(0.23)$ & $-0.01(0.20)$ \\
$\langle\delta[\mathrm{Ti} / \mathrm{Fe}]\rangle$ & $-0.04(0.14)$ & $+0.02(0.25)$ & $-0.05(0.24)$ & $-0.04(0.22)$ \\
$\langle\delta\langle[\alpha / \mathrm{Fe}]\rangle\rangle^{\mathrm{b}}$ & $+0.01(0.14)$ & $+0.02(0.17)$ & $-0.05(0.19)$ & $-0.02(0.16)$ \\
\hline
\end{tabular}

Notes. Positive numbers indicate that the MRS measurements are larger than the HRS measurements. Numbers in parentheses are standard deviations.

${ }^{a}$ For MW halo stars, for which distances are poorly known, $(\log g)_{\text {MRS }}$ was set equal to $(\log g)_{\text {HRS }}$.

b $\langle[\alpha / \mathrm{Fe}]\rangle$ is an average of the measurements of $[\mathrm{Mg} / \mathrm{Fe}],[\mathrm{Si} / \mathrm{Fe}],[\mathrm{Ca} / \mathrm{Fe}]$, and $[\mathrm{Ti} / \mathrm{Fe}]$, but only for $\langle[\alpha / \mathrm{Fe}]\rangle$

measurements with estimated uncertainties of less than 0.5 dex. 

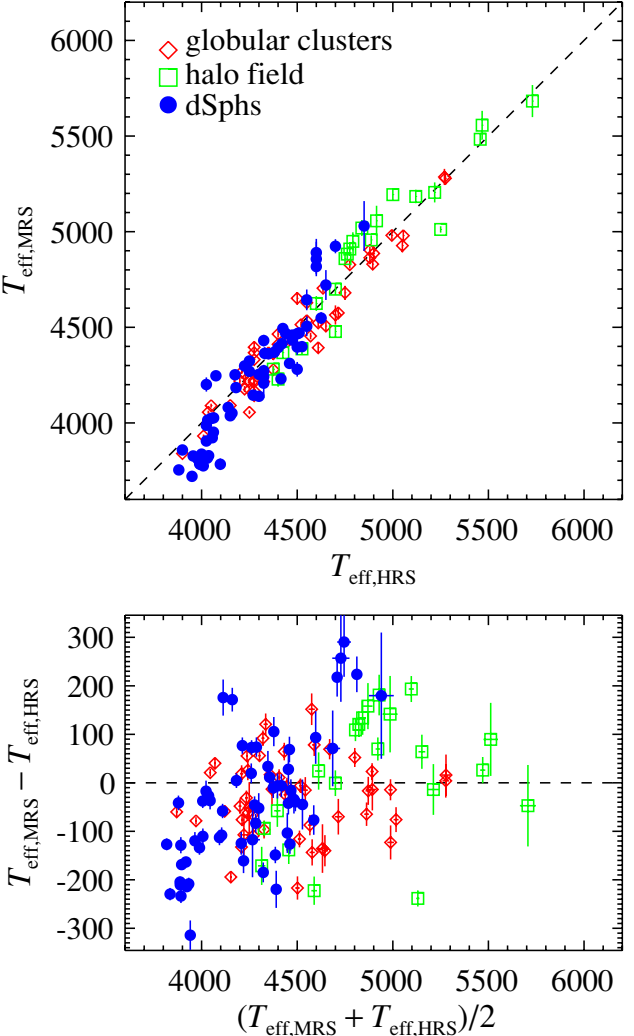

Figure 13. Top: comparison between effective temperature $\left(T_{\mathrm{eff}}\right)$ used in previous HRS abundance analyses and $T_{\text {eff }}$ used for this work's MRS abundance analysis. The dashed line is one-to-one. The shape and color of the plotting symbol indicates the type of stellar system of which the star is a member. The error bars represent the error on the MRS fit to $T_{\text {eff }}$, and they are not intended to represent all sources of error. Bottom: residuals between $T_{\text {eff,MRS }}$ and $T_{\text {eff,HRS }}$ vs. the average of $T_{\text {eff,MRS }}$ and $T_{\text {eff,HRS }}$

(A color version of this figure is available in the online journal.)

the $\mathrm{GC}$ or $\mathrm{dSph}$ - and also by HRS reference is also instructive, but the plots contain too many points for this coding to be feasible. Tables 7 and 9 contain more complete descriptions of the comparisons between MRS and HRS measurements.

The bottom panels of Figures 13-18 show the differences between MRS and HRS measurements $(y-x)$. Instead of plotting $y-x$ versus $x$, we have plotted $y-x$ versus $(y+x) / 2$, the average of $x$ and $y$. This is effectively a scaled orthogonal distance from the one-to-one line. (A pure rotation of the upper panel, $y$ versus $x$, would be $(y-x) / \sqrt{2}$ versus $(y+x) / \sqrt{2}$, but $y-x$ is easier to interpret than $(y-x) / \sqrt{2}$.) We have chosen the orthogonal distance as the abscissa because a plot of $y-x$ versus $x$ would show trends even if $x$ and $y$ are drawn from the same distribution with random scatter. In fact, any random uncertainty in $x$ would cause a downward sloping trend in $y-x$ versus $x$. In the orthogonal residual plots, random scatter along the oneto-one line does not produce a trend as long as the magnitude of the scatter in $x$ is close to magnitude of the scatter in $y$. The uncertainties in the MRS measurements are generally slightly larger than the uncertainties in the HRS measurements, but not enough to produce these trends in the orthogonal residuals.

Figure 13 compares $T_{\text {eff,HRS }}$ and $T_{\text {eff,MRS }}$. The average difference in $T_{\text {eff }}$ between MRS and HRS depends on the source of the HRS measurement. As one example, consider the MW halo field star sample. Johnson (2002) calculate $T_{\text {eff }}$ spectroscopically, whereas Lai et al. $(2004,2007)$ calculate $T_{\text {eff }}$ photometrically. Our measurements of $T_{\text {eff }}$ are typically $\sim 100 \mathrm{~K}$ below
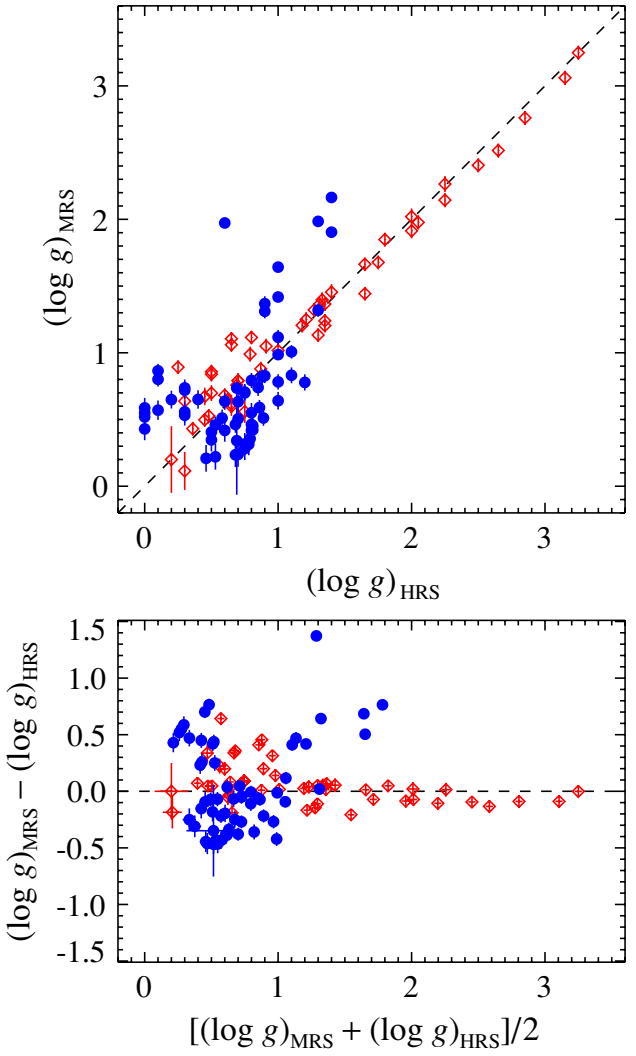

Figure 14. Same as Figure 13 except for surface gravity $(\log g)$ instead of $T_{\text {eff }}$. The error bars represent photometric uncertainties and isochrone modeling errors.

(A color version of this figure is available in the online journal.)

those of Johnson (2002) and $\sim 100 \mathrm{~K}$ above those of Lai et al. $(2004,2007)$. The differences possibly result from the different methods of measuring $T_{\text {eff }}$.

As another example of a trend seen with the source of the HRS measurements, consider the study of Frebel et al. (2010b). Five of the six stars in the Ursa Major II and Coma Berenices data set have $T_{\text {eff,MRS }}$ at least $175 \mathrm{~K}$ higher than $T_{\text {eff,HRS }}$. Furthermore, $\xi_{\text {MRS }}$ is between 0.3 and $0.8 \mathrm{~km} \mathrm{~s}^{-1}$ less than $\xi_{\text {HRS }}$ for all of the stars in Frebel et al.'s sample. Our measurements of $[\mathrm{Fe} / \mathrm{H}]_{\mathrm{MRS}}$ for the five stars with the significantly different temperature measurements exceed $[\mathrm{Fe} / \mathrm{H}]_{\mathrm{HRS}}$ by $0.1-0.4$ dex. In contrast to our measurements, Frebel et al. used a blue spectral range, higher resolution, and spectroscopically derived surface gravities and microturbulent velocities. Our choices are not better, simply different. The differences partly explain our different offsets from different studies.

The standard deviation of $[\mathrm{Fe} / \mathrm{H}]_{\mathrm{MRS}}-[\mathrm{Fe} / \mathrm{H}]_{\mathrm{HRS}}$ is 0.15 . The minimum uncertainty that we quote on $[\mathrm{Fe} / \mathrm{H}]_{\mathrm{MRS}}$ (in the limit of infinite spectral $\mathrm{S} / \mathrm{N}$ ) is 0.113 . The small difference between our minimum estimate of uncertainty and the typical difference between MRS and HRS measurements-which includes the error on $[\mathrm{Fe} / \mathrm{H}]_{\mathrm{HRS}}$ in addition to the error on $[\mathrm{Fe} / \mathrm{H}]_{\mathrm{MRS}}$-indicates that we have not underestimated our measurement uncertainties, even on an absolute scale. Furthermore, Figure 16 shows no systematic trend in $[\mathrm{Fe} / \mathrm{H}]_{\mathrm{MRS}}-$ $[\mathrm{Fe} / \mathrm{H}]_{\mathrm{HRS}}$ as a function of $[\mathrm{Fe} / \mathrm{H}]$. Therefore, our MRS measurements of $[\mathrm{Fe} / \mathrm{H}]$ are consistent with $\mathrm{HRS}$ measurements at least over the range $-4.0<[\mathrm{Fe} / \mathrm{H}]<-0.5$.

The MRS $\alpha$ element abundances also agree with HRS measurements. (However, we added a constant to $[\mathrm{Si} / \mathrm{Fe}]_{\mathrm{MRS}}$ 

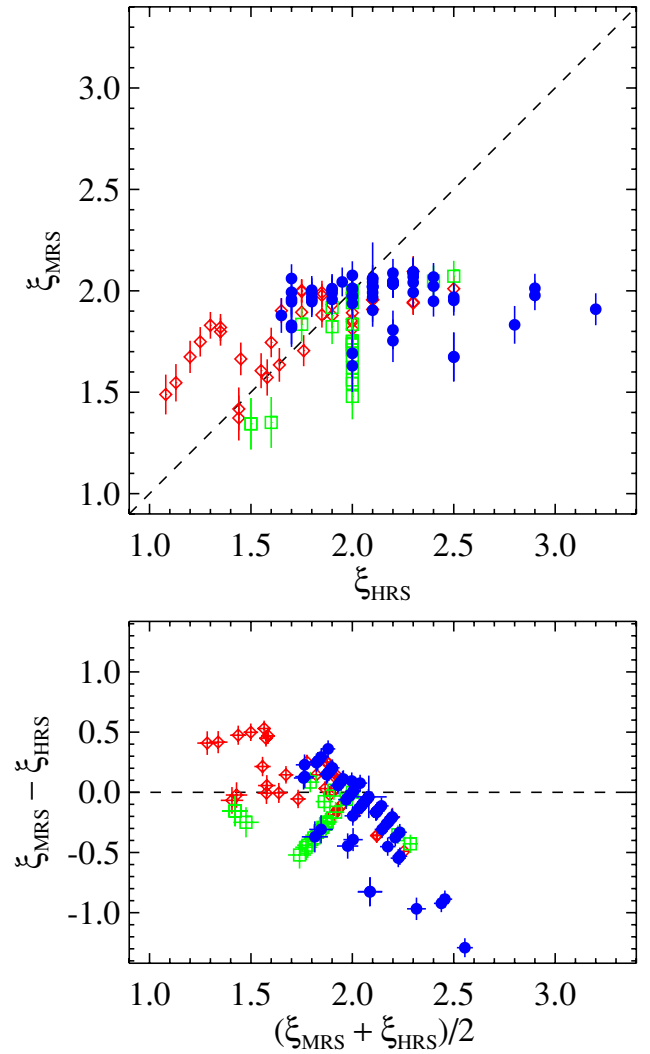

Figure 15. Same as Figure 13 except for microturbulent velocity $(\xi)$ instead of $T_{\text {eff }}$. The error bars are found by propagating the error on $\log g$ through the equation to determine $\xi$ from $\log g$ (Equation (2) of Paper I).

(A color version of this figure is available in the online journal.)

to force better agreement with $[\mathrm{Si} / \mathrm{Fe}]_{\mathrm{HRS}}$. See Section 3.3.) The standard deviation of the differences between MRS and HRS $\langle[\alpha / \mathrm{Fe}]\rangle$ (an average of the four measured $\langle[\alpha / \mathrm{Fe}]\rangle$ ratios) is 0.16 , about the same as the standard deviation for $[\mathrm{Fe} / \mathrm{H}]$. The number of detectable $\mathrm{Mg}, \mathrm{Si}, \mathrm{Ca}$, and Ti absorption lines in the DEIMOS spectral range is comparable to the number of $\mathrm{Fe}$ absorption lines for red giants. Therefore, it is reassuring that the precision of the $\langle[\alpha / \mathrm{Fe}]\rangle_{\text {MRS }}$ measurements is comparable to the precision of the $[\mathrm{Fe} / \mathrm{H}]_{\mathrm{MRS}}$ measurements.

We point out in particular an interesting feature of the individual alpha element ratios concerning GC stars (red points in Figure 17). Internal variations in $\mathrm{Ca}$ and $\mathrm{Ti}$ have not been detected in the GCs presented here. Furthermore, the $[\mathrm{Ca} / \mathrm{Fe}]$ and $[\mathrm{Ti} / \mathrm{Fe}]$ ratios vary little from cluster to cluster. Therefore, it is expected that we see small or zero correlation between MRS and HRS measurements for these elements. In fact, the linear Pearson correlation coefficients between MRS and HRS measurements are 0.33 and 0.26 for $[\mathrm{Ca} / \mathrm{Fe}]$ and $[\mathrm{Ti} / \mathrm{Fe}]$, respectively. On the other hand, $[\mathrm{Mg} / \mathrm{Fe}]$ shows a significant spread within many GCs (e.g., Gratton et al. 2004). As a result, we see a correlation between $[\mathrm{Mg} / \mathrm{Fe}]_{\mathrm{MRS}}$ and $[\mathrm{Mg} / \mathrm{Fe}]_{\mathrm{HRS}}$. The correlation coefficient is 0.41 . In other words, the $\alpha$ element ratio measurements from DEIMOS can sort out which of the four $[\mathrm{X} / \mathrm{Fe}]$ ratios has a dispersion between GCs.

The large sample of stars observed with at least two independent measurements provides a unique opportunity to examine the influence of errors in atmospheric parameters on $[\mathrm{Fe} / \mathrm{H}]$. Figures 19-21 show how differences in $T_{\text {eff }}, \log g$, and $\xi$ affect the measurement of $[\mathrm{Fe} / \mathrm{H}]$. Each figure shows the least-squares linear fit. The vertical error bars are the quadrature sum of the
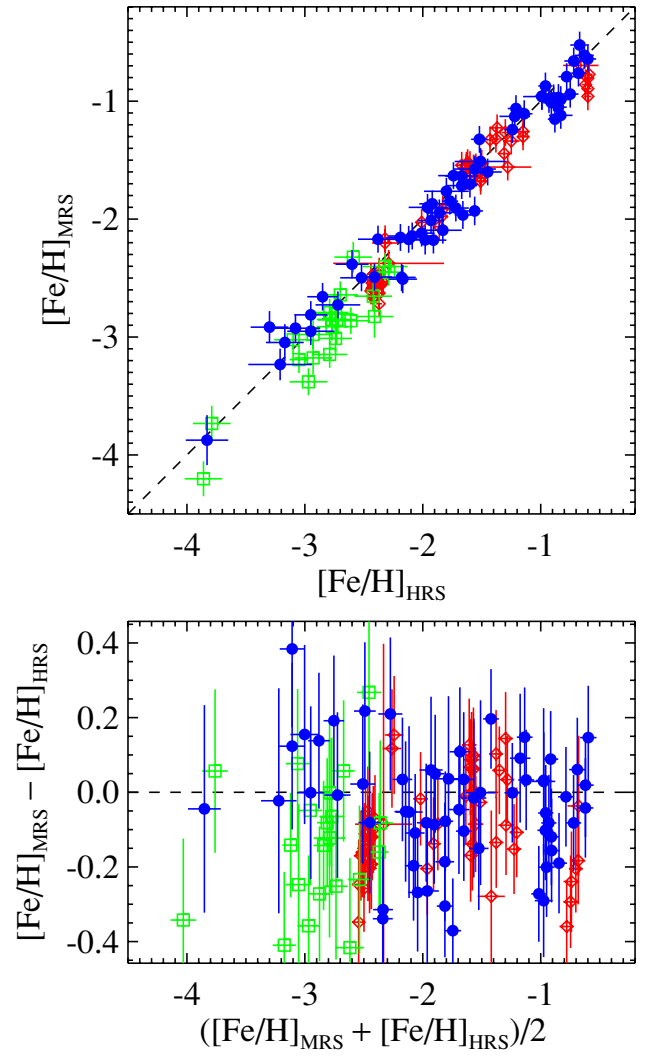

Figure 16. Comparison between $[\mathrm{Fe} / \mathrm{H}]$ derived from previous HRS abundance analyses and $[\mathrm{Fe} / \mathrm{H}]$ derived from this work's MRS abundance analysis. Colors are the same as in Figure 13.

(A color version of this figure is available in the online journal.)

total errors on $[\mathrm{Fe} / \mathrm{H}]$ from MRS and HRS, including the error introduced by uncertainty in atmospheric parameters. Horizontal error bars are not shown because most HRS studies do not include errors on atmospheric parameters. Not surprisingly, $T_{\text {eff }}$ is the atmospheric parameter that most affects the measurement of $[\mathrm{Fe} / \mathrm{H}]$. In general, underestimating $T_{\text {eff }}$ leads to an underestimate of $[\mathrm{Fe} / \mathrm{H}]$. Reassuringly, the intercept of the least-squares linear fit to $\left([\mathrm{Fe} / \mathrm{H}]_{\mathrm{MRS}}-[\mathrm{Fe} / \mathrm{H}]_{\mathrm{HRS}}\right)$ versus $\left(T_{\text {eff,MRS }}-T_{\text {eff,HRS }}\right)$ is close to zero $(-0.06 \pm 0.01)$. In other words, if $T_{\text {eff }}$ were determined perfectly, $[\mathrm{Fe} / \mathrm{H}]_{\mathrm{MRS}}$ and $[\mathrm{Fe} / \mathrm{H}]_{\mathrm{HRS}}$ would agree extremely well. Surface gravity does not have a strong influence on the measurement of $[\mathrm{Fe} / \mathrm{H}]$, and microturbulent velocity has a moderately strong influence. Underestimating $\xi$ leads to an overestimate of $[\mathrm{Fe} / \mathrm{H}]$.

In order to quantify the total effect of errors on atmospheric parameters, we have identified the linear combination of $T_{\text {eff }}$, $\log g$, and $\xi$ differences that minimizes the scatter about the least-squares linear fit to $\left([\mathrm{Fe} / \mathrm{H}]_{\mathrm{MRS}}-[\mathrm{Fe} / \mathrm{H}]_{\mathrm{HRS}}\right)$. Figure 22 shows the result. To remove dimensionality, $\delta T_{\text {eff }}, \delta(\log g)$, and $\delta \xi$ have been normalized by their standard deviations. As expected, $T_{\text {eff }}$ has the most influence on the $[\mathrm{Fe} / \mathrm{H}]$ measurement by far. Surface gravity and $\xi$ have about one half of the influence of $T_{\text {eff }}$. The rms scatter about the line is $0.13 \mathrm{dex}$, compared to $0.15 \mathrm{dex}$, which is the rms scatter in $\left([\mathrm{Fe} / \mathrm{H}]_{\mathrm{MRS}}-[\mathrm{Fe} / \mathrm{H}]_{\mathrm{HRS}}\right)$ without removing the effect of errors from atmospheric parameters. Therefore, uncertainty in $T_{\text {eff }}$ and $\xi$ do inflate the error on $[\mathrm{Fe} / \mathrm{H}]$. This result possibly indicates that $\mathrm{S} / \mathrm{N}$ (for these bright comparison stars) and spectral resolution are not limiting the precision of $[\mathrm{Fe} / \mathrm{H}]_{\mathrm{MRS}}$. Instead, improving the determinations of $T_{\text {eff }}$ and $\xi$ would do much to improve the measurement of 

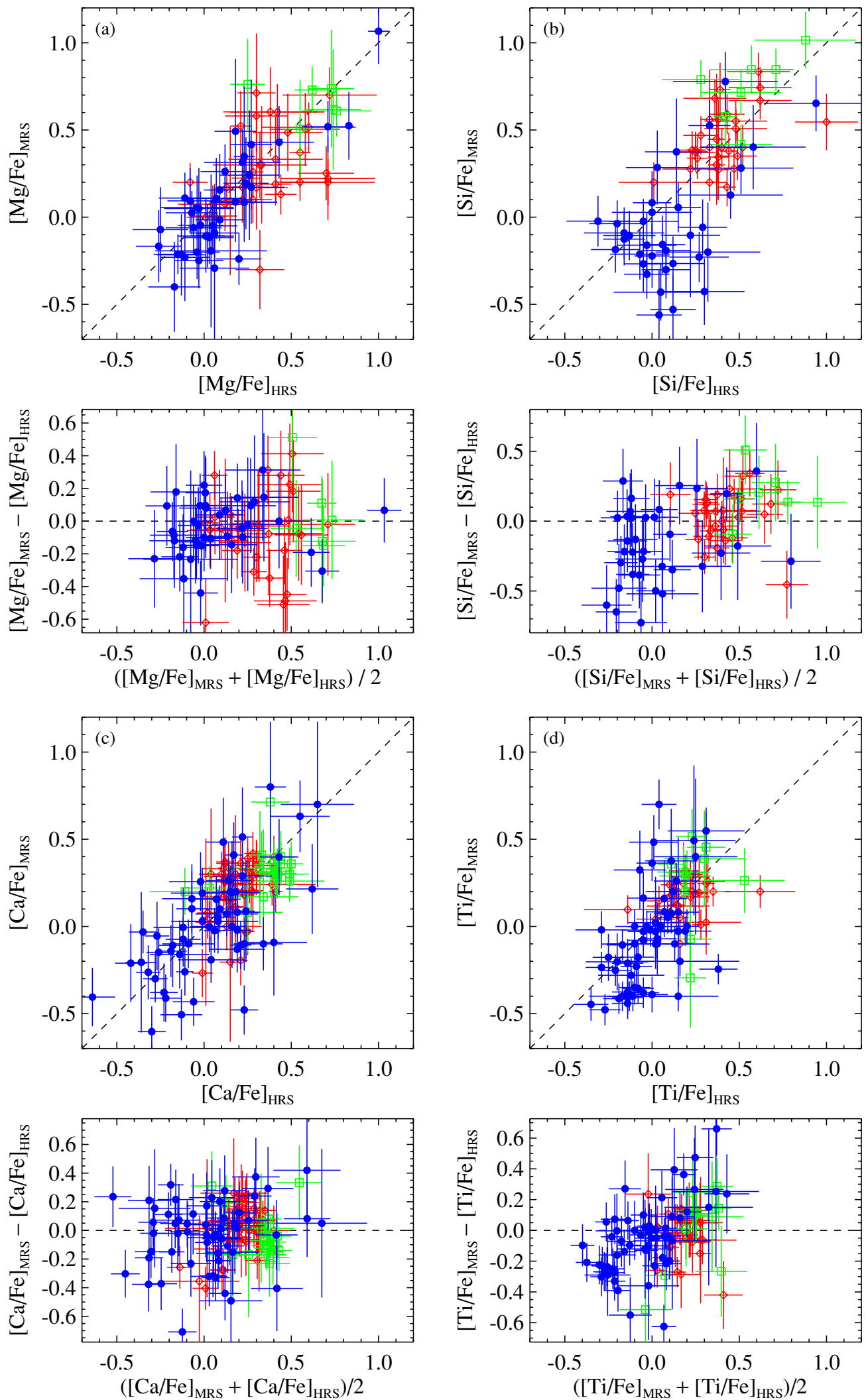

Figure 17. Same as Figure 16 except for (a) $[\mathrm{Mg} / \mathrm{Fe}]$, (b) $[\mathrm{Si} / \mathrm{Fe}]$, (c) $[\mathrm{Ca} / \mathrm{Fe}]$, and (d) $[\mathrm{Ti} / \mathrm{Fe}]$. (A color version of this figure is available in the online journal.)

$[\mathrm{Fe} / \mathrm{H}]_{\mathrm{MRS}}$. The problem of measuring $T_{\mathrm{eff}}$ and $\xi$ is not unique to MRS. Table 9 shows that many HRS studies, particularly for the more distant stars, employ photometry-at least in part—for determining $T_{\text {eff }}, \log g$, and $\xi$, as we do.

\section{SUMMARY}

We have presented a catalog of $\mathrm{Fe}, \mathrm{Mg}, \mathrm{Si}, \mathrm{Ca}$, and $\mathrm{Ti}$ abundance measurements for 2961 stars in eight dwarf satellite 

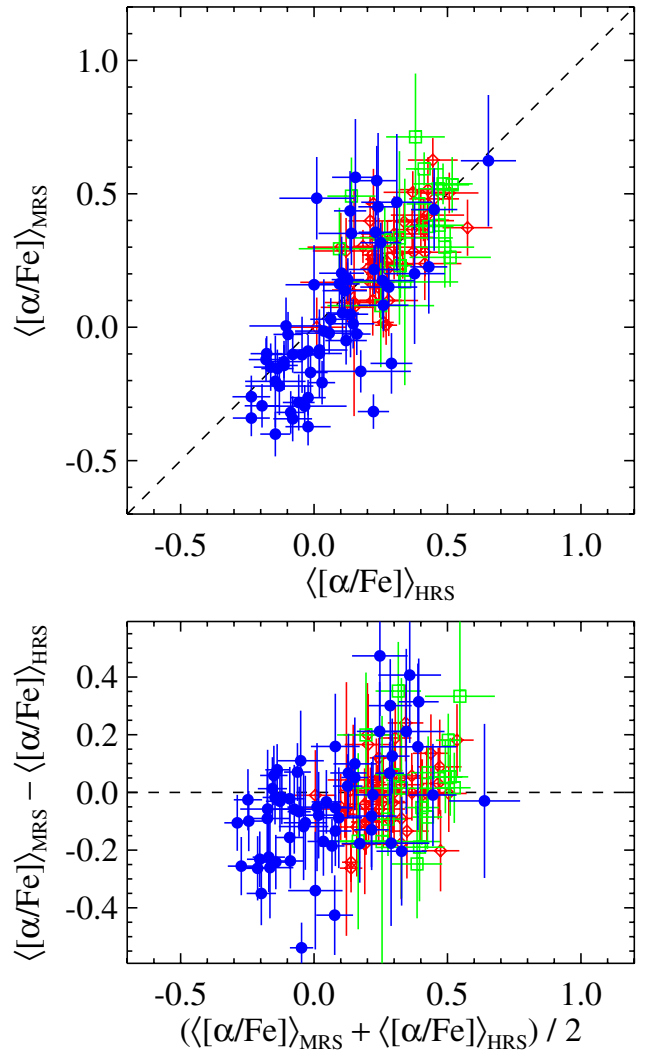

Figure 18. Same as Figure 17 except for an average of the $\alpha$ elements. The average includes only those $\langle[\alpha / \mathrm{Fe}]\rangle$ measurements with estimated uncertainties less than $0.5 \mathrm{dex}$.

(A color version of this figure is available in the online journal.)

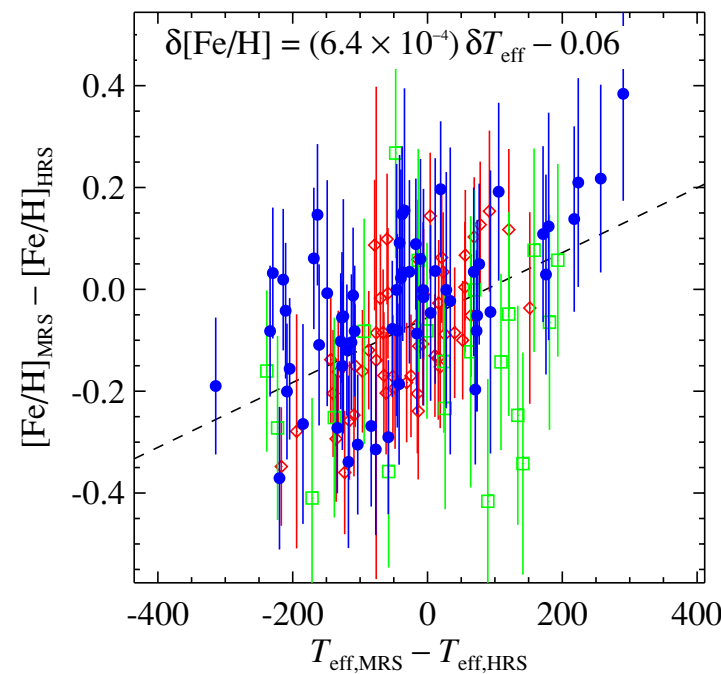

Figure 19. Covariance between errors in $T_{\text {eff }}$ and $[\mathrm{Fe} / \mathrm{H}]$. The $x$-axis shows the difference between the MRS and HRS measurements of $T_{\text {eff }}$, and the $y$-axis shows the same for $[\mathrm{Fe} / \mathrm{H}]$. The colors and shapes of the points are the same as in Figure 16. The dashed line is a least-squares fit. The strong covariance between these differences illustrates the degeneracy between $T_{\text {eff }}$ and $[\mathrm{Fe} / \mathrm{H}]$ inherent in stellar spectral analysis.

(A color version of this figure is available in the online journal.)

galaxies of the MW. MRS from the Keck/DEIMOS spectrograph provided the throughput and multiplexing necessary to perform these measurements for the large number of faint, distant stars. The majority of these stars are inaccessible to highresolution spectrographs even on the largest telescopes. The measurements relied on a spectral synthesis technique discussed

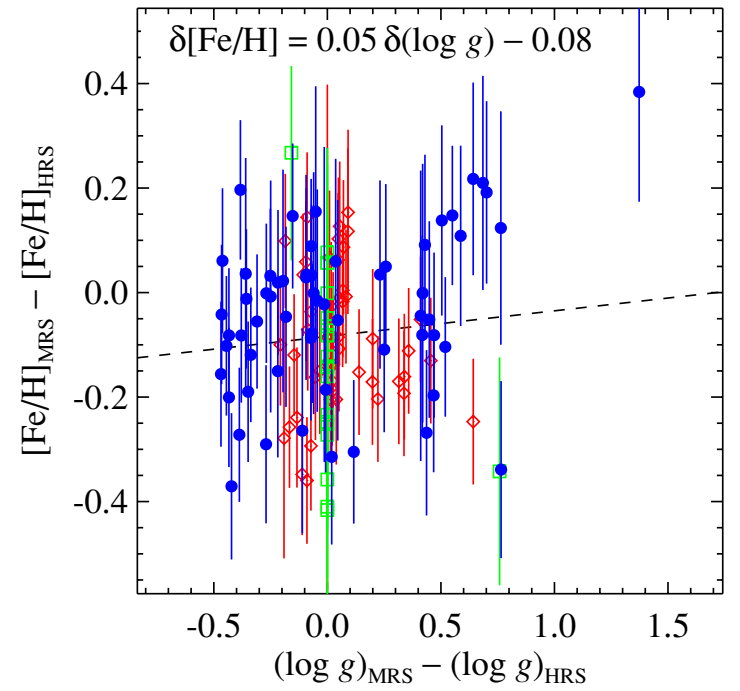

Figure 20. Covariance between errors in $\log g$ and $[\mathrm{Fe} / \mathrm{H}]$. See Figure 19 for further explanation. The weak covariance between these differences illustrates the slight degeneracy between $\log g$ and $[\mathrm{Fe} / \mathrm{H}]$ inherent in stellar atmosphere analysis of mostly neutral metal lines. The combination of very discrepant $T_{\text {eff }}$, $\log g$, and $\xi$ causes the outlier with $[\mathrm{Fe} / \mathrm{H}]_{\mathrm{MRS}}-[\mathrm{Fe} / \mathrm{H}]_{\mathrm{MRS}}=+0.4$.

(A color version of this figure is available in the online journal.)

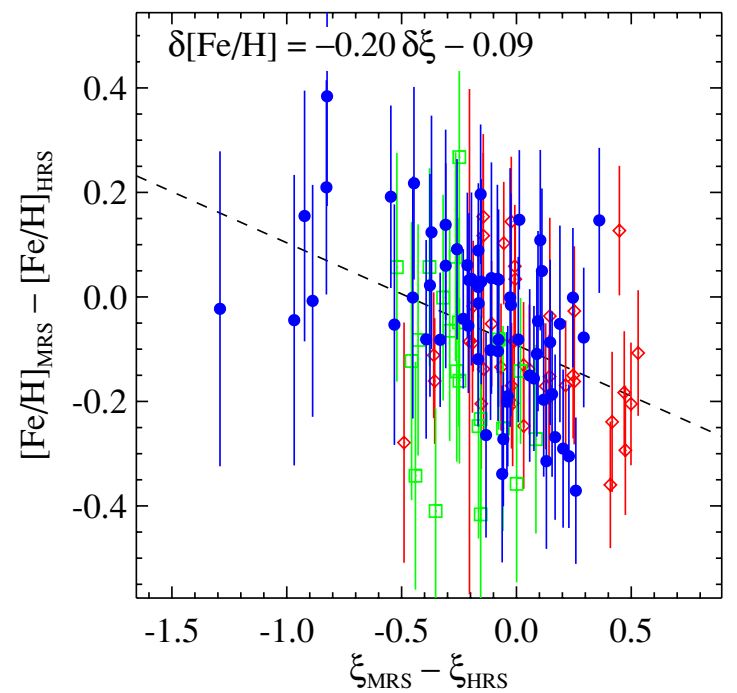

Figure 21. Covariance between errors in $\xi$ and $[\mathrm{Fe} / \mathrm{H}]$. See Figure 19 for further explanation. The strong anticorrelation illustrates the degeneracy between $\xi$ and $[\mathrm{Fe} / \mathrm{H}]$ inherent in stellar atmosphere analysis.

(A color version of this figure is available in the online journal.)

previously (KGS08 and Paper I) with some modifications detailed in this paper.

We have estimated the uncertainty on every measurement of $T_{\text {eff }}, \log g,[\mathrm{Fe} / \mathrm{H}],[\mathrm{Mg} / \mathrm{Fe}],[\mathrm{Si} / \mathrm{Fe}],[\mathrm{Ca} / \mathrm{Fe}]$, and $[\mathrm{Ti} / \mathrm{Fe}]$. The uncertainties on the abundances include the effect of spectral noise, spectral modeling uncertainties, and uncertainties in atmospheric parameters (effective temperature and surface gravity). The estimated uncertainties were shown to be accurate based on duplicate observations of stars in the scientific targets, dwarf galaxies.

Finally, we have quantified the accuracy of our mediumresolution measurements by observing with DEIMOS a sample of stars with high-resolution spectroscopic measurements. We deliberately targeted stars in dwarf galaxies with previous HRS measurements, and we observed stars in GCs and in the field of the MW stellar halo. The mean difference in $[\mathrm{Fe} / \mathrm{H}]$ and 


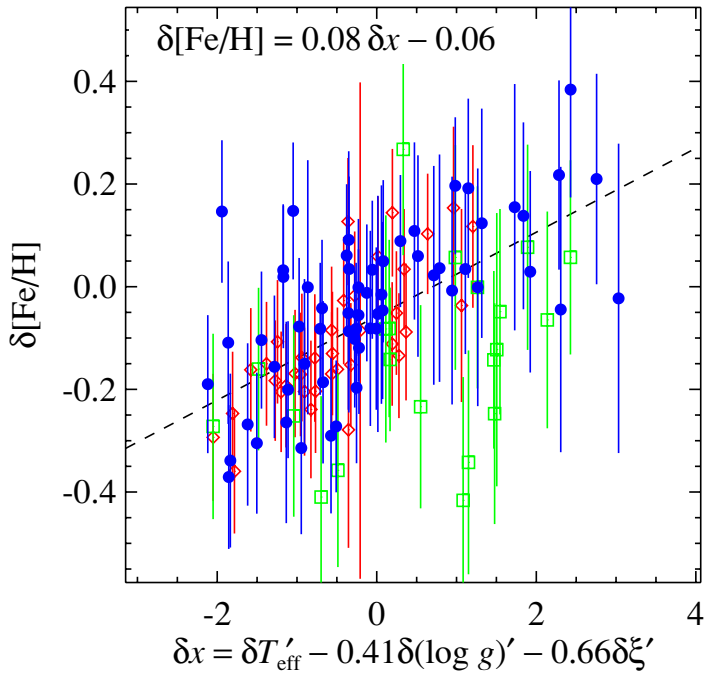

Figure 22. Covariance between $[\mathrm{Fe} / \mathrm{H}]$ and the linear combination of atmospheric parameters that minimizes the $\chi^{2}$ of the linear fit to differences between the MRS and HRS measurements of $[\mathrm{Fe} / \mathrm{H}]$. The symbol $\delta$ represents the difference between MRS and HRS measurements, as in Figures 19-21. The primes in the $x$-axis label indicate that the quantities have been normalized by their standard deviations $\left(115 \mathrm{~K}, 0.33\right.$, and $0.3 \mathrm{~km} \mathrm{~s}^{-1}$ for $\delta T_{\text {eff }}, \delta(\log g)$, and $\delta \xi$, respectively).

(A color version of this figure is available in the online journal.)

$\langle[\alpha / \mathrm{Fe}]\rangle$ between the HRS and MRS measurements of these 132 stars is -0.07 and -0.02 , with standard deviations of 0.15 and 0.16 .

The next paper in this series focuses on the metallicity evolution of the individual dSphs. We fit chemical evolution models to the metallicity distributions. The shapes of the distributions and parameters of the fits show trends with dSph luminosity. The following paper in the series addresses the star formation timescale and chemical evolution as revealed by the distribution of the $\alpha$ elements.

The authors thank the referee, Piercarlo Bonifacio, for his thoughtful suggestions, which improved this manuscript. We gratefully acknowledge Sandra Faber, Ricardo Schiavon, and Michael Cooper of the DEEP2 team for acquiring Keck/ DEIMOS spectroscopy of bright Milky Way halo field stars during nights of poor transparency. We also thank Peter Stetson for providing additional globular cluster photometry and Bob Kraft for helpful discussions and for providing some of the globular cluster spectroscopy for this work. The generation of synthetic spectra made use of the University of California Santa Cruz Pleiades supercomputer and the Yale High Performance Computing cluster Bulldog. We thank Joel Primack for sharing his allocation of Pleiades supercomputer time and Mario Jurić for a helpful discussion on the mass of the components of the Milky Way.

Support for this work was provided by NASA through Hubble Fellowship grant HST-HF-01233.01 awarded to E.N.K. by the Space Telescope Science Institute, which is operated by the Association of Universities for Research in Astronomy, Inc., for NASA, under contract NAS 5-26555. P.G. acknowledges NSF grants AST-0307966, AST-0607852, and AST-0507483. MG acknowledges support from NSF grant AST-0908752. C.S. acknowledges NSF grant AST-0909978. J.G.C. acknowledges NSF grant AST-090109. M.H.S. was supported at PSU by NASA contract NAS5-00136. This research used the facilities of the Canadian Astronomy Data Centre operated by the National
Research Council of Canada with the support of the Canadian Space Agency.

The authors wish to recognize and acknowledge the very significant cultural role and reverence that the summit of Mauna Kea has always had within the indigenous Hawaiian community. We are most fortunate to have the opportunity to conduct observations from this mountain.

Facility: Keck:II (DEIMOS)

\section{REFERENCES}

Adelman-McCarthy, J. K., et al. 2007, ApJS, 172, 634

An, D., et al. 2008, ApJS, 179, 326

Anders, E., \& Grevesse, N. 1989, Geochim. Cosmochim. Acta, 53, 197

Aoki, W., et al. 2009, A\&A, 502, 569

Armandroff, T. E., \& Da Costa, G. S. 1991, AJ, 101, 1329

Beers, T. C., Preston, G. W., \& Shectman, S. A. 1985, AJ, 90, 2089

Beers, T. C., Preston, G. W., \& Shectman, S. A. 1992, AJ, 103, 1987

Bell, E. F., et al. 2008, ApJ, 680, 295

Bell, R. A., Eriksson, K., Gustafsson, B., \& Nordlund, A. 1976, A\&AS, 23, 37

Bellazzini, M., Ferraro, F. R., Origlia, L., Pancino, E., Monaco, L., \& Oliva, E. 2002, AJ, 124, 3222

Bellazzini, M., Gennari, N., Ferraro, F. R., \& Sollima, A. 2004, MNRAS, 354, 708

Bellazzini, M., Pecci, F. F., Ferraro, F. R., Galleti, S., Catelan, M., \& Landsman, W. B. 2001, AJ, 122, 2569

Binney, J., \& Tremaine, S. 2008, Galactic Dynamics (2nd ed.; Princeton, NJ: Princeton Univ. Press)

Bond, H. E. 1980, ApJS, 44, 517

Brocato, E., Castellani, V., \& Ripepi, V. 1996, AJ, 111, 809

Buonanno, R., Pecci, F. F., Capellaro, E., Ortolani, S., Richtler, T., \& Geyer, E. H. 1991, AJ, 102, 1005

Carney, B. W., Storm, J., Trammell, S. R., \& Jones, R. V. 1992, PASP, 104, 44

Carretta, E., Gratton, R., Cohen, J. G., Beers, T. C., \& Christlieb, N. 2002, AJ, 124,481

Castelli, F. 2005, Mem. Soc. Astron. Ital. Suppl., 8, 34

Castelli, F., \& Kurucz, R. L. 2004, arXiv:astro-ph/0405087

Chonis, T. S., \& Gaskell, C. M. 2008, AJ, 135, 264

Clem, J. L., Vanden Berg, D. A., \& Stetson, P. B. 2008, AJ, 135, 682

Cohen, J. G., Christlieb, N., Beers, T. C., Gratton, R., \& Carretta, E. 2002, AJ, 124,470

Cohen, J. G., Christlieb, N., McWilliam, A., Shectman, S., Thompson, I., Melendez, J., Wisotzki, L., \& Reimers, D. 2008, ApJ, 672, 320

Cohen, J. G., \& Huang, W. 2009, ApJ, 701, 1053

Cohen, J. G., \& Huang, W. 2010, ApJ, 719, 931

Cohen, J. G., Kirby, E. N., Simon, J. D., \& Geha, M. C. 2010, ApJ, in press (arXiv:1010.0031)

Cohen, J. G., \& Meléndez, J. 2005a, AJ, 129, 303

Cohen, J. G., \& Meléndez, J. 2005b, AJ, 129, 1607

Cohen, J. G., et al. 2006, AJ, 132, 137

Cooper, A. P., et al. 2010, MNRAS, 406, 744

Côté, P., Richer, H. B., \& Fahlman, G. G. 1991, AJ, 102, 1358

Da Costa, G. S., Held, E. V., Saviane, I., \& Gullieuszik, M. 2009, ApJ, 705, 1481

Demarque, P., Woo, J.-H., Kim, Y.-C., \& Yi, S. K. 2004, ApJS, 155, 667

Durrell, P. R., \& Harris, W. E. 1993, AJ, 105, 1420

Eggen, O. J., Lynden-Bell, D., \& Sandage, A. R. 1962, ApJ, 136, 748

Faber, S. M., et al. 2003, Proc. SPIE, 4841, 1657

Ferraro, F. R., Clementini, G., Fusi Pecci, F., Sortino, R., \& Buonanno, R. 1992, MNRAS, 256, 391

Font, A. S., Johnston, K. V., Bullock, J. S., \& Robertson, B. E. 2006, ApJ, 638, 585

Frebel, A., Kirby, E., \& Simon, J. D. 2010a, Nature, 464, 72

Frebel, A., Simon, J. D., Geha, M., \& Willman, B. 2010b, ApJ, 708, 560

Freeman, K. C., \& Rodgers, A. W. 1975, ApJ, 201, L71

Fulbright, J. P. 2000, AJ, 120, 1841

Fulbright, J. P., Rich, R. M., \& Castro, S. 2004, ApJ, 612, 447

Geffert, M., \& Maintz, G. 2000, A\&AS, 144, 227

Geisler, D., Smith, V. V., Wallerstein, G., Gonzalez, G., \& Charbonnel, C. 2005, AJ, 129, 1428

Girardi, L., Bertelli, G., Bressan, A., Chiosi, C., Groenewegen, M. A. T., Marigo, P., Salasnich, B., \& Weiss, A. 2002, A\&A, 391, 195

Gratton, R., Sneden, C., \& Carretta, E. 2004, ARA\&A, 42, 385

Gratton, R. G. 1982, ApJ, 257, 640 
Gratton, R. G., Carretta, E., Desidera, S., Lucatello, S., Mazzei, P., \& Barbieri, M. 2003, A\&A, 406, 131

Gratton, R. G., \& Ortolani, S. 1989, A\&A, 211, 41

Gray, D. F. 2008, The Observation and Analysis of Stellar Photospheres (3rd ed.; Cambridge: Cambridge Univ Press)

Guhathakurta, P., et al. 2006, AJ, 131, 2497

Gustafsson, B., Bell, R. A., Eriksson, K., \& Nordlund, A. 1975, A\&A, 42, 407

Gustafsson, B., Edvardsson, B., Eriksson, K., Jørgensen, U. G., MizunoWiedner, M., \& Plez, B. 2003, in ASP Conf. Ser. 288, Modelling of Stellar Atmospheres, ed. I. Hubeny, D. Mihalas, \& K. Werner (San Francisco, CA: ASP), 331

Gustafsson, B., Edvardsson, B., Eriksson, K., Jørgensen, U. G., Nordlund, Å., \& Plez, B. 2008, A\&A, 486, 951

Harris, W. E. 1975, ApJS, 29, 397

Harris, W. E. 1996, AJ, 112, 1487

Harris, W. E., et al. 1997, AJ, 114, 1030

Irwin, M., \& Hatzidimitriou, D. 1995, MNRAS, 277, 1354

Ivans, I. I., Kraft, R. P., Sneden, C., Smith, G. H., Rich, R. M., \& Shetrone, M. 2001, AJ, 122, 1438

Johnson, J. A. 2002, ApJS, 139, 219

Jordi, K., Grebel, E. K., \& Ammon, K. 2006, A\&A, 460, 339

Kirby, E. N., Cohen, J. G., Smith, G. H., Majewski, S. R., Sohn, S. T., \& Guhathakurta, P. 2010a, ApJ, in press (arXiv:1011.5221) (Paper IV)

Kirby, E. N., Guhathakurta, P., Bolte, M., Sneden, C., \& Geha, M. C. 2009, ApJ, 705, 328 (Paper I)

Kirby, E. N., Guhathakurta, P., \& Sneden, C. 2008a, ApJ, 682, 1217 (KGS08)

Kirby, E. N., Lanfranchi, G. A., Simon, J. D., Cohen, J. G., \& Guhathakurta, P. 2010b, ApJ, in press (arXiv:1011.4937) (Paper III)

Kirby, E. N., Simon, J. D., Geha, M., Guhathakurta, P., \& Frebel, A. 2008b, ApJ, $685, \mathrm{~L} 43$

Koch, A., Grebel, E. K., Gilmore, G. F., Wyse, R. F. G., Kleyna, J. T., Harbeck, D. R., Wilkinson, M. I., \& Wyn Evans, N. 2008, AJ, 135, 1580

Kravtsov, V., Ipatov, A., Samus, N., Smirnov, O., Alcaino, G., Liller, W., \& Alvarado, F. 1997, A\&AS, 125, 1

Kuehn, C., et al. 2008, ApJ, 674, L81

Kupka, F., Piskunov, N., Ryabchikova, T. A., Stempels, H. C., \& Weiss, W. W. 1999, A\&AS, 138, 119

Kurucz, R. 1993, ATLAS9 Stellar Atmosphere Programs and 2 km/s grid, Kurucz CD-ROM No. 13 (Cambridge, MA.: Smithsonian Astrophysical Observatory), 13

Kurucz, R. 2005, Mem. Soc. Astron. Ital. Suppl., 8, 14

Lai, D. K., Bolte, M., Johnson, J. A., \& Lucatello, S. 2004, AJ, 128, 2402

Lai, D. K., Johnson, J. A., Bolte, M., \& Lucatello, S. 2007, ApJ, 667, 1185

Lee, M. G., et al. 2003, AJ, 126, 2840

Letarte, B., et al. 2010, A\&A, 523, A17

Majewski, S. R. 1993, ARA\&A, 31, 575

Majewski, S. R., Munn, J. A., \& Hawley, S. L. 1996, ApJ, 459, L73

Majewski, S. R., Ostheimer, J. C., Kunkel, W. E., \& Patterson, R. J. 2000, AJ, 120,2550

Marino, A. F., Milone, A. P., Piotto, G., Villanova, S., Bedin, L. R., Bellini, A., \& Renzini, A. 2009, A\&A, 505, 1099

Markwardt, C. B. 2009, in ASP Conf. Ser. 411, Astronomical Data Analysis Software and Systems XVIII, ed. D. A. Bohlender, D. Durand, \& P. Dowler (San Francisco CA: ASP), 251

Martin, N. F., de Jong, J. T. A., \& Rix, H.-W. 2008, ApJ, 684, 1075

Mateo, M. L. 1998, ARA\&A, 36, 435

Mighell, K. J., \& Burke, C. J. 1999, AJ, 118, 366

Mishenina, T. V., Panchuk, V. E., \& Samus', N. N. 2003, Astron.. Rep., 47, 248

Norris, J. E., Ryan, S. G., \& Beers, T. C. 1999, ApJS, 123, 639

Paltrinieri, B., Ferraro, F. R., Carretta, E., \& Fusi Pecci, F. 1998, MNRAS, 293, 434

Pietrzyński, G., et al. 2008, AJ, 135, 1993
Pilachowski, C. A., Sneden, C., \& Kraft, R. P. 1996, AJ, 111, 1689

Piskunov, A. E., Schilbach, E., Kharchenko, N. V., Röser, S., \& Scholz, R.-D. 2007, A\&A, 468, 151

Pritzl, B. J., Venn, K. A., \& Irwin, M. 2005, AJ, 130, 2140

Ramírez, S. V., \& Cohen, J. G. 2002, AJ, 123, 3277

Ramírez, S. V., \& Cohen, J. G. 2003, AJ, 125, 224

Reed, B. C., Hesser, J. E., \& Shawl, S. J. 1988, PASP, 100, 545

Regnault, N., et al. 2009, A\&A, 506, 999

Rizzi, L., Held, E. V., Saviane, I., Tully, R. B., \& Gullieuszik, M. 2007, MNRAS, 380, 1255

Robertson, B., Bullock, J. S., Font, A. S., Johnston, K. V., \& Hernquist, L. 2005, ApJ, 632, 872

Roederer, I. U. 2009, AJ, 137, 272

Rosenberg, A., Piotto, G., Saviane, I., \& Aparicio, A. 2000, A\&AS, 144, 5

Rutledge, G. A., Hesser, J. E., \& Stetson, P. B. 1997, PASP, 109, 907

Sadakane, K., Arimoto, N., Ikuta, C., Aoki, W., Jablonka, P., \& Tajitsu, A. 2004, PASJ, 56, 1041

Sandquist, E. L., Bolte, M., Stetson, P. B., \& Hesser, J. E. 1996, ApJ, 470, 910

Sbordone, L. 2005, Mem. Soc. Astron. Ital. Suppl., 8, 61

Sbordone, L., Bonifacio, P., Castelli, F., \& Kurucz, R. L. 2004, Mem. Soc. Astron. Ital. Suppl., 5, 93

Schlegel, D. J., Finkbeiner, D. P., \& Davis, M. 1998, ApJ, 500, 525

Searle, L., \& Zinn, R. 1978, ApJ, 225, 357

Ségall, M., Ibata, R. A., Irwin, M. J., Martin, N. F., \& Chapman, S. 2007, MNRAS, 375, 831

Shetrone, M. D., Bolte, M., \& Stetson, P. B. 1998, AJ, 115, 1888

Shetrone, M. D., Côté, P., \& Sargent, W. L. W. 2001, ApJ, 548, 592

Shetrone, M. D., Siegel, M. H., Cook, D. O., \& Bosler, T. 2009, AJ, 137, 62

Shetrone, M. D., Venn, K. A., Tolstoy, E., Primas, F., Hill, V., \& Kaufer, A. 2003, AJ, 125, 684

Siegel, M. H., Majewski, S. R., Cudworth, K. M., \& Takamiya, M. 2001, AJ, 121,935

Siegel, M. H., Majewski, S. R., Sohn, S. T., Shetrone, M. D., Munoz, R. R., \& Patterson, R. J. 2010, ApJ, submitted

Simon, J. D., Frebel, A., McWilliam, A., Kirby, E. N., \& Thompson, I. B. 2010, ApJ, 716, 446

Simon, J. D., \& Geha, M. 2007, ApJ, 670, 313

Sohn, S. T., et al. 2007, ApJ, 663, 960

Sneden, C. A. 1973, PhD thesis, Univ. Texas at Austin

Sneden, C., Kraft, R. P., Guhathakurta, P., Peterson, R. C., \& Fulbright, J. P. 2004, AJ, 127, 2162

Sneden, C., Kraft, R. P., Prosser, C. F., \& Langer, G. E. 1992, AJ, 104, 2121

Sneden, C., Kraft, R. P., Shetrone, M. D., Smith, G. H., Langer, G. E., \& Prosser, C. F. 1997, AJ, 114, 1964

Sneden, C., Pilachowski, C. A., \& Kraft, R. P. 2000, AJ, 120, 1351

Spite, M. 1967, Ann. Astrophys., 30, 211

Stetson, P. B. 2000, PASP, 112, 925

Stetson, P. B., Hesser, J. E., \& Smecker-Hane, T. A. 1998, PASP, 110, 533

Thévenin, F., \& Idiart, T. P. 1999, ApJ, 521, 753

Tolstoy, E., Irwin, M. J., Cole, A. A., Pasquini, L., Gilmozzi, R., \& Gallagher, J. S. 2001, MNRAS, 327, 918

Tucker, D. L., et al. 2006, Astron. Nachr., 327, 821

Venn, K. A., Irwin, M., Shetrone, M. D., Tout, C. A., Hill, V., \& Tolstoy, E. 2004, AJ, 128, 1177

Webbink, R. F. 1985, in IAU Symp. 113, Dynamics of Star Clusters, ed. J. Goodman \& P. Hut (Dordrecht: Reidel), 541

Westfall, K. B., Majewski, S. R., Ostheimer, J. C., Frinchaboy, P. M., Kunkel, W. E., Patterson, R. J., \& Link, R. 2006, AJ, 131, 375

White, S. D. M., \& Rees, M. J. 1978, MNRAS, 183, 341

Zacharias, N., Monet, D. G., Levine, S. E., Urban, S. E., Gaume, R., \& Wycoff, G. L. 2004, BASS, 36, 1418

Zinn, R. 1985, ApJ, 293, 424

Zucker, D. B., et al. 2006, ApJ, 643, L103 